\title{
National Ignition Facility Pollution Prevention and Waste Minimization Plan
}

September 1, 1998

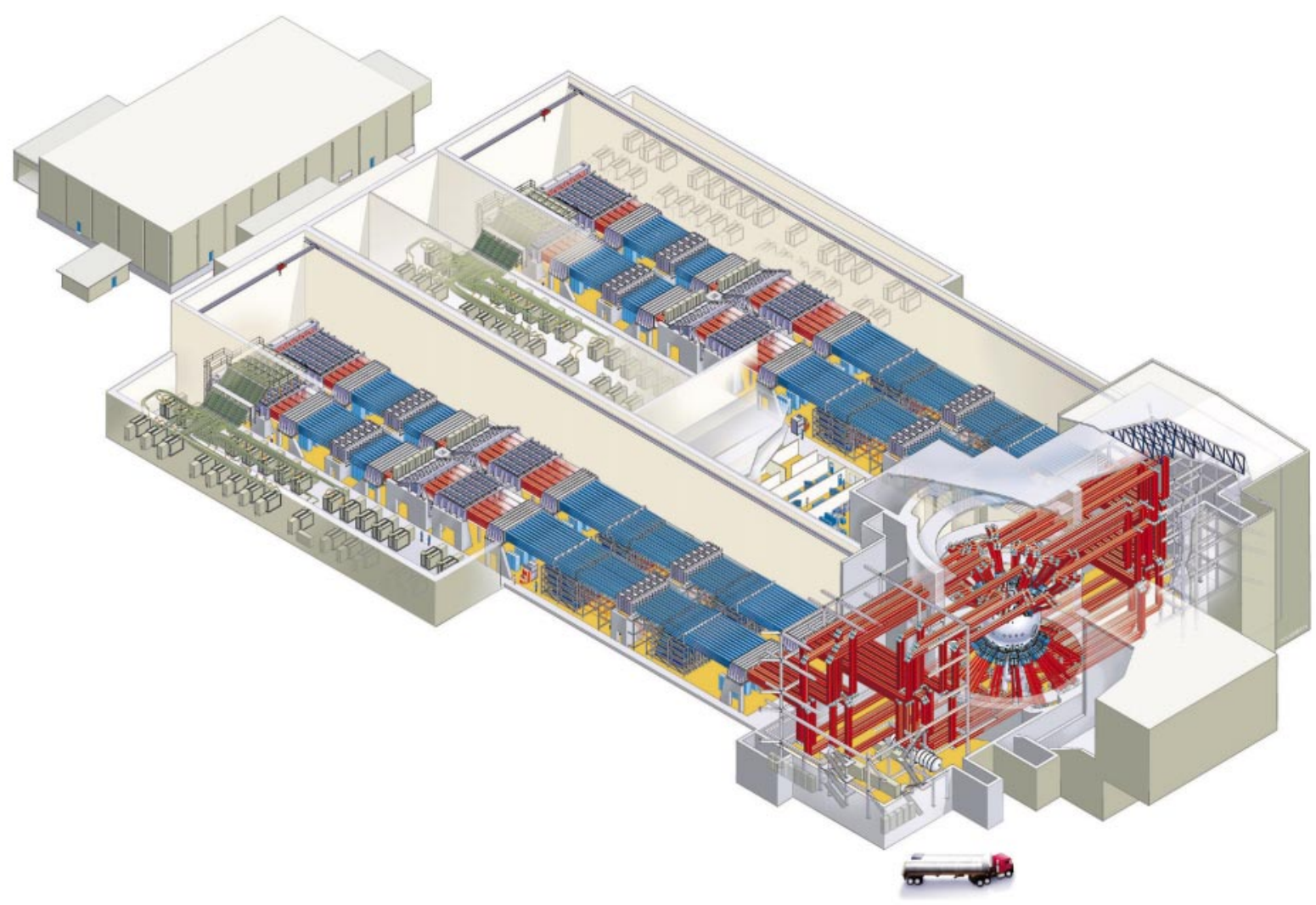


DISCLAIMER

This document was prepared as an account of work sponsored by an agency of the United States Government. Neither the United States Government nor the University of California nor any of their employees, makes any warranty, express or implied, or assumes any legal liability or responsibility for the accuracy, completeness, or usefulness of any information, apparatus, product, or process disclosed, or represents that its use would not infringe privately owned rights. Reference herein to any specific commercial products, process, or service by trade name, trademark, manufacturer, or otherwise, does not necessarily constitute or imply its endorsement, recommendation, or favoring by the United States Government or the University of California. The views and opinions of authors expressed herein do not necessarily state or reflect those of the United States Government or the University of California, and shall not be used for advertising or product endorsement purposes.

This report has been reproduced

directly from the best available copy.

Available to DOE and DOE contractors from the

Office of Scientific and Technical Information

P.O. Box 62, Oak Ridge, TN 37831

Prices available from (615) 576-8401, FTS 626-8401

Available to the public from the

National Technical Information Service

U.S. Department of Commerce

5285 Port Royal Rd.,

Springfield, VA 22161

Work performed under the auspices of the U.S. Department of Energy by Lawrence Livermore National Laboratory under Contract W-7405-Eng-48. 


\title{
National Ignition Facility \\ Pollution Prevention and \\ Waste Minimization Plan
}

\author{
September 1, 1998
}

\section{Lawrence Livermore National Laboratory}

University of California • Livermore, California • 94550 



\title{
National Ignition Facility Pollution Prevention and Waste Minimization Plan
}

\author{
September 1, 1998
}

Responsible individual:

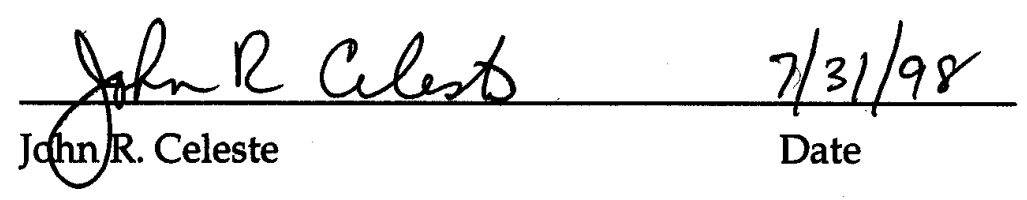

Pollution Prevention Group Leader

Reviewed and accepted by:

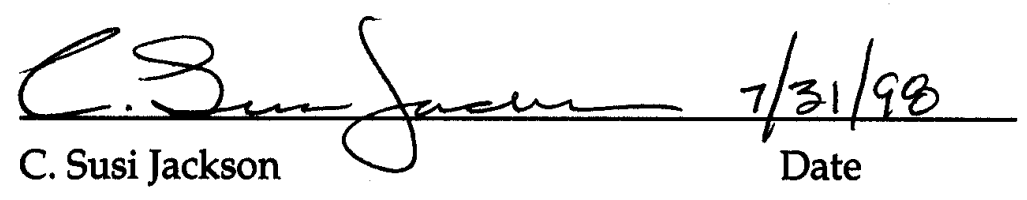

Operations and Regulatory Affairs Division Leader

Approved by:

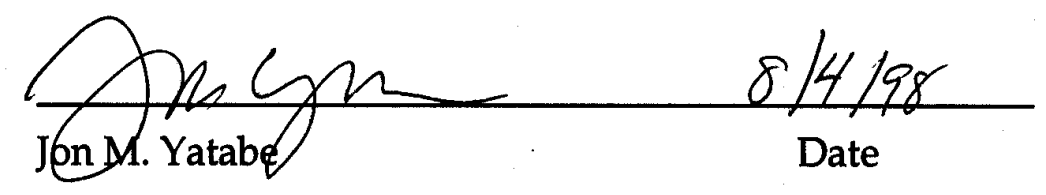

Assurance Manager, NIF

Approved by:

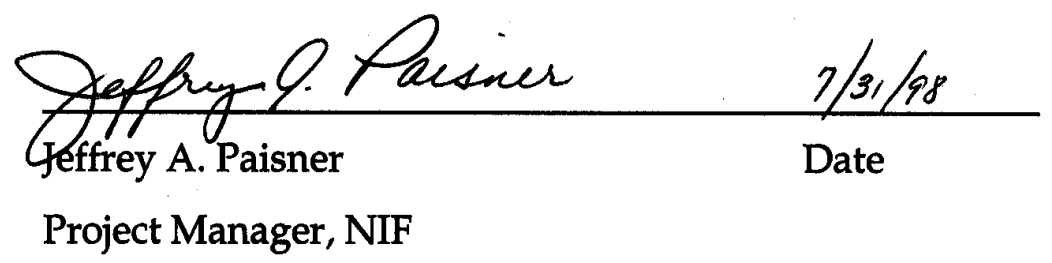

Concurrence by:

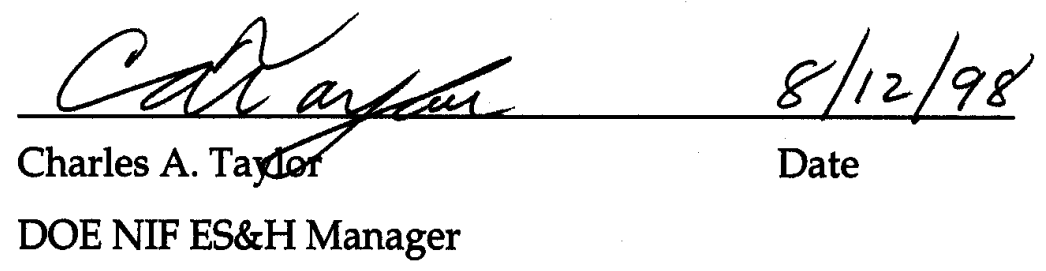


NIF-0010575

UCRL-AR-131194 


\section{Contents}

Abbreviations, Acronyms, and Definitions.................................................... vi

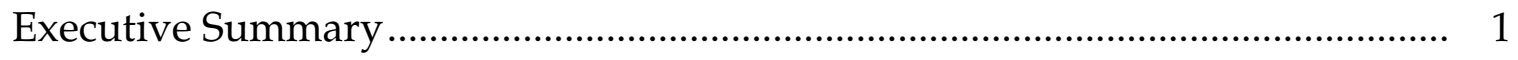

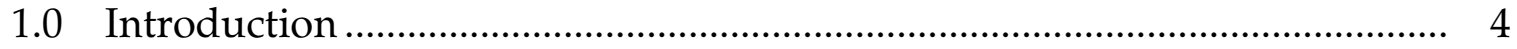

1.1 Overview of the National Ignition Facility ....................................... 4

1.2 Pollution Prevention and Waste Minimization ................................ 6

1.3 Purpose of the NIF P2/WMin Plan ................................................ 6

2.0 Scope: Roles, Responsibilities, and Goals................................................ 7

2.1 Environmental Protection ................................................................ 8

2.2 P2 Roles and Responsibilities ........................................................ 8

2.3 Regulatory Compliance..................................................................... 9

2.4 NIF P2/WMin Goals....................................................................... 12

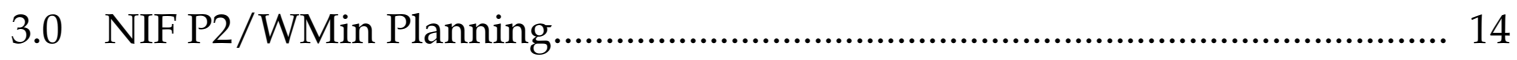

3.1 Design for Environment (DfE) Planning .......................................... 14

3.2 Construction-Phase Planning ............................................................ 15

3.3 Activation-Phase and Operational-Phase Planning............................ 15

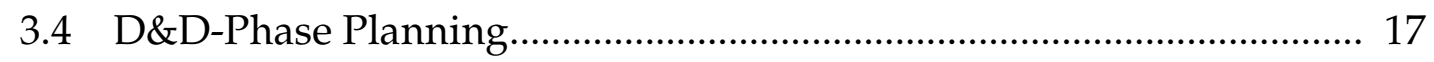

4.0 Implementation of Waste Stream Monitoring ........................................... 19

4.1 Waste Stream Monitoring and Management..................................... 19

4.2 Waste Stream Monitoring and Reuse............................................... 19

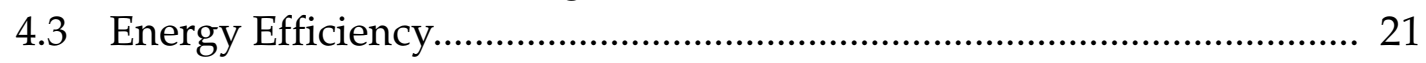

4.4 Environmental Costs .................................................................... 24

4.5 Reuse and Recycling Methods ........................................................ 24

4.6 NIF P2/WMin Review and Methods ................................................ 27

5.0 Predictive PPOAs for NIF Waste Streams ............................................... 31

5.1 PPOA 1: Vacuum Pump Oil .......................................................... 31

5.2 PPOA 2: Molecular Sieves............................................................... 33

5.3 PPOA 3: Personal Protective Equipment........................................... 38

5.4 PPOA 4A: Pre- and HEPA Filters .................................................... 39

5.5 PPOA 4B: Target Chamber Decontamination...................................... 42

5.6 PPOA 5: Target Chamber Hardware.................................................. 46

5.7 PPOA 6: Debris Shield Decontamination ........................................ 52

5.8 PPOA 7: Capacitors, Oil-Filled....................................................... 56

5.9 PPOA 8A: General Chemicals .............................................................. 58 
NIF-0010575

UCRL-AR-131194

5.10 PPOA 8B: General Chemicals, Optical Component Processing........... 65

6.0 Accomplishments to Date............................................................................ 72

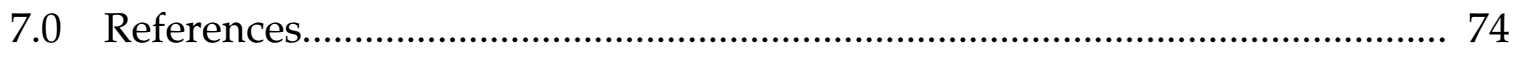

Appendix A. Method of Preparing a PPOA.......................................................... 76

\section{Figures}

Figure 1-1. Layout of the LLNL site showing location of the NIF ........................ 5

Figure 2-1. Simplified NIF timeline ................................................................. 7

Figure 4-1. Major NIF construction materials for recycling................................... 25

Figure PPOA 1.1. Vacuum system schematic .......................................................... 33

Figure PPOA 2.1. Tritium processing system layout............................................... 35

Figure PPOA 2.2. Tritium processing system module ............................................ 36

Figure PPOA 4B.1. Louvered first-wall panel............................................................ 43

Figure PPOA 4B.2. Louvered first-wall panel (edge view)...................................... 43

Figure PPOA 5.1. Target positioner from target insertion side.............................. 47

Figure PPOA 6.1. Final optics assembly showing location of debris shield......... 53

Figure PPOA 6.2. Debris shield decontamination process....................................... 54

Figure PPOA 7.1. Beamlet capacitor bank module .................................................. 57

Figure PPOA 8A.1. OAB mechanical parts cleaning flow ........................................ 60 


\section{Tables}

Table 4-1. LTAB energy-conservation alternatives assessed .............................. 22

Table I.4.1.2.8.1-2. National Ignition Facility waste estimates ............................. 30

Table PPOA 5.1. Phase I diagnostics ............................................................ 49

Table PPOA 5.2. Phase II diagnostics............................................................ 49

Table PPOA 6.1. LLNL internal discharge limits ............................................ 55

Table PPOA 8A.1. MIL-STD-1246C Level 50A: NVR cleanliness level................ 60

Table PPOA 8A.2. MIL-STD-1246C Level 50A: particle cleanliness level ........... 60

Table PPOA 8A.3. MIL-STD-1246C Level 100A and 500 cleanliness level.......... 62

Table 6.1. P2/WMin accomplishments ........................................................... 73 


\section{Abbreviations, Acronyms, and Definitions}

AB 939

$\mathrm{AD}$

APCD

AQMD

AR

AVLIS

BAAQMD

CAAQS

CCAA

CCD

CCR

CDR

CFR

ChemTrack

CHEW

D\&D

DEHP

DfE

DIM

DOE

DOE/OAK

DTSC

DUS

EPA
California State Assembly Bill 939 enacted in 1989 requiring solidwaste-stream reduction by cities and counties

Associate Director of LLNL

Air Pollution Control District

Air Quality Management District

antireflective

LLNL Atomic Vapor Laser Isotope Separation Program

Bay Area Air Quality Management District

California Ambient Air Quality Standards

California Clean Air Act

charged-coupled device

California Code of Regulations

National Ignition Facility Conceptual Design Report (NIF-LLNL-94113 and UCRL-PROP-117903)

Code of Federal Regulations

LLNL inventory system that monitors the acquisition, storage, distribution, and use of chemicals by LLNL programs and projects

Chemical Exchange Warehouse at LLNL-assists programs in using reclaimed, recycled, or previously opened chemical materials

decontamination and decommissioning activities to be conducted at the end of the NIF life cycle

diethylhexylphthlalate-the dielectric fluid used in Nova electrical capacitors, but not to be used in the NIF electrical capacitors

Design for Environment-DfE planning incorporates environmental considerations into the design or redesign of products, processes, projects, and technical or management systems

diagnostic instrument manipulator-part of the NIF target chamber hardware package

\section{United States Department of Energy}

United States Department of Energy, Oakland Operations Office

California State Department of Toxic Substances Control

Donation, Utilization, and Sales Group at LLNL—materials forwarded to DUS for reuse are assigned to other LLNL programs, donated to government agencies and local school districts, or sold to a reclamation vendor

United States Environmental Protection Agency 


\begin{tabular}{|c|c|}
\hline EPCRA & $\begin{array}{l}\text { Federal Emergency Planning and Community Right-to-Know Act, } \\
\text { Section } 313\end{array}$ \\
\hline EPD & Environmental Protection Department at LLNL \\
\hline ES\&H & Environmental Safety and Health \\
\hline ETEC & Energy Technology Engineering Center \\
\hline FOA & $\begin{array}{l}\text { Final Optics Assembly-optical subsystems inserted into the NIF } \\
\text { target chamber }\end{array}$ \\
\hline HEPA & $\begin{array}{l}\text { high-efficiency particulate air (filter)—removes particulate matter from } \\
\text { buildings and systems at various stipulated efficiencies }\end{array}$ \\
\hline HVAC & heating, ventilating, and air-conditioning systems \\
\hline HWMD & Hazardous Waste Management Division at LLNL \\
\hline ICF & $\begin{array}{l}\text { inertial confinement fusion-the process of joining light nuclei using a } \\
\text { driver that delivers an intense pulse of energy to a target containing } \\
\text { fusion fuel; the delivered energy causes the fuel capsule to implode, } \\
\text { creating the high densities and temperatures necessary for the fusion } \\
\text { reaction to occur }\end{array}$ \\
\hline $\mathrm{KD} * \mathrm{P}$ & $\begin{array}{l}\text { deuterated analog of the potassium dihydrogen phosphate crystals } \\
\text { used in NIF optical arrays }\end{array}$ \\
\hline KDP & potassium dihydrogen phosphate crystals used in NIF optical arrays \\
\hline LLNL & $\begin{array}{l}\text { Lawrence Livermore National Laboratory and its remote experimental } \\
\text { test base, Site 300, which is located in rural Alameda and San Joaquin } \\
\text { counties, near Tracy, CA }\end{array}$ \\
\hline LLW & low-level waste \\
\hline LRU & line replaceable unit-laser subsystems used by the NIF \\
\hline LTAB & Laser and Target Area Building of the NIF \\
\hline LWRP & Livermore Water Reclamation Plant \\
\hline MAP & $\begin{array}{l}\text { Mitigation Action Plan required by DOE Order } 451.1 \text {, a document } \\
\text { describing mitigation measures to address the environmental impacts } \\
\text { presented in the Record of Decision }\end{array}$ \\
\hline NAAQS & National Ambient Air Quality Standards \\
\hline NESHAP & National Emission Standards for Hazardous Air Pollutants \\
\hline NIF & $\begin{array}{l}\text { National Ignition Facility-used in this document to mean the NIF } \\
\text { project or the research/experimental complex, depending on the } \\
\text { context }\end{array}$ \\
\hline NIF E\&S & NIF-specific environmental and safety working group \\
\hline Nova & $\begin{array}{l}\text { solid-state, neodymium-glass, ten-beam laser-the primary ICF } \\
\text { research tool at LLNL from } 1985 \text { to } 1999\end{array}$ \\
\hline NPDES & National Pollutant Discharge Elimination System \\
\hline NVR & nonvolatile residue-a measure of the cleanliness level achieved \\
\hline $\mathrm{OAB}$ & Optics Assembly Building of the NIF \\
\hline
\end{tabular}


OPG optical pulse generating system - the NIF "front-end" system that generates, shapes, and smoothes laser energy

ORAD Operations and Regulatory Affairs Division of LLNL, within the Environmental Protection Department

P2 abbreviation for pollution prevention-strategies and principles that reduce, reuse, and recycle waste

P2/WMin pollution prevention and waste minimization

P2/Wmin Plan the shorthand name of this document

PEIS Final Programmatic Environmental Impact Statement for Stockpile Stewardship and Management (DOE/EIS-0236, September 1996)

PPE personal protective equipment-used by workers to shield them from toxic or radioactive materials

PPG Pollution Prevention Group-within the Environmental Protection Department, Operations and Regulatory Affairs Division, LLNL

PPOA Pollution Prevention Opportunity Assessment-a program-specific P2 evaluation provided by PPG personnel

PSAR National Ignition Facility Preliminary Safety Analysis Report (NIFLLNL-96-238 and UCRL-ID-123759)

RCRA Resource Conservation and Recovery Act-federal legislation requiring a hazardous waste generator to establish a program that reduces the volume and toxicity of its wastes to the greatest degree "economically practicable"

ROI return on investment-financial calculation used to evaluate the economic benefits of pollution prevention alternatives

SAER

SB 14 LLNL Site Annual Environmental Report (UCRL-50027-96, 1996 edition)

SPCC

California Senate Bill 14-the Hazardous Waste Source Reduction and Management Review Act of 1989

Spill Prevention Control and Countermeasures—a spill-prevention plan at LLNL

SWPPP Storm Water Pollution Prevention Plan

target

TAS the item containing fusion fuel on which the NIF laser beams are focused: direct-drive targets consist of a fuel capsule; indirect-drive targets consist of a fuel capsule surrounded by a hollow metal cylinder target alignment sensor-part of the target-positioning hardware within the NIF target chamber

TASPOS target alignment sensor positioner-a manipulator that allows remote positioning of the target alignment sensor within the NIF target chamber

TEOS tetraethyl orthosilicate-a chemical constituent of the antireflective coatings applied to NIF optics

TMOS methyl triethloxy silane 
TPS

TQM

WBS

WMin
Tritium Processing System of the NIF

Total Quality Method—used to evaluate and assess pollution prevention strategies

work breakdown structure-used by NIF project management to track various elements of the facility

abbreviation for waste minimization-actions that reduce the generation of waste, reduce the toxicity of waste, improve energy efficiency, or maximize recycling efforts 



\section{Executive Summary}

Pollution prevention (P2) is a systematic assessment of materials, processes, or practices that eliminates or reduces the release of pollutants, contaminants, hazardous substances, and waste into the land, air, or water. P2 attempts to eliminate waste generation before it occurs. If such elimination is not possible, then waste minimization (WMin) - the minimization of waste streams once they are being generated-represents the next level in a waste management hierarchy. Recycling, the next-preferred approach, involves the reuse or regeneration of materials and wastes into usable products. Treatment and disposal are the last-resort measures.

P2 analysis is routinely performed on the waste streams from operating facilities; however, such analysis can be significantly more effective when it is done during the design phase of a facility. P2 includes practices that eliminate or minimize the discharge of all wastes, including hazardous, radioactive, or toxic materials, to the environment.

This document is the Lawrence Livermore National Laboratory (LLNL) National Ignition Facility (NIF) Pollution Prevention and Waste Minimization Plan. It will not only function as the planning document for anticipating, minimizing, and mitigating NIF waste generation, but it is also a Department of Energy (DOE) milestone document specified in the facility's Mitigation Action Plan (MAP). As such, it is one of the "living" reference documents that will guide NIF operations through all phases of the project. This document will be updated periodically to reflect development of the NIF, from construction through lifetime operation (see the NIF timeline, below).

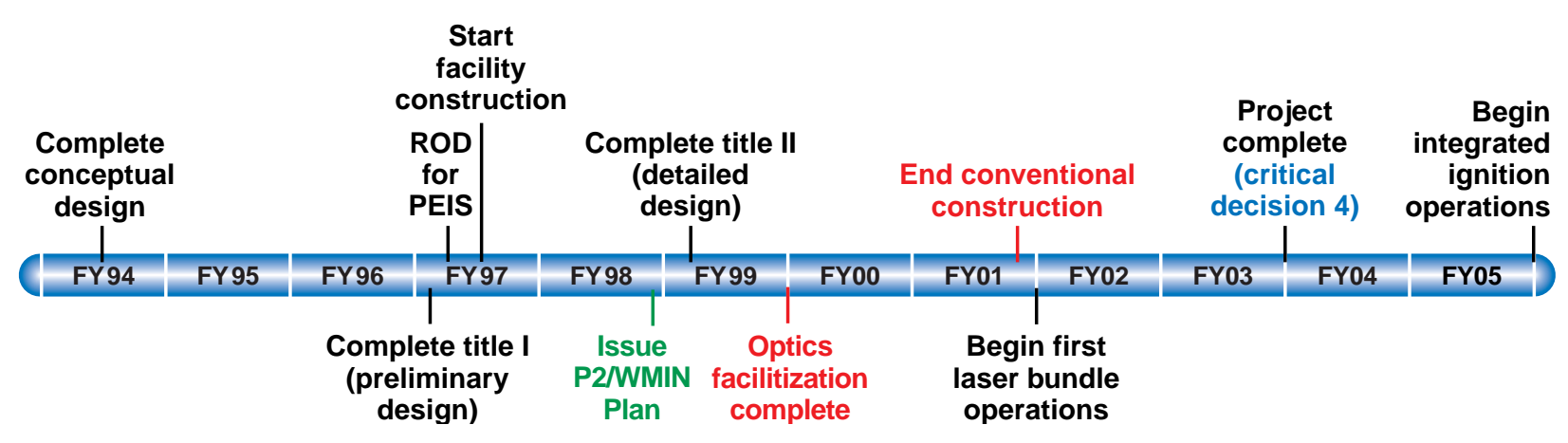

NIF timeline.

The NIF project team and researchers from the Pollution Prevention Group (PPG), a group within the LLNL Environmental Protection Department, assembled this plan as a joint effort. During the investigations undertaken for the document, NIF project personnel demonstrated a genuine commitment to minimize the environmental impact of the project to the full extent technically feasible and economically possible. 
WMin activities benefiting the construction phase of the NIF are discussed in this document. Energy consumption anticipated for the operational phase of the NIF has been analyzed, and the results are briefly summarized.

This document also evaluates the NIF design from a pollution prevention perspective. Nonhazardous NIF wastes will not differ substantially from those already occurring at LLNL. Hazardous liquid wastes will be the product of normal cleaning and maintenance operations, and they will consist primarily of solvents, acids, paints, and oils. Hazardous solid wastes will consist primarily of hardware that has failed or is beyond its useful life, with no possibility of recycling or reuse. Low-level radioactive wastes will originate in the target chamber and from any cleaning activities associated with components or insertions into the target chamber. Nonhazardous water-based cleaners used to decontaminate surfaces, molecular sieve media, wipes, and personal protective equipment (PPE) from decontamination operations and high-efficiency particulate air (HEPA) filters will make up the low-level waste (LLW) stream. Mixed waste streams will be generated primarily by decontamination operations using solvents that become metal-contaminated and by debris-shield cleaning operations.

The primary waste streams identified in the Final Programmatic Environmental Impact Statement for Stockpile Stewardship and Management (PEIS), issued by the DOE (DOE/EIS0236, September 1996) are evaluated in this document in predictive pollution prevention opportunity assessments (PPOAs). The predictive PPOAs discussed in Section 5.0 of this document are as follows:

1. Vacuum pump oil.

2. Molecular sieves.

3. Personal protective equipment.

4.A. Pre- and HEPA filters.

4.B. Target chamber decontamination.

5. Hardware from the chamber.

6. Debris shield cleaning.

7. Capacitors, oil-filled.

8.A. General chemicals.

8.B. General chemicals, optical component processing, B391.

This document also outlines a P2 plan for an operational NIF. The NIF will commence integrated ignition operations in approximately 10/05. The scope of the P2 plan for the operational NIF is the same as that of the LLNL site-wide Pollution Prevention Plan (UCRL-AR-127073).

Section 6.0 of this document summarizes the significant NIF P2/WMin accomplishments that have taken place as of the publication date (September 1998). The accomplishments include the development of low-activation concrete formulations for the target area, substitution of aqueous cleaning lines for many solvent-cleaning processes, integration of P2 in the design of processes throughout the work breakdown structure (WBS), and testing and substitution of oil-free vacuum pumps throughout the 
facility. The most significant recycling accomplishment to date has been the reuse of more than 281,000 metric tons of soil excavated during construction, as shown in the illustration below. To date, the NIF P2/WMin activities have saved costs, reduced volumes of waste streams, and eliminated some expected waste streams. Additional accomplishments are expected as the PPOAs are implemented, and they will be documented as this plan is updated.

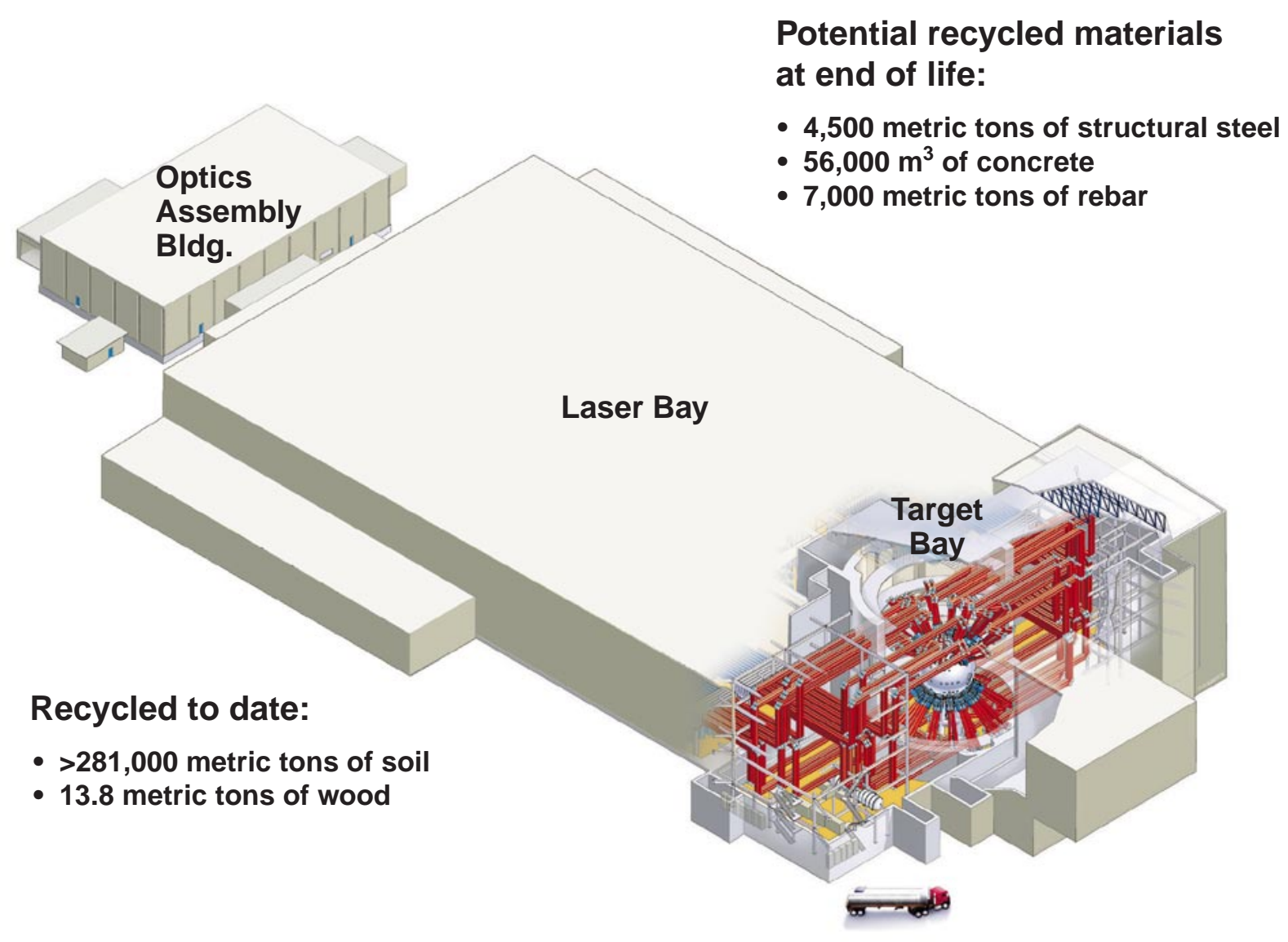

NIF construction materials recycling. 


\subsection{Introduction}

\subsection{Overview of the National Ignition Facility}

The National Ignition Facility (NIF) is a U.S. Department of Energy (DOE) inertial confinement fusion (ICF) laser facility now under construction at Lawrence Livermore National Laboratory (LLNL). As shown in Figure 1-1, the NIF is located in the northeastern quadrant of LLNL.

The NIF mission is to achieve ICF ignition, to assess physical conditions in matters of interest to nuclear weapons physics, to provide an above-ground simulation capability for nuclear-weapons-effects testing, to contribute to the development of inertial fusion for electrical power generation, and to support basic science and technology. To achieve its mission, the facility will require a laser with an output pulse energy of $1.8 \mathrm{MJ}$ and an output pulse power of 500 TW. The laser will consist of 192 identical beamlets. Each beam of light will be focused onto a target suspended in the center of the spherical aluminum-alloy target chamber. The target chamber will be housed inside a cylindrical, reinforced-concrete target bay with 1.8-m-thick walls for radiation shielding. The target bay will be located within the Laser and Target Area Building (LTAB), which is the main experimental building of the NIF. The LTAB will consist of two laser bays, four capacitor bays, two optical switchyards, a target bay, an attached diagnostics building, a decontamination area, and operational support areas.

NIF construction began in mid-1997 and is ongoing. The Title II phase of special equipment design (final design) and procurement are underway. When it is complete, the NIF will be composed of five primary elements:

- Conventional facilities-the Laser and Target Area Building (LTAB) that houses the laser and target systems, and the Optics Assembly Building (OAB) that provides refurbishment capability for optical components.

- Laser systems-192 laser beams that generate and deliver high-power, shortduration optical pulses to the target chamber. Multiple beams will be configured to uniformly irradiate the target.

- Target systems-all the components used to perform target experiments, such as the target chamber, target positioner, target diagnostics, and environmental protection systems.

- Integrated computer control systems-the network of computers providing the hardware and software necessary to operate, control (e.g., interlock access control, etc.), and obtain data from the NIF.

- Optical components-the many light-pulse-shaping arrays required for specific research and test experiments. 


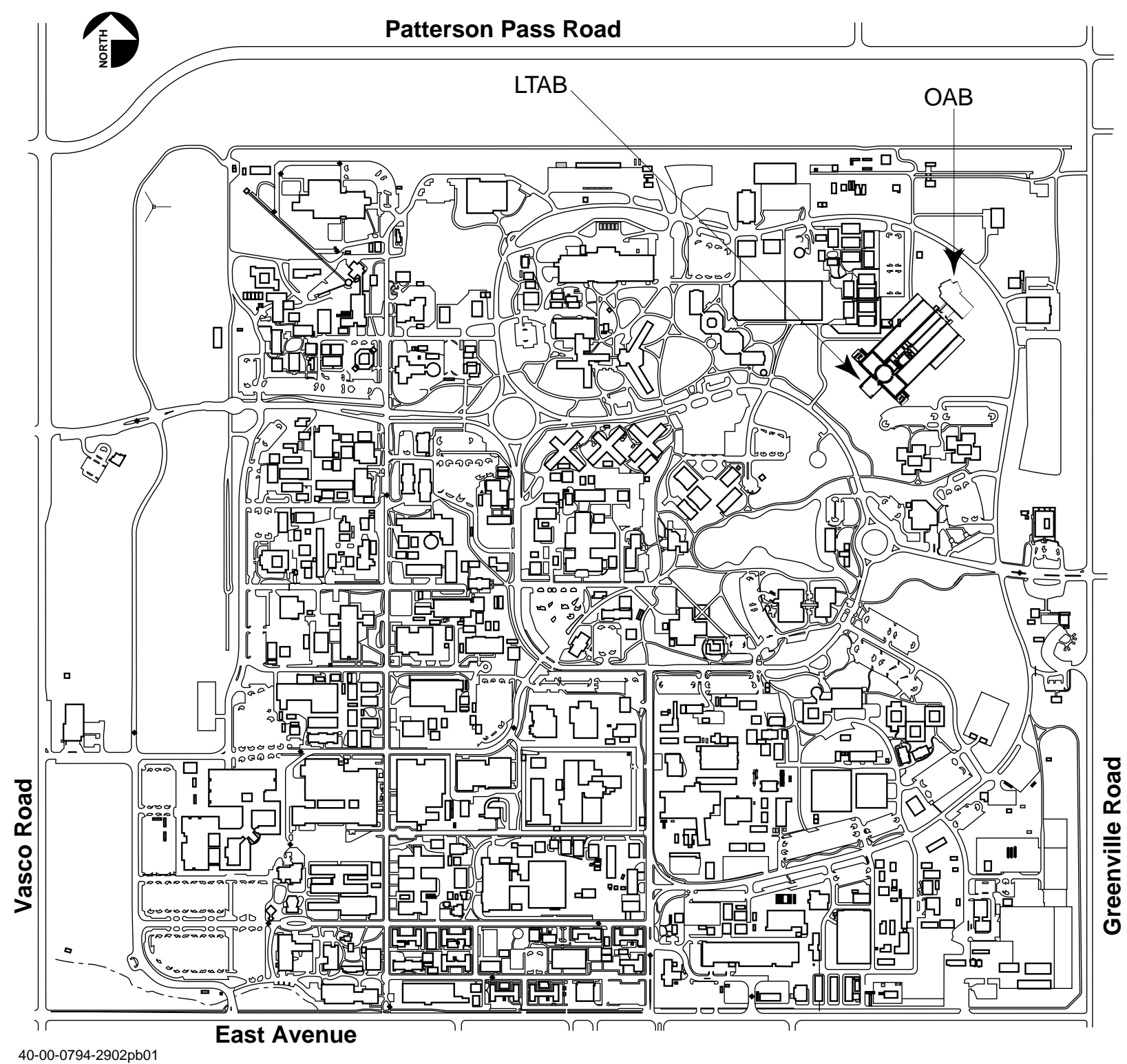

Figure 1-1. Layout of the LLNL site showing the location of the NIF Laser and Target Area Building (LTAB) and Optics Assembly Building (OAB).

Two new buildings will anchor the NIF complex (Figure 1-1). The LTAB will house the laser systems, control systems, pulsed power, and target area. The OAB will house clean rooms and optics assembly areas that will be used for final cleaning and inspection of the NIF optical components and subsystems. Additional LLNL facilities and general site infrastructure will support the NIF's ongoing operations. 


\subsection{Pollution Prevention and Waste Minimization}

Pollution prevention (P2) is a system overview focusing on the use of materials, processes, and practices that eliminate or reduce the release of pollutants, contaminants, hazardous substances, and waste into the land, air, or water. P2 analysis is routinely performed on the waste streams from operating facilities; however, such analysis can be significantly more effective when done during the design phase of a facility. P2 includes practices that eliminate or minimize the discharge of all wastes, including hazardous, radioactive, or toxic materials, to the environment. It also includes systems to protect natural resources through conservation and improved efficiency. P2 reduces the amount of energy and water consumed, and the quantity of hazardous materials used.

Assessed alone, P2 represents the first step in a hierarchy of options for managing waste. P2 attempts to eliminate waste before it is generated. Waste minimization (WMin), the minimization of waste streams once they are being generated, represents the next level in a waste management hierarchy. The total environmental protection hierarchy lists (in descending order of preference) source reduction, recycling, treatment, and disposal as the recommended options for waste management. Source reduction is assigned the highest priority because it eliminates or reduces wastes at the source of generation. Recycling is the next-preferred approach because it involves the reuse or regeneration of materials and wastes into usable products. Treatment and disposal are last-resort measures.

\subsection{Purpose of the NIF P2/WMin Plan}

The purpose of this NIF Pollution Prevention and Waste Minimization Plan (P2/WMin Plan) is to incorporate both of these environmental management techniques (that is, pollution prevention and waste minimization) as drivers in the design, construction, operation, and final decommissioning of the NIF. One of the principal goals in the development of this P2/WMin Plan for the NIF project is to exceed compliance with all applicable environmental regulatory requirements, using cost-effective pollution prevention opportunities, by taking a proactive approach to environmental issues. During project design, LLNL researchers from the Pollution Prevention Group (PPG), a group within the LLNL Environmental Protection Department, worked with the NIF staff to review potential waste stream generation. Throughout the operational phase, the NIF staff will continue to work with PPG researchers to review operational waste streams. This collaboration has identified opportunities for P2 and WMin, and it will continue to do so throughout the entire life cycle of the NIF.

This P2/WMin Plan will function as the planning document for anticipating, minimizing, and mitigating NIF waste generation. It is also a DOE milestone document specified in the facility's Mitigation Action Plan (MAP). As such, it is one of the reference documents that will guide NIF operations through all phases. This document will be updated periodically to reflect NIF development, from construction through lifetime operation. 


\subsection{Scope: Roles, Responsibilities, and Goals}

The NIF P2/WMin planning process included a review all of the facility's significant waste-generating processes at each phase of the project. This review included design, construction, and operation, through decontamination and decommissioning (D\&D). Figure 2-1 shows the NIF timeline up to the start of NIF operations. At the time the planning process was initiated, many NIF components were in the preliminary stages (Title I) of design. PPG researchers therefore applied a predictive pollution prevention opportunity assessment (PPOA) methodology to assess anticipated waste streams. In several instances, significant costs were reduced, or eliminated entirely, by optimizing NIF designs for minimal environmental impact. These optimizing designs do not interfere with the performance considerations or budget constraints of the NIF project.

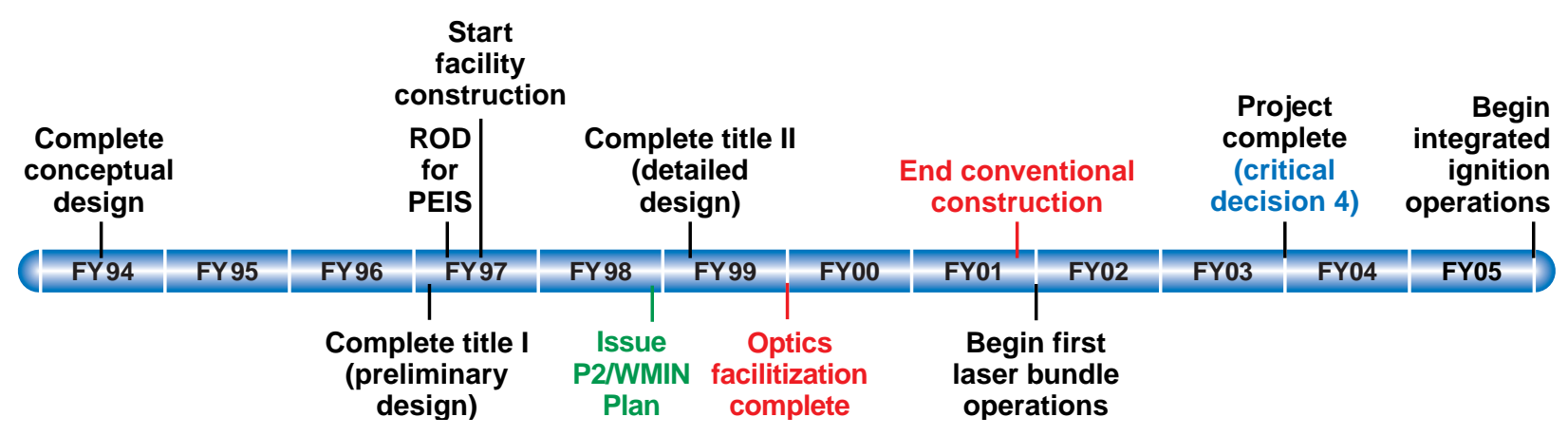

Figure 2-1. NIF timeline from conceptual design to start of NIF operations.

The NIF project team and researchers from PPG assembled this plan as a joint effort. During investigations undertaken for the plan, the NIF program demonstrated a genuine commitment to minimize the environmental impact of the project to the full extent technically feasible and economically possible. The primary waste streams evaluated in predictive PPOAs were identified in the Final Programmatic Environmental Impact Statement for Stockpile Stewardship and Management (PEIS) issued by DOE (DOE/EIS-0236, September 1996). In addition, waste minimization activities benefiting the construction phase are included in this plan.

Energy consumption anticipated for the operational phase of the NIF has been discussed in two specific publications: Laser $\mathcal{E}$ Target Area Building Conventional Facilities Energy Conservation Report (NIF-0004309) and NIF Optics Assembly Building Title II Design Energy Conservation Report (NIF-0010433). The results are briefly summarized in Section 4.3 of this document. For a detailed analysis of the findings, consult the appropriate NIF publication. 
Similarly, the LLNL site-wide Storm Water Pollution Prevention Plan (SWPPP) discusses overall NIF management of storm water discharges during operation (see Section 4.2.1.1 of this document). However, SWPPPs were developed and followed for various and unique activities on the NIF site during the construction phase of the facility.

Section 6.0 of this document summarizes the NIF P2/WMin accomplishments in place as of June 1998. The accomplishments include costs savings, waste reductions, and waste stream eliminations. Further accomplishments are expected as the PPOAs are implemented.

\subsection{Environmental Protection}

LLNL Director Bruce Tarter stated the Laboratory's Environmental Safety and Health policy in 1996, as follows:

"It is the Laboratory's ES\&H policy to perform work in a manner that protects the health and safety of employees and the public, preserves the quality of the environment, and prevents property damage. The environment, safety, and health are to be priority considerations in the planning and execution of all work activities at the Laboratory. Furthermore, it is the policy of LLNL to comply with all applicable ES\&H laws, regulations, and requirements."

The NIF P2/WMin Plan contains policies, objectives, and strategies supporting this commitment.

LLNL continually updates the site-wide Pollution Prevention Plan (UCRL-AR127073). The NIF P2/WMin Plan will operate in conjunction with the site-wide plan to enable timely and economical reduction or elimination of waste streams from NIF activities.

\subsection{P2 Roles and Responsibilities}

The LLNL Director establishes Laboratory policy and delegates to the associate directors (ADs) responsibility for implementing Environmental Safety \& Health (ES\&H) policies as an integral part of their management responsibilities. From the ADs, responsibility for implementing these policies continues down through line management to the individual workers. Pollution prevention is incorporated into the ES\&H policies and infrastructure at LLNL.

Except for direction-setting policies generated by the Director's Office, policies related to ES\&H are developed under the guidance of the ES\&H Working Group. One of the ES\&H program's objectives is to establish an environmental protection program that encourages innovative approaches to P2/WMin. 
An unbroken chain of management responsibility and authority extends from the Director of the Laboratory through each AD's organization. Four levels of responsibility and authority characterize this line management chain: executives, senior managers, managers, and supervisors. Line management responsibilities include taking actions to minimize the generation of waste, and enforcing the policies and procedures governing LLNL waste-handling practices; environment-related operating permits; and P2. Line management ensures that both construction and operations are monitored regularly and ES\&H-related problems are mitigated using the principles of graded approach.

Four ES\&H teams provide services and support to programmatic and overhead organizations to help them ensure a safe and healthy workplace. These ES\&H teams are fundamental to a successful implementation of the ES\&H program, and they are regarded as the backbone of the organizational framework. ES\&H Team 2 supports the Lasers Directorate and NIF construction, activation, and operations.

In the current stages of the NIF (design and construction), all environmental matters including oversight of the development of this plan are within the purview of the NIF E\&S Working Group. This working group ensures that facility activities interface with all appropriate environmental personnel external to the NIF project organization, and that all appropriate environmental permitting and planning occur in a timely fashion. The DOE Oakland Operations Office (DOE/OAK) has a representative on the NIF E\&S Working Group.

\subsection{Regulatory Compliance}

\subsubsection{Full Compliance with Regulatory Agency Requirements}

The NIF P2/WMin Plan specifies activities and methods that are in full compliance with all applicable regulations and agency reporting requirements. Whereas many federal requirements exist to implement $\mathrm{P} 2$ within the $\mathrm{DOE}$, it is important to recognize that the primary reason for $\mathrm{P} 2$ is that it is both good environment practice and good business practice.

\subsubsection{PEIS as the Baseline}

Waste will be categorized as nonhazardous, hazardous, radioactive, or mixed, as identified in the PEIS baseline. The PEIS was based on 1994 estimates using the state of the NIF design at that time. Waste streams will be reevaluated prior to startup. DOE Orders 5400.1, General Environmental Protection Program, and 5820.2A mandate that the management of hazardous, radioactive, and mixed wastes shall be accomplished in a manner that minimizes the generation of such wastes. The NIF P2/WMin accomplishments in this area to date are addressed in Section 6.0 of this document. 


\subsubsection{Federal Mandates}

The United States Congress, through the enactment of the Pollution Prevention Act of 1990, established P2 as the preferred approach for managing environmental releases. The Act establishes source reduction as the national strategy of first choice to reduce the generation of pollution.

To emphasize the importance of P2, on August 3, 1993, President Clinton issued Executive Order 12856, Federal Compliance With Right-to-Know Laws and Pollution Prevention Requirements. The Executive Order encourages P2 leadership within the federal government. It directs all federal agencies to develop goals to reduce by $50 \%$ their total releases to the environment and their off-site transfers for treatment and disposal of toxic chemicals regulated under the Emergency Planning and Community Right-to-Know Act (EPCRA), Section 313. The deadline for implementing the 50\% reduction is December 31, 1999. In addition, each federal agency must review its specifications and standards, and identify opportunities to eliminate or reduce the use of toxic chemicals. Furthermore, each agency and each facility within that agency required to comply with EPCRA Section 313 must have a plan with goals to eliminate or reduce the unnecessary acquisition of products containing toxic chemicals.

As required by Executive Order 12856, the Secretary of Energy, on December 28, 1994, issued DOE's Pollution Prevention Strategy (2), to be implemented by all departmental elements. This document establishes P2 as the DOE's primary strategy to reduce the generation of all waste streams and thereby minimize the impact of departmental operations on the environment, reduce operational costs, and improve energy efficiency and safety.

Executive Order 12873, Federal Acquisition, Recycling, and Waste Prevention, October 1993, directs the appointment of federal agency environmental executives to develop and implement acquisition programs aimed at encouraging new technologies and to build markets for environmentally preferable and recycled products. Federal agencies also must set goals for waste reduction, recycling, and the acquisition of recycled products, and then they must report on their progress in meeting these goals.

The Resource Conservation and Recovery Act (RCRA) requires hazardous waste generators to establish a program to reduce the volume or hazardous properties of waste to the degree determined by the generator to be "economically practicable." Hazardous waste generators must certify in their waste manifests that this requirement has been fulfilled. Generators must also identify in their biennial reports to the Environmental Protection Agency (EPA), and in many cases to their respective state and local environmental regulatory agencies, their efforts undertaken during the year to reduce the volume and hazardous properties of generated wastes.

\subsubsection{State Mandates}

In California, the Hazardous Waste Source Reduction and Management Review Act of 1989, or Senate Bill 14 (SB 14), requires documentation of existing and proposed 
WMin programs. Senate Bill 14 requires facilities that generate large amounts of hazardous waste to document their existing and proposed waste reduction measures. Under SB 14, every four years, facilities must report on the progress of waste minimization activities, changes in waste management activities, and evaluation of waste reduction alternatives. Facilities must also develop plans for implementing future waste reduction measures.

The California Department of Toxic Substances Control (DTSC) also regulates management of, as well as reduction of, hazardous wastes in California. Transportation waste manifests used in California include a waste minimization certificate signed by responsible generating facility personnel. California Code of Regulations (CCR) Title 22, Section 66262.20 and its Appendix, include the specific requirements for hazardous waste manifests. Similarly, the DTSC regulates the preparation and submittal of Hazardous Waste Biennial Reports, which must document the effectiveness of the hazardous waste generator's waste minimization programs.

California Assembly Bill 939 (AB 939) was enacted in 1989. It requires cities and counties to reduce their 1990 solid-waste-stream levels by $25 \%$ by 1995 , and $50 \%$ by 2000. AB 939 details the reporting requirements for cities and counties as well as preparation of plans for achieving the goals discussed above. AB 939 also includes a structure for fining cities and counties that do not make an effort to comply.

\subsubsection{Regional Agencies}

The EPA establishes National Ambient Air Quality Standards (NAAQS). California delegates responsibility for attaining the NAAQS to Air Pollution Control Districts (APCDs) and Air Quality Management Districts (AQMDs). The LLNL main site is regulated by the Bay Area Air Quality Management District (BAAQMD). The BAAQMD was created to develop and administer air pollutant control programs for the San Francisco Bay Area. The BAAQMD specifically focuses on controlling air pollutants from stationary sources and implementing standards for the San Francisco Bay Area and surrounding vicinity. The general objective of these activities is to attain compliance with the Federal Clean Air Act (and amendments), the California Clean Air Act (CCAA), and other state regulations that control emissions of many common (or criteria) air pollutants and toxic air contaminants.

In California, sources of air pollution are largely regulated through a permit system and the application of prohibitory rules. The permit system is designed to permit each piece of equipment, group of equipment, or process that may emit air pollutants on an individual basis. Permits may be required for new equipment or operations that may cause air pollution and for modifications to equipment, throughputs, processes, or materials. The State of California has also established its own California Ambient Air Quality Standards (CAAQS) along with the CCAA, both of which are more restrictive than federal requirements. 


\subsubsection{County Agencies}

The Alameda County Flood Control and Water Conservation District, Zone 7, is responsible for administering and controlling local water resources. The district develops and manages flood control structures and works to ensure the protection of area resources against flood damage.

\subsubsection{City and Municipal Agencies}

The City of Livermore Water Reclamation Plant (LWRP) is responsible for treating and managing the waste water generated and collected by the municipal sewer system. This facility also ensures the implementation of waste water pretreatment standards from industrial sources and manages the city's effluent discharge in accordance with applicable water quality standards set forth by the Regional Water Quality Control Board.

Sanitary sewer discharges at LLNL are regulated under a site-wide Wastewater Discharge/Chemical Storage permit from the local control authority. LLNL must annually submit a comprehensive permit application to the LWRP for discharge permit renewal. Not all discharges are released directly to the sewer. Some process waste streams are first contained and treated prior to discharge.

\section{$2.4 \quad$ NIF P2/WMin Goals}

In keeping with the priorities of the LLNL site-wide P2 program, the NIF has an ongoing commitment to four immediate priorities for reducing waste generation and environmental impacts:

- Compliance with all applicable federal, state, regional, and local environmental regulations.

- Integration of P2/WMin strategies into the NIF design.

- Accurate forecasting of waste streams.

- Implementation of technically and economically feasible P2/WMin measures.

\subsubsection{Goal Implementation Strategies}

\subsubsection{Design for Environment}

The NIF project management supported an evaluation of the Design for Environment (DfE) activities that produced the initial development of this P2/WMin Plan; development of construction-phase recycling plans; analysis of some of the specialized materials of construction to minimize future D\&D costs; and development of cost assessments for more benign cleaning procedures that meet the NIF's stringent specifications. 
The principles of predictive assessment and the proactive approaches embodied in this initial DfE analysis will extend throughout the facility's lifetime and can be included in the planning stages of any major changes in NIF activities, architectures, or tasks. Such a process can be embodied in a "front end" review process that applies to new projects or experiments that could have a significant impact on the environment. In this review process, the initial hazardous materials projected to be used can be identified, and concentrations of both the starting materials and the wastes produced are estimated. The possibility for chemical substitution, process changes, and recycling is then addressed. If an opportunity for P2 is identified, the PPG can assist in evaluating options.

\subsubsection{Waste Stream Analysis and Prioritization}

The NIF waste streams will be periodically evaluated and ranked in order of greatest concern. Current LLNL methodology assigns to each waste stream three weighting factors in addition to a factor based on quantity of waste generated annually. The three weighting factors use the following criteria: cost, waste type (including compliance and liability considerations), and operational aspects such as routine versus nonroutine. The weighting scheme is discussed in Reassessment of LLNL Waste Generation for Calendar Year 1995 (UCRL-AR-125047).

Once the factors have been prioritized, cost/benefit methodologies will be applied to the evaluation of $\mathrm{P} 2$ opportunities for the prioritized waste streams. These assessments will guide the allocation of funding for P2 measures at the NIF.

\subsubsection{Awareness Training for Building Coordinators}

As at all LLNL facilities, building coordinators will be trained to appraise the effectiveness of P2/WMin practices. During the NIF operational phase, building coordinators will actively seek additional ways to reduce, reuse, and recycle whenever possible.

\subsubsection{Awareness Guidelines for NIF Personnel}

P2/WMin guidelines will be provided for NIF personnel, beginning with a description of regulatory drivers and noting the shift from pollution control to pollution prevention. DOE Orders requiring the minimization of waste during facility design will be specifically noted. Definitions of terms and the P2/WMin hierarchy will be presented. 


\subsection{NIF P2/WMin Planning}

The NIF P2/WMin planning occurs within the overall context of the Pollution Prevention Program at LLNL. That program has six established priorities.

1. Establishing senior management's commitment to P2/WMin implementation.

2. Quantitative, site-specific waste reduction and recycling goals.

3. Performance measures.

4. Implementing cost-saving P2/WMin projects.

5. Designing P2/WMin into new products, processes, and facilities.

6. Ensuring that site programs comply with federal, state, and DOE requirements.

Because the NIF is currently in the design and construction phase, those elements are receiving the most attention. Therefore, unique NIF issues are the subject of current focus. Most of the unique issues have evolved from the DfE planning discussed below. Predictive PPOAs (those based on predictions of waste stream components identified in a design) foster an understanding of the front end (the process producing the waste stream) and assist in identifying a range of potential options engineers can study during project design.

\subsection{Design for Environment (DfE) Planning}

One of the goals of the NIF project is to meet, and when possible exceed, compliance with all applicable environmental regulatory requirements by taking a proactive approach to environmental issues. In the design phases of a project, a DfE study can generate a prioritized set of options that project management can consider for further study. From the set of prioritized options, a strategy can be developed by which the project can approach an environmentally proactive stance.

DfE planning is a nascent discipline, with several emerging interpretations and methodologies. In general, all means of minimizing environmental life-cycle impacts can be considered elements of DfE planning. Specific environmental impacts of the NIF can be minimized in four ways:

- Implementing pollution prevention, energy efficiency, and other resource conservation measures in the design phase.

- Developing plans for using less toxic and nontoxic materials, and minimizing hazardous and radioactive waste generation.

- Creating facilities (buildings) or experiments that can be disassembled, refurbished for reuse, or recycled.

- Evaluating the environmental costs associated with a process or facility component and system under consideration. 
An initial DfE review was conducted to assess the current state of the entire NIF project ("DfE for the NIF," DOE Pollution Prevention Conference XIV, Seattle, WA, June 1-4,1998). Using a combination of traditional project management techniques and a DfE perspective, the review focused on those design elements most likely to be influenced by a DfE perspective. This process helped focus on those environmental benefits with the highest priority for the NIF project.

\subsection{Construction-Phase Planning}

All potential construction-phase environmental impacts have been evaluated, and mitigation measures have been developed, as necessary. The mitigation measures developed and in place for the NIF construction are extensive.

- Surveillance and sampling plans have been developed.

- Land use plans include land restoration following construction, where appropriate (e.g., the construction laydown area will be restored with a landscape design).

- Air-quality control plans include appropriate dust-control measures, such as water spraying or chemical dust suppression. Air pollutants from fuel combustion and solvent use are appropriately permitted and controlled.

- Construction soil recycling plans have been developed to reuse soil excavated during construction. Soil is reused on-site whenever possible. These procedures are currently in place, and more than 281,000 metric tons of construction soil have already been reused. The NIF contractors are required to forecast excess soil generation and provide receipts for the actual amounts taken to landfills.

- Construction has proceeded under several Storm Water Pollution Prevention Plans (SWPPPs) issued pursuant to California's general National Pollutant Discharge Elimination System (NPDES) permit requirements for storm water discharges associated with construction activities. In addition, a successful spillprevention program is being conducted per the LLNL Spill Prevention Control and Countermeasures (SPCC) plan. Secondary containment, offsite fueling operations, and dry-type transformers are all elements of the NIF construction mitigation measures.

\subsection{Activation-Phase and Operational-Phase Planning}

During these phases, the NIF WMin activities will seek to avoid or reduce the generation of operational waste streams, to reduce the hazardous properties of waste streams that cannot be eliminated, and to maximize recycling efforts.

Once the NIF is generating wastes as an operating facility, a successful P2/WMin program is critical to continue reducing waste treatment and disposal costs, to anticipating and developing continuous improvements in environmental protection and worker safety, to anticipating more restrictive future regulatory requirements, and to demonstrating the NIF commitment to the public and to the environment. 
A vital P2/WMin plan for the NIF in its operational phases must include consideration of awareness and training for startup personnel who make the transition to operations personnel. Sections 2.4, 4.5, and 4.6 describe an initial P2 planning outline for the facility as it moves from design through construction and activation, anticipating operational issues as they become better understood. As this plan matures to become a functional document for an operational NIF, it will incorporate the following important functions:

- Outline programmatic and management responsibilities for P2/WMin.

- Develop and implement P2/WMin employee awareness and training.

- Develop and implement the means for monitoring waste generation within NIF.

- Use monitoring information together with operator knowledge to forecast changes in waste generation as the NIF operations evolve throughout its lifetime.

- Assign personnel to implement P2/WMin measures as such options are available and economically feasible.

- Award and otherwise recognize NIF employee efforts to effect P2/WMin in NIF operations.

\subsubsection{PPOAs for Operational Waste Streams}

LLNL and NIF personnel recognize their public responsibility to reduce future environmental consequences arising from the waste generated by facility operations. Once the NIF is operational, opportunity assessments will help identify cost-effective techniques for reducing or eliminating potential wastes.

To assess operational waste streams for reduction or elimination, the NIF will follow the general procedures for P2 opportunity assessments as outlined in the DOE performance-based course "Pollution Prevention Opportunity Assessment." A synopsis of this procedure is contained in Appendix A.

\subsubsection{Tracking Methods}

All waste steams will be managed in accordance with federal, state, and local regulations, DOE orders, and LLNL policies. Personnel from various groups within the EPD will assist NIF personnel in the conduct of environmentally responsible management of the waste streams. The NIF will implement a means of monitoring its waste generation, and the HWMD Total Waste Management System will establish database information about NIF wastes requisitioned for storage, treatment, or disposal. ChemTrack monitors the movement and consumption of chemical products at LLNL. The Chemical Exchange Warehouse (CHEW) can help programs identify reuse options for excess products. 


\subsection{Decontamination and Decommissioning Phase Planning}

Decontamination and decommissioning (D\&D) actions are taken at the end of a facility's lifetime to remove radioactive and hazardous contamination and to reduce health and safety impacts during removal or in preparation for reuse of the facility's components. The NIF Decontamination and Decommissioning Plan (NIF-0001670, WBS 1.1.3) addresses the decommissioning of the laser and target areas and associated NIF systems at the end of the program's lifetime. The D\&D Plan is periodically updated, and reviews of the Plan several years prior to initiation of such activities may significantly improve further P2 planning for D\&D. Beamlet and Nova decommissioning experience can be used to develop P2/WMin planning for the NIF D\&D. It is anticipated that most of the nonactivated metals, concrete, and structural steel in the NIF will be reused (refer to Section 4.5.1, Figure 4.1).

Two radiological issues dominate decommissioning at the NIF end of life. They are the extent of tritium contamination and the contact dose rate due to long-lived activation products induced in components and large structures. Several facility features will minimize these issues at the end of life:

- Confinement barriers and ventilation control will prevent or minimize the spread of tritium throughout the facility.

- Special surface coatings, such as epoxy paint on walls, ceilings, and floors, will provide an effective barrier against tritium sorption.

- Use of low-activation materials, such as the aluminum target chamber and supporting structures, and low-activation concrete, will minimize activation.

Selection of concrete for the NIF floors and walls has been based on minimizing activation of materials in the concrete over the lifetime of the facility. Smaller components, such as the target positioner and diagnostics, will have been designed to be constructed from minimally activating materials as well.

Operational procedures will also minimize the extent of contamination. For example, components removed from the target chamber will be properly packaged, and PPE will be used in contaminated areas. Experimenters will be instructed to minimize the total target mass and to carefully select target materials. Periodic cleaning of equipment exposed to tritium and activated debris will maintain low contamination levels throughout the facility lifetime. Such practices will significantly reduce, if not virtually eliminate, the need for major end-of-life decontamination. Cleaning and decontamination technologies have been developed to meet NIF decontamination needs. Cleaning methods are likely to include:

- Vacuum bake-out, water rinse, and dilute acid wash for debris shields.

- High-pressure water spray, ultrasonic bath in water and surfactants, and oven drying for first-wall panels.

- Aqueous wipe-down for small diagnostics.

- Carbon dioxide snow cleaning for large diagnostics with complex geometries. 
NIF-0010575

UCRL-AR-131194

NIF decommissioning operations will make every effort to reuse and recycle all portions of the target area. From current understanding and analysis, decommissioning the NIF will not present major difficulties. It should be possible to return the facility to unrestricted use. 


\subsection{Implementation of Waste Stream Monitoring, WMin Assessments, and P2 Opportunity Methods}

\subsection{Waste Stream Monitoring and Management}

Waste stream monitoring and management fall within the purview of the Environmental Protection Department (EPD) at LLNL. The EPD prepares and maintains environmental plans and guidelines, and it provides environmental guidance and support to LLNL personnel. Within the EPD, the Operations and Regulatory Affairs Division (ORAD) prepares environmental permit applications; tracks chemical inventories; conducts compliance and surveillance monitoring; and provides environmental impact modeling and analysis, risk assessment, and reporting. The Hazardous Waste Management Division (HWMD) manages all hazardous, radioactive, and mixed wastes generated at LLNL facilities. The HWMD processes, stores, packages, solidifies, treats, and prepares waste for shipment, disposal, recycling, or discharge to the sanitary sewer. All LLNL environmental management activities are summarized each year in the Site Annual Environmental Report (SAER) (UCRL-50027-96, 1996 edition).

\subsection{Waste Stream Minimization and Reuse}

The P2/WMin Plan, and all waste stream minimization and reuse for the NIF, will have the same scope as that of the P2 program at LLNL. This program is an organized, comprehensive, and continuing effort to systematically reduce solid, hazardous, radioactive, and mixed waste generation. The P2 program is designed to eliminate or minimize pollutant releases to all environmental media from any aspect of LLNL operations. These efforts offer increased protection of public health and the environment, and they will yield additional benefits by reducing or eliminating:

- Waste management and compliance costs.

- Resource usage.

- Inventories and releases of hazardous chemicals and radioactive materials.

- Civil and criminal liabilities under environmental laws.

\subsubsection{Nonhazardous Waste Stream Minimization and Reuse}

Nonhazardous solid waste stream diversion has been highly successful at LLNL and is incorporated into the NIF planning. LLNL now diverts reusable/recyclable goods, rather than sending them to landfills. Reused materials include asphalt, batteries, cardboard, compost, and metals. Paper, office supplies, and tires are also successfully recycled. Recycled NIF soil accounted for an additional $>281,000$ metric tons of reused material in 1997, and it is anticipated to account for an additional 27,300 metric tons of 
reused material in 1998. In addition, 812 metric tons of concrete and asphalt from the NIF project were reused in 1997. Materials such as scrap metal and wood will be recycled upon the start of associated construction activities commencing in 1998. Source reduction also plays an important role in checking waste generation.

\subsubsection{Storm Water Runoff Management}

As required by the Federal Clean Water Act, storm water management during NIF construction proceeds under the guidance of a state-wide, general NPDES general permit for the discharge of storm water. During its operational phase, the NIF will be managed for storm water as part of the larger LLNL plant under the existing NPDES permit for storm water and low-threat nonstorm water discharges. During operation, the NIF will implement the LLNL Storm Water Pollution Prevention Plan (SWPPP).

Storm water runoff during NIF construction activities is routed to the LLNL storm drainage system. Retention structures contained water that fell onto the NIF site and that was pumped out of the excavations. This water was impounded to allow sediment to settle out of the water prior to release to the storm drainage system. The collected runoff was also tested for $\mathrm{pH}$ following application of lime to stabilize construction roads during the 1997-98 rainy season. The $\mathrm{pH}$ of the water had to fall within a range of 6.5 to 8.5 to qualify for release to the storm drainage system.

During the construction phase of the NIF, three SWPPPs apply to the conventional facilities: National Ignition Facility Construction Storm Water Pollution Prevention Plan; National Ignition Facility Construction Storm Water Pollution Prevention Plan, "Addendum, Optics Assembly Building;" and National Ignition Facility Construction Storm Water Pollution Prevention Plan, "Addendum, Greenville Road Crossing."

Three additional SWPPPs pertain to special equipment: National Ignition Facility Construction Storm Water Pollution Prevention Plan, "Addendum, Target Chamber Assembly Building Staging Area"; National Ignition Facility Construction Storm Water Pollution Prevention Plan, "Addendum, Building 298 Laydown Area"; and National Ignition Facility Construction Storm Water Pollution Prevention Plan, "Addendum, T4900 Staging Area." These SWPPPs must be updated, or newly created, whenever there is a significant change in construction activity that may affect the quality of storm water discharges. The SWPPPs may also require updating when California renews the NPDES permit, which is required every 5 years.

\subsubsection{Air Emissions Minimization}

LLNL performs all activities necessary to comply with federal, state, and local cleanair requirements. Air quality is protected during all phases of the NIF life cycle in accordance with the regional air management district guidelines and National Emission Standards for Hazardous Air Pollutants (NESHAP) requirements. Monitoring is performed by the EPD for radionuclides pursuant to NESHAP and for beryllium pursuant to air district guidelines. Whenever required, air permits are issued through 
the BAAQMD. LLNL conducts self-monitoring and inspections associated with the requirements of these permits.

During the NIF construction phase, air quality impacts are being actively minimized. The greatest impact during construction has been fugitive dust (PM10) emissions. To date, NIF subcontractors have used two dedicated water trucks during site preparation to minimize impacts on air quality. Geotextile materials were used to cover some exposed areas, and stockpiles were managed to reduce exposure to wind. Excavation of the main facility is complete, and the utility trenches are now being excavated. Depending on meteorological conditions, conventional water spraying for dust control, or additional dust control measures that use continuous water spraying (e.g., dedicated water truck), or chemical dust suppressants will continue to be used to minimize impacts on ambient air quality.

The incorporation of alternatives to the use of solvents in the design phases of the NIF (see Section 5.0 of this document), will result in significant decreases in volatile organic compound emissions for the NIF, when compared with historical operations for other facilities.

\subsection{Energy Efficiency Assessments}

The NIF Scope of Work for Architect-Engineering Services required that preliminary energy-conservation reports be completed (e.g., National Ignition Facility Laser \& Target Area Building Conventional Facilities Energy Conservation Report, NIF-0004309). Various energy-conservation alternatives were evaluated on a life-cycle cost basis for implementation in the final design effort. The NIF buildings are designed to comply with 10 CFR 435, "Energy Conservation Voluntary Performance Standards."

\subsubsection{NIF Laser and Target Area Building Conventional Facilities Energy Conservation Report}

The $45,744 \mathrm{~m}^{2}\left(492,403 \mathrm{ft}^{2}\right)$ LTAB building is the main experimental building of the NIF. It will consist of two laser bays, four capacitor bays, two optical switchyards, a target bay, an attached diagnostics building, a decontamination area, and operationalsupport areas. The National Ignition Facility Laser E Target Area Building Conventional Facilities Energy Conservation Report (NIF-0004309) compared a "base case" building to nine design alternatives for the LTAB. The Carrier E20-II Engineering Economic Analysis computer program, version V2.10, was used to evaluate the costs and benefits of potential energy conservation projects and to determine which has the lowest lifecycle costs for the LTAB.

\subsubsection{Cases Analyzed}

The base case building used R-13 insulation for walls and R-19 insulation for the roof. The base case water-cooled centrifugal chillers had an efficiency of $0.587 \mathrm{~kW} /$ ton and fiberglass cooling towers with PVC fill. The alternative cases considered variations 
in the amount of building insulation, the efficiency of water chillers, the water-supply and water-return temperatures of cooling towers, or a combination of these parameters, as summarized in Table 4-1.

Table 4-1. Description of LTAB energy-conservation alternatives assessed.

\begin{tabular}{|l|l|}
\hline \multicolumn{1}{|c|}{ Case analyzed } & \multicolumn{1}{c|}{ Parameter values } \\
\hline Base case insulation & $\mathrm{R}=13$ (walls), $\mathrm{R}=19$ (roof) \\
\hline Alternative 1 insulation & $\mathrm{R}=19$ (walls), $\mathrm{R}=30$ (roof) \\
\hline Base case chiller & efficiency $=0.587 \mathrm{~kW} /$ ton \\
\hline Alternative 1 chiller & efficiency $=0.563 \mathrm{~kW} /$ ton \\
\hline Alternative 2 chiller & efficiency $=0.513 \mathrm{~kW} /$ ton \\
\hline Base case cooling towers & condenser water supply $=28.3^{\circ} \mathrm{C}$; water return $=35^{\circ} \mathrm{C}$ \\
\hline Alternative 1 cooling towers & condenser water supply $=29.4^{\circ} \mathrm{C}$; water return $=35^{\circ} \mathrm{C}$ \\
\hline Alternative 2 cooling towers & condenser water supply $=29.4^{\circ} \mathrm{C}$; water return $=36^{\circ} \mathrm{C}$ \\
\hline Combined alternatives 1 & base case cooling tower + alternative- 1 chiller \\
\hline Combined alternatives 2 & alternative-2 cooling tower + base case chiller \\
\hline
\end{tabular}

\subsubsection{Analysis Results}

The proposed heating, ventilating, and air-conditioning (HVAC) systems of the LTAB are dictated by the unique requirements and stringent design criteria of the NIF. From the 30-year, life-cycle cost analysis of various cases analyzed, the following energy-conservation modifications were recommended for implementation in the Title II final design:

- Base case insulation.

- Alternative 1 chiller.

- Base case cooling towers combined with alternative 1 chiller.

The recommended design provides an energy-efficient building exceeding the maximum requirements. For more details on the results of this analysis, refer to the National Ignition Facility Laser \& Target Area Building Conventional Facilities Energy Conservation Report (NIF-0004309, WBS 1.2.1).

\subsubsection{NIF Optics Assembly Building Title II-Design Energy Conservation Report}

The $\mathrm{OAB}$ is a two-story building with a basement and adjoining areas that will support the laser experimental activities in the LTAB by providing optics assembly and refurbishment. Total conditioned space in the facility will be approximately $2,020 \mathrm{~m}^{2}$ $\left(21,750 \mathrm{ft}^{2}\right)$. The main operation in support of the LTAB will be to handle all components of the laser system as they are prepared, cleaned, assembled, and aligned in the OAB. The components must be kept very clean, requiring clean rooms of Class 100,000 to 
Class 100 throughout the facility. A large volume of air will be supplied at ceiling level, creating a laminar air flow to "rinse" suspended particles from the air.

The nature of the optics assembly and refurbishment process makes it highly energy intensive. Because of the precise alignments within the system, temperatures in the clean rooms must remain highly stable at $20^{\circ} \mathrm{C} \pm 0.3^{\circ} \mathrm{C}$. The relative humidity will be maintained at $45 \% \pm 15 \%$, and the floors will be nearly vibration-free. The facility will operate 24 hours per day, year-round.

\subsubsection{Cases Analyzed}

Three different HVAC cases were analyzed for their overall efficiency.

- Energy Conservation Measure 1. This conventional approach employs a constant-volume fan that mixes outside air with the return air. This mixture then enters the cooling coil, dehumidification coil, and reheating coil. It is supplied through ceiling-level HEPA filters into the conditioned space. Treatment of the clean room supply air can be very costly under this method.

- Energy Conservation Measure 2. To avoid the inefficiencies of cooling and reheating a large volume of supply air, this approach uses a primary air system in which the outside air is conditioned and distributed. A secondary system moves a large amount of supply air via recirculating fans, without requiring any cooling and reheating treatment. The space cooling load and make-up air are provided by the primary system. The large reserve of heat energy from the fans reduces the need for clean room heating, even during winter months.

- Energy Conservation Measure 3. The Measure 3 approach uses a primarysecondary system similar to Measure 2, except that the space-sensible load is handled by cooling coils in the recirculating fans, rather than the make-up air fans. In this case, the make-up air is minimized to the volume required to compensate for exhaust, fresh air, and pressurization needs. Each recirculating fan, in this case, will contain a set of cooling coils to handle the room-sensible load. The make-up air also serves to control the clean room humidity level.

\subsubsection{Analysis Results}

The three HVAC cases were analyzed under comparable operating conditions. The proposed design with the lowest energy consumption and least costs was Energy Conservation Measure 3, thus Measure 3 was implemented. In addition to this finding, further energy conservation considerations are planned for the HVAC design. The building envelope will incorporate R-28 roof insulation and R-19 wall insulation, and electronic ballasts will be installed in lighting fixtures throughout the facility. For more details on this energy conservation analysis, see the National Ignition Facility Optics Assembly Building Title II-Design Energy Conservation Report (NIF-0010433). 


\subsection{Environmental Costs}

Environmental costs have two major dimensions for the NIF: costs that directly impact the project's bottom line, and costs to individuals, society, and the environment for which there is no accounting. Environmental performance is one of the many important measures of operational success. Environmental costs and performance deserve attention for many reasons:

- Environmental costs can be significantly reduced or eliminated as a result of informed management decisions. (Operational and maintenance changes and process redesign as technology matures to meet project needs are just two examples.)

- Environmental costs (and their areas of potential savings) may be obscured in overhead accounts or otherwise overlooked. Some potentially hidden environmental costs include personnel for regulatory reporting, training, monitoring, and data management; community perception costs; and unanticipated future costs resulting from current waste management decisions.

- Better management of environmental costs can result in improved environmental performance and significant benefits to human health.

Data to calculate the NIF life-cycle costs are not yet available. However, one way for the NIF to calculate and manage its environmental costs is to conduct PPOAs before implementing new processes and facilities. The purpose of PPOAs is to characterize waste streams and to identify those P2 options that can be cost-effectively implemented. Included in the PPOA methodology to be used for the NIF during its operational phases is a return on investment (ROI) calculation and cost assessment of the options. The NIF will use ROI calculations and estimates of environmental cost-effectiveness to prioritize P2 projects for resource allocation and implementation.

\subsection{Reuse and Recycling Methods}

\subsubsection{Construction Phase Programs}

Existing buildings, facilities, and resources have been incorporated into the NIF. As an example, part of a Laser Program building is being refurbished to serve the NIF mission, saving the materials, time, and expense of constructing new operating space. Soil excavated during construction of the NIF is conserved for site balancing elsewhere at LLNL and for landscaping and final grading at the NIF to ensure proper storm water management. During construction of the NIF, scrap wood, scrap metal, excess soil, and excess asphalt are being diverted for beneficial reuse or recycling. Figure 4.1 is a summary of current and projected amounts of recycled construction materials associated with the NIF. 


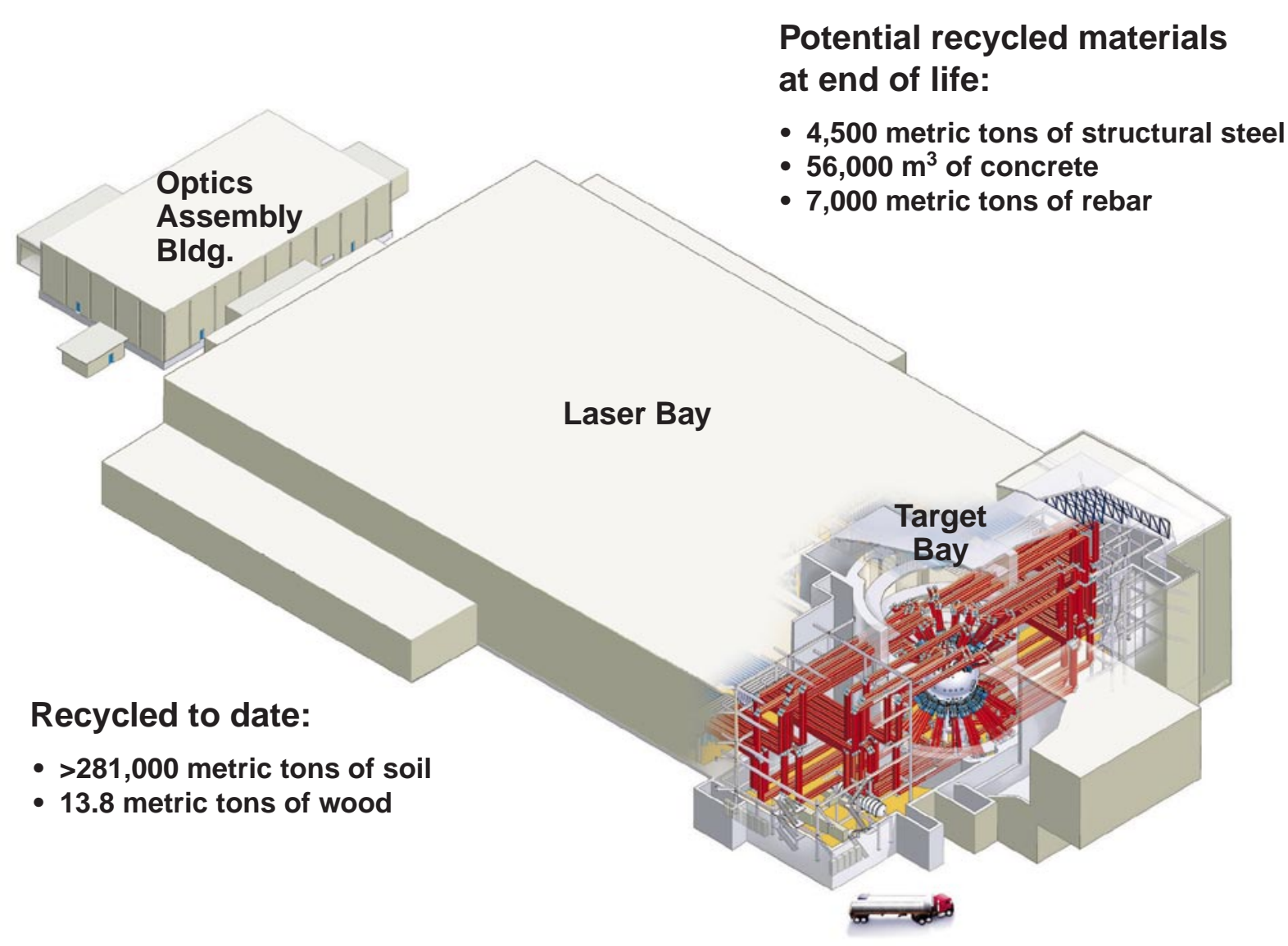

Figure 4.1. Major NIF construction materials for recycling.

\subsubsection{Scrap Wood}

Scrap wood, primarily from concrete-form work, is staged at a designated area within the construction site and picked up when the bin is full. Unrecoverable wood is burned offsite to generate electrical power.

\subsubsection{Scrap Metal}

Scrap steel and other metals are salvaged by vendors who reuse or recycle them as their conditions allow. Contract vendors place bins at specified locations on the NIF construction site. Upon notification, the LLNL vendor will collect the bins and place empty ones as needed.

\subsubsection{Excess Soil}

Construction management reuses as much soil as possible. Excess soils are tested for hazardous constituents and, if acceptable, are then released for use in other LLNL projects. Soil exceeding such needs is delivered to local landfills for daily cover. Site winterizing requires the construction of storm water berms and other retention 
structures. Soil from the NIF excavations is used to temporarily impound rain water until the water can be visually examined, tested if necessary, then released.

\subsubsection{Excess Asphalt and Concrete, Aerosol Can Reuse}

In preparation for the NIF, parking lots and walkways in the construction area were removed. The resulting concrete and asphalt were sent offsite for reuse as roadbed material. Aerosol cans used during NIF construction are recycled through an outside contractor and are either reused or converted into materials for other purposes.

\subsubsection{Activation and Operational Phases}

During its activation and operational phases, the NIF will follow the reuse and recycling policies in effect site-wide at LLNL. Some of the reusable or recyclable materials include paper and packaging, newspaper, magazines, cardboard, and metal. Operational materials specific to the program (e.g., cleaning solvents) will also be reused or recycled when possible. For example, spent cleaning solvents (e.g., toluene and ethanol) may be reusable in other LLNL operations, such as craft shops and plating shops.

\subsubsection{Paper, Cardboard, and Optics Packaging Recycling}

As the NIF facility begins operation, the newly assigned Facility Manager or his designee will order internal paper recycling receptacles from a vendor. Weyerhaeuser Paper Co. (the current vendor for LLNL paper recycling) will place exterior, $1.5-\mathrm{m}^{2}$ paper recycling bins at strategic locations designated by LLNL.

Cardboard is currently picked up by LLNL personnel, then transported and sold to a vendor.

Much of the optics packaging will also be reused. Shipping containers in which NIF optics arrive from vendors will be sent back for reuse, as will the PET-G containers (see Section 5.10.1). The clean nylon and polyethylene optics-bagging material will be recycled as well.

\subsubsection{Metal Recycling}

Nonhazardous scrap metals generated through routine building operations will be handled through the standard LLNL Donation, Utilization, and Sales (DUS) program. The DUS will supply salvage material bins that will be transported to an on-site storage yard for processing when filled by LLNL personnel.

\subsubsection{Decontamination and Decommissioning Phase}

At the time of D\&D, the NIF will be evaluated for site reuse. Any resulting D\&D activities will be conducted only after a PPOA evaluates potential reuse and recycling opportunities. 
The P2/WMin procedures associated with D\&D are discussed in the NIF Decontamination and Decommissioning Plan (NIF-0001670, WBS 1.1.3). To the highest degree economically feasible, NIF components will be reused (e.g., optics and controls), excessed, or salvaged at the end of the project's life.

\subsubsection{Affirmative Procurement}

The EPA has listed procurement guidelines for environmentally preferable products. Wherever possible, LLNL already purchases items that use recovered or recycled material, such as copy paper, publication paper, toner cartridges, and moving boxes. The NIF project has a current and continuing commitment to apply affirmative procurement principles when appropriate. To date, the single, most significant procurement of recycled material recommended on the EPA list by the NIF has been fly-ash-containing concrete. Although technical specifications do not allow the use of fly-ash-containing concrete for all of the NIF's concrete needs, it has been used where possible in areas where neutron shielding is not necessary.

\subsection{NIF P2/WMin Review and Methods}

During operation, nonhazardous NIF wastes will not differ substantially from those already occurring at LLNL. Hazardous liquid wastes will result from normal cleaning and maintenance operations, and they will consist primarily of solvents, acids, paints, and oils. Hazardous solid wastes will consist primarily of hardware that has failed or is beyond its useful life, with no possibility for recycling or reuse. Low-level radioactive wastes will originate in the target chamber and from any cleaning activities associated with components of or insertions into the target chamber. Nonhazardous water-based cleaners used to decontaminate surfaces, molecular-sieve media, wipes, and personal protective equipment (PPE) from decontamination operations and high-efficiency particulate air (HEPA) filters will comprise the low-level waste (LLW) stream. Mixed waste streams will also be generated primarily by decontamination operations using solvents that become metal contaminated and by debris-shield cleaning operations.

Experiments using depleted uranium are currently being considered. (The term "depleted uranium" refers to uranium consisting of $99.7 \%$ uranium-238, the byproduct of the enrichment process that removes U-234 and U-235 from natural uranium.) In this nonyield experiment, approximately 0.1 gram of depleted uranium would be used. During a shot, the depleted uranium would be vaporized. As with other relatively heavy elements used in the chamber, depleted uranium would be deposited on the target chamber walls and on target chamber diagnostic hardware. Depleted uranium particles could also be found in the HEPA filter system. A Supplement Analysis for the PEIS characterizes the use of small quantities of depleted uranium as not significant.

The NIF waste streams are not expected to be significantly impacted by the use of depleted uranium. Depleted uranium shots would be expected to displace other experiments that would have generated LLW, and thereby would not create a net 
increase in LLW quantities. On a yearly basis, 5 grams of depleted uranium would be distributed through the low-level and mixed waste streams from the NIF. This quantity adheres to acceptance criteria for both the Nevada Test Site and the vendors receiving LLNL mixed waste.

\subsubsection{Methods}

To complete a PPOA, the NIF will follow one of several protocols, depending on the project phase at which the PPOA is undertaken. As discussed in Section 3.3.1, when it is operational, the NIF will follow PPOA protocols as outlined in the DOE performancebased course "Pollution Prevention Opportunity Assessment." This method is explained in more detail in Appendix A. Predictive PPOAs (those based on predictions of waste stream components identified in a design), however, follow a more abbreviated protocol. Predictive PPOAs focus on understanding the front end (the process producing the waste stream) and identifying a wide range of potential options that engineers can study as they continue with the project design. Guidelines for this abbreviated protocol are noted in Section 4.6.1.2.

\subsubsection{Procedure for Operational Waste Stream Assessment and Minimization}

The NIF has drafted an initial procedure for waste stream assessment and minimization, based on the Lasers Directorate's Nova Program. This procedure will be updated to follow more closely the DOE's PPOA methodology prior to the NIF startup.

Using a procedure similar to this for the characterization of NIF waste streams and for the analysis of P2 opportunities, the NIF operational waste streams will be periodically evaluated and ranked in order of greatest concern. Once prioritized, the P2 opportunities for the prioritized waste streams are evaluated for cost and benefits.

\subsubsection{Predictive PPOAs}

The initial PPOAs for predicted NIF waste streams involve the following steps.

1. Identifying and describing the processes and parameters responsible for the waste stream.

2. Identifying with as much specificity as possible the components that make up the waste stream.

3. Identifying and discussing P2 options that either have been, will be, or could be implemented.

\section{Step 1: Process Description}

All of the processes responsible for contributing to a waste stream under consideration (identified in the PEIS) are classified and described to the extent possible. Descriptions may come from the CDR documents, other more recent design documents (e.g., Title I or II design review documents), or discussions with the systems engineers 
responsible for the processes. Verbal descriptions are adequate, although drawings or flow diagrams should be included if they exist.

\section{Step 2: Identification of Waste Stream Components}

Waste stream components from the processes described in Step 1, above, should be enumerated to the extent possible. Such identification should, if possible, include a brief reference describing how the wastes are generated. In each case, the waste types of the components (e.g., hazardous liquid or solid low-level) are described.

\section{Step 3: Development and Description of Waste Reduction Options Examined}

First, the following types of changes are evaluated to generate options that could reduce the anticipated waste streams.

- Material changes (e.g., substitution of materials that will become less activated than those currently planned).

- Operational changes (e.g., maintenance performed on an "as needed" basis rather than on a schedule).

- Substitution of technologies (e.g., $\mathrm{CO}_{2}$ cleaning for hazardous liquid cleaning).

- Process changes (e.g., long dragout times for parts cleaned in baths).

Waste reduction options may be technical, procedural, or administrative, and they should not be limited by lack of detail (at this point). As designs become finalized, options can then be reconsidered and evaluated (when sufficient data are available) for estimated cost, technical and economic feasibility, anticipated savings, ROI, or anticipated reduction of a specific waste stream.

Finally, in cases where waste reduction options have already been considered or implemented, the PPOA documents the course of action already taken.

\subsubsection{Predicted Waste Streams and PPOAs}

The highest-priority waste streams for predictive PPOAs are those identified in PEIS Table I.4.1.2.8.1-2 (DOE/EIS-0236, September 1996). This table, which is reprinted on the following page, is an excerpt from the final PEIS. For a thorough understanding of the table, refer to the PEIS. Predictive PPOAs were conducted per Section 4.6.1.2 protocols for the waste streams identified in the PEIS table. These PPOAs are discussed in more detail in Section 5.0, and their presentation follows the same sequence of wastes as that given in Table I.4.1.2.8.1-2. 


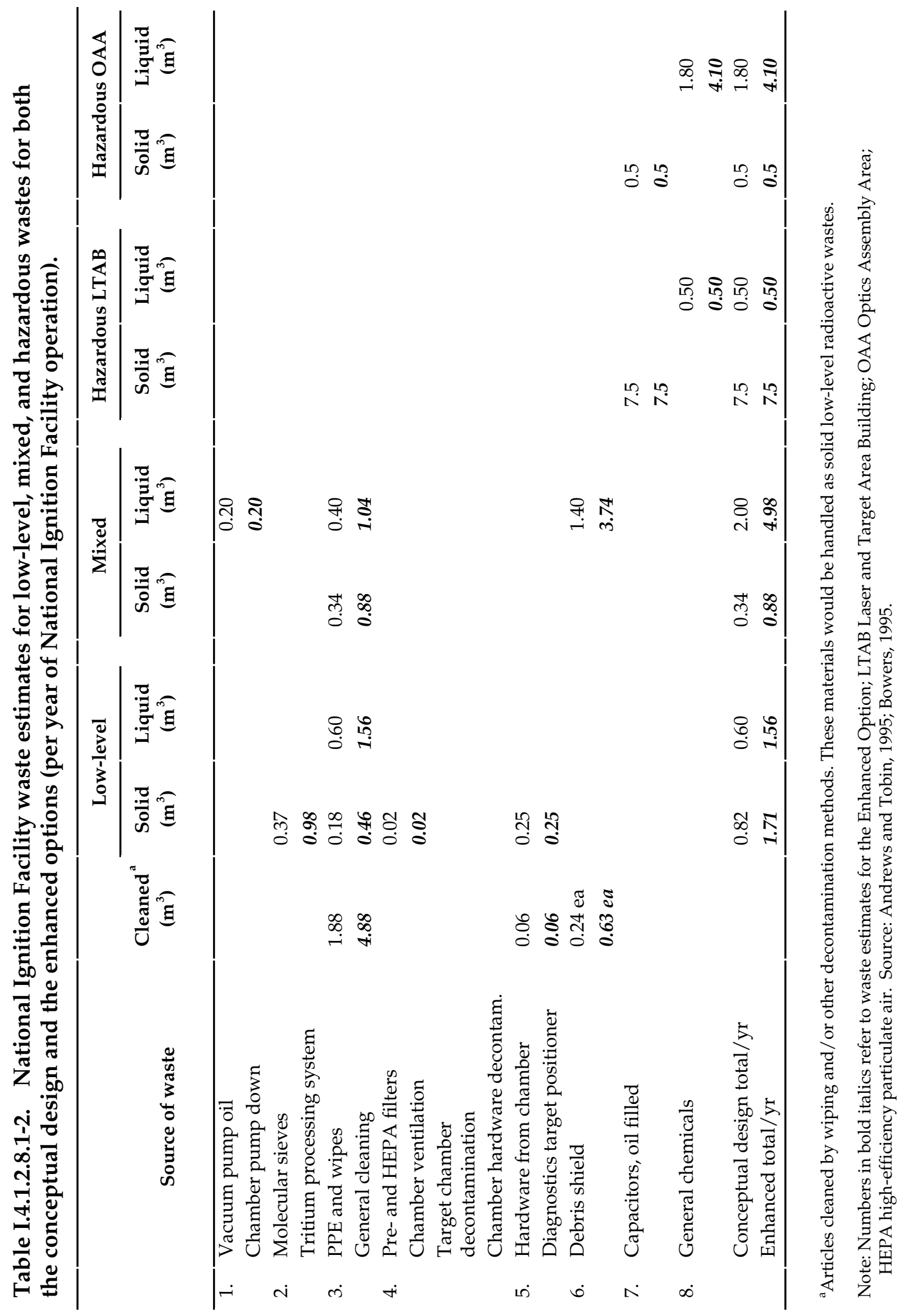




\subsection{Predictive PPOAs for NIF Waste Streams}

This section of the P2/WMin Plan includes preliminary characterizations in the form of predictive PPOAs for the waste streams identified in Table I.4.1.2.8.1-2 of Section 4.0. The numbers of and boldface-type titles for these predictive PPOAs correspond to likeidentified entries in the PEIS Table. The PPOAs are numbered and named as follows:

1. Vacuum pump oil.

2. Molecular sieves.

3. Personal protective equipment.

4.A. Pre- and HEPA filters.

4.B. Target chamber decontamination.

5. Hardware from the chamber.

6. Debris shield cleaning.

7. Capacitors, oil-filled.

8.A. General chemicals.

8.B. General chemicals, optical component processing, B391.

\subsection{PPOA 1: Vacuum Pump Oil}

According to the PEIS, vacuum pump oil and chamber pump-down were expected to generate mixed liquid waste. This waste stream, as described in the PEIS, originated primarily from the roots blower assemblies for the target chamber. Oil-free pumps have been evaluated with respect to cost and reliability for NIF applications. Oil-free pumps are considered to be acceptable for program use, eliminating this waste stream.

\subsubsection{PEIS Item 1: Vacuum Pump Oil WBS 1.8.1.5}

\subsubsection{Process Description}

The target chamber is a passive component that provides structural mounting for the final optics assembly (FOA), diagnostics, first-wall shielding, and vacuum system. It is an integral part of the vacuum-containment system. The target chamber consists of a hollow, spherical vacuum chamber with vacuum-tight ports to support the final optics, diagnostics, and target alignment/positioner systems. The sphere is mounted on a hollow-pedestal support approximately centered in the target area building. The target chamber vacuum system will provide the mechanism to evacuate the chamber and maintain its vacuum during a test sequence. 


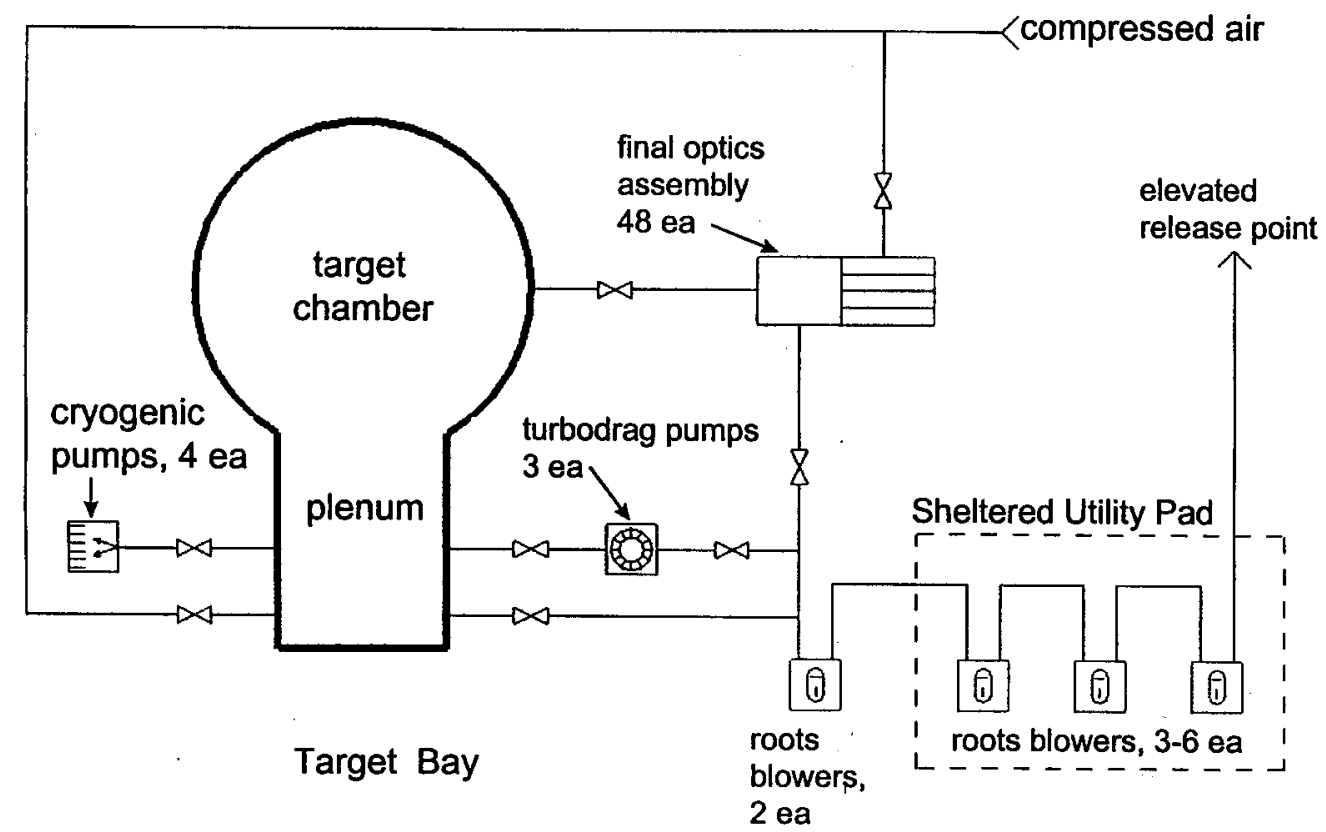

Figure PPOA 1.1. Simplified schematic of the vacuum system for the NIF target chamber. All pumps associated with this system are required to be oil-free. Source: NIF presentation NIF-0005490-10.

The target area vacuum system (Figure PPOA 1.1) is designed with adequate rough pumping capacity to simultaneously pump all diagnostics, as well as the target chamber, and to exhaust directly to a tritium cleanup system. Connecting the diagnostic vacuum systems into the target chamber system provides single-point control of tritium from all vacuum systems. All high-vacuum pumps will be of the capture variety, so that additional means of processing tritium exhaust gas will not be required. The highvacuum pumps will be valved off from the diagnostics when regenerating, so the captured gas, when released from the high-vacuum pumps, will be pumped out directly by the roughing system for processing, or by a separate system for direct tritium control. All vacuum systems are required to be oil-free to prevent possible oil vapor contamination of diagnostic and laser optics and sensitive diagnostic hardware.

\subsubsection{Identification of Waste Stream Components}

The Subsystem Design Requirements document for the Target Chamber Subsystem (SSDR 1.8.1) specifies that vacuum pumps and components must prevent the backstreaming of oil into the target chamber. This requirement is being met by the further requirement that all pumps are oil-free. As demonstrated by this requirement for oil-free vacuum pumps, there will no longer be a vacuum pump oil waste stream from NIF target chamber operations. 
This waste stream, as originally envisioned, would have contained tritiated vacuum pump oil from the chamber and from maintenance activities. Similar systems (e.g., those used in Nova and Beamlet) that currently use vacuum pumps with oil generate mixed waste streams from shots and maintenance activities.

\subsubsection{Development of Waste Reduction Options}

The primary P2 option for this PEIS-identified waste stream was implementation of oil-free pumps for all target chamber operations. In the time since the PEIS was developed, NIF project engineers examined oil-free vacuum pumps, and they moved to require that all vacuum systems be oil free. The oil-free pump option eliminated this waste stream.

\subsection{PPOA 2: Molecular Sieves}

Molecular sieves and the tritium processing system (TPS) are expected to generate solid LLW. The TPS will manage tritium from the target chamber and other components with the potential for tritium contamination (e.g., vacuum and diagnostic systems associated with the target chamber), LTAB decontamination workstations, and the target receiving and storage station.

\subsubsection{PEIS Item 2: Molecular Sieves Tritium Processing System (TPS) Description WBS 1.8.5.1}

\subsubsection{Process Description}

This PPOA examines the design of the proposed NIF tritium processing system (TPS). Life-cycle materials, P2/WMin concerns, and energy costs were included as criteria in the design process. The current design, therefore, is being optimized for P2/WMin from the standpoint of project cost. This PPOA documents the P2/WMin features incorporated into the NIF TPS.

The proposed NIF TPS design is based on the operationally proven tritium processing system that was designed and installed in the LLNL Tritium Processing Facility (Building 331) in 1992. This system has an excellent five-year performance record and represents optimal current technology in tritium-removal efficiency, energy use, and waste stream volume. The function of major components in the existing system at the LLNL Tritium Processing Facility and the new NIF designs are essentially the same. Minor design changes were required to address the specific mass, volume, and pressure requirements associated with evacuation of tritium from the NIF target chamber. In addition, the system may use a recirculating process rather than a singlepass mode. The new design will use fewer components, will be easier to operate, and will cost less to fabricate. Because these design changes are extremely preliminary, the following sections provide a brief description of the form and function of the baseline NIF TPS design. 
The NIF TPS will be located in the Laser and Target Area Building (LTAB) decontamination room. The system will convert tritium present in these systems into tritiated water via catalytic conversion. The tritiated water will be collected and stored in dryer beds.

\subsubsection{Equipment Description}

The TPS will consist of skid-mounted process hardware and remotely located monitoring control systems. Primary tritium-processing hardware will include the following components (see Figure PPOA 2.1):

- A gas-expansion tank to provide storage volume for contaminated gas liberated from the target chamber vacuum system during the regeneration cycle of the cryopumps.

- Hermetically sealed blowers and piping to carry gases from tritium-exposed systems to the TPS.

- Two TPS modules, each consisting of the following major components (see the TPS module schematic in Figure PPOA 2.2):

- Heat exchanger to preheat and cool gases during the cleanup cycle.

- Preheater reactor to provide catalytic conversion of tritiated gases to tritiated water.

- Dryer beds that contain approximately $100 \mathrm{~kg}$ of molecular sieve material (synthetic zeolite desiccant) for removal of tritiated water from the gas stream.

The TPS monitoring features allow for real-time gas flow rates, system pressure and temperature readings, and system inlet/outlet contamination levels. The TPS controls integrate monitoring data and provide for preheater and reactor temperature control. 


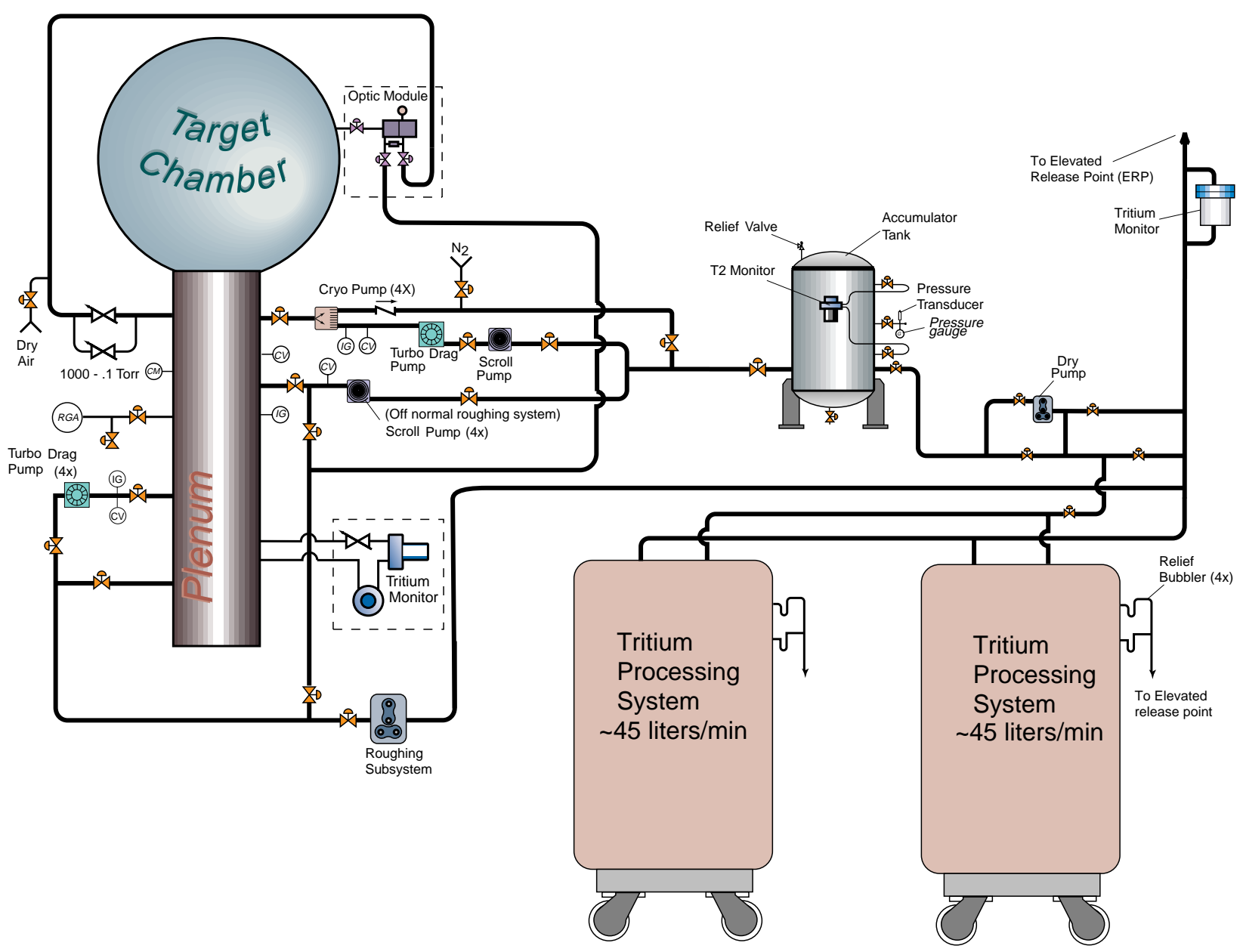

Figure PPOA 2.1. Tritium processing system layout. Source: T. Reitz, P. Dohoney, F. Drobnik, M. Evans, B. Fischer, S. Holmes, F. Lopez, and Gil Wong. Environmental Protection Systems, WBS 1.8.5 Title I Presentation. LLNL, November 13, 1996. 


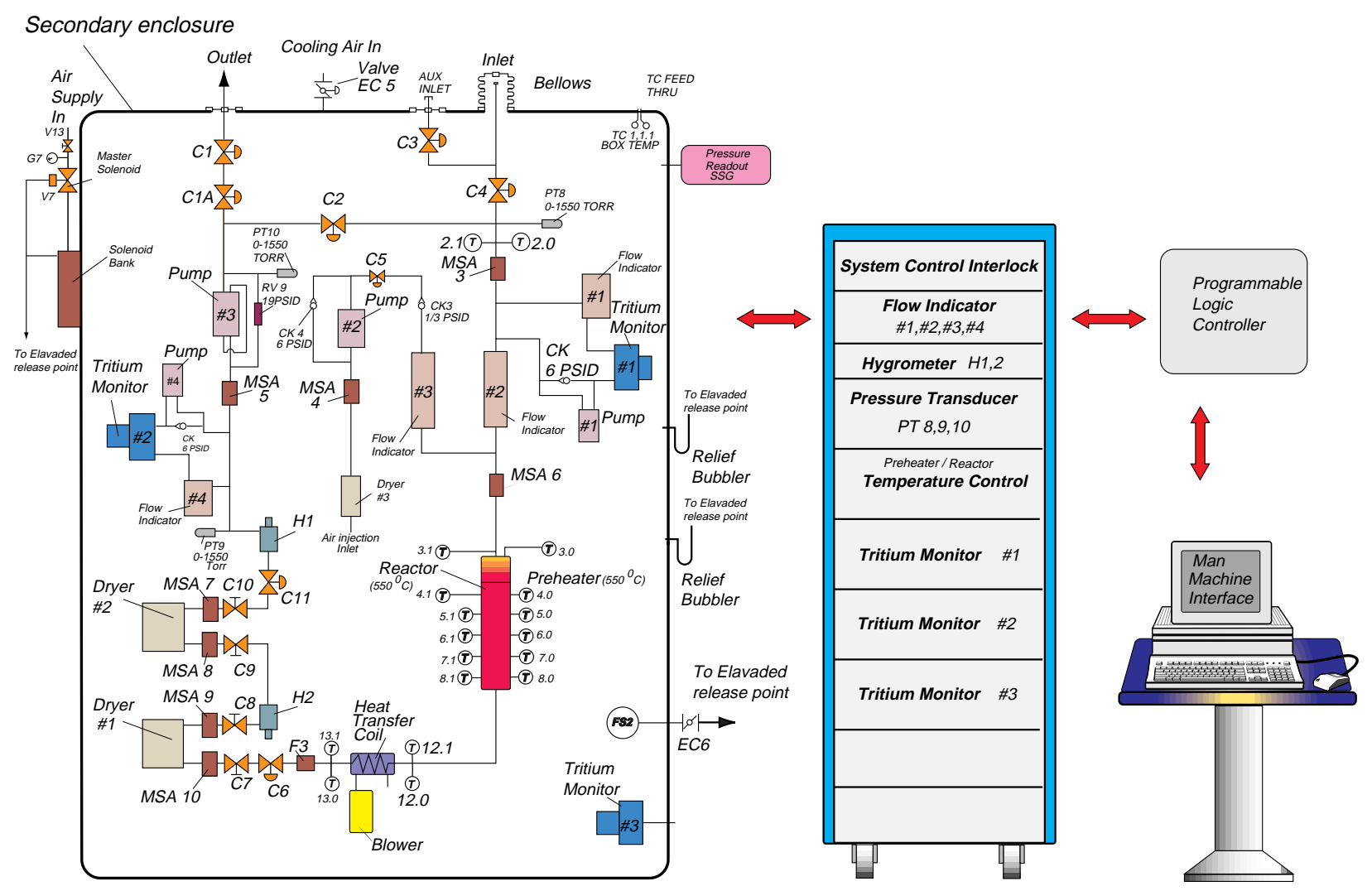

Figure PPOA 2.2. Tritium processing system module. Source: T. Reitz, P. Dohoney, F. Drobnik, M. Evans, B. Fischer, S. Holmes, F. Lopez, and Gil Wong. Environmental Protection Systems, WBS 1.8.5 Title I Presentation. LLNL, November 13, 1996.

\subsubsection{Tritium Processing Cycle}

Before a NIF laser shot, the target chamber will be pumped down to a vacuum pressure of $10^{-5}$ Torr. Approximately $99.5 \%$ of the tritium from the chamber, plenum, and associated hardware will be captured on the cryopumps. The cryopump isolation valve will then close, preventing gases from returning to the plenum and chamber via the cryopump. The cycle will be repeated after every shot. After approximately six weeks (under direct-drive conditions), the cryopumps will be regenerated. This regeneration procedure removes tritium from the cryopumps and runs the tritium gas through the TPS to generate tritiated water and contaminated canisters.

Under direct-drive conditions (the most intense shot schedule), the NIF will manage targets containing up to 1,750 $\mathrm{Ci}$ of tritium per year. However, the total amount of tritium on site at any one time for the NIF is $500 \mathrm{Ci}$, which is distributed as follows:

- $200 \mathrm{Ci}$ in the cryopumps.

- $200 \mathrm{Ci}$ on the TPS dryer beds.

- $75 \mathrm{Ci}$ in the target storage and handling area. 
- $25 \mathrm{Ci}$ distributed on the first wall, target chamber, pumps, HEPA filters, and similar surfaces.

The regeneration of the cryopumps and disposal of the molecular sieve canisters will be driven by the NIF facility's tritium budget, as follows:

- Maximum tritium accumulated per week $=(1,750 \mathrm{Ci}) \div(52$ weeks $)=34 \mathrm{Ci} /$ week.

- Regeneration time $=(200 \mathrm{Ci}) \div(34 \mathrm{Ci} /$ week $)=6$ weeks .

During the regeneration process, cryosorbed and cryocondensed materials will sublimate and be drawn into a 10,000-L gas expansion tank. Gases will then be processed through the TPS at a rate of $90 \mathrm{~L} /$ minute $(45 \mathrm{~L} /$ minute for each of the two TPS modules). Gases will enter a TPS module through the catalytic preheater, where tritium will be oxidized to form tritiated water. This water will be vaporized, then recovered from the gas stream as it passes through the dryer bed canisters. Although all the tritiated water should be removed by the first canister, a second canister will be placed in series with the first as a safety measure. The remaining gas stream will be monitored for tritium before being exhausted to the outside.

\subsubsection{TPS Waste Streams}

Under direct-drive conditions, it is expected that the first canister of each TPS module will be changed every 12 weeks (after two cryopump regenerations), generating 9 to 10 canisters per year.

At the time of disposal, each canister will have two valves attached (Valves C7 and C8). Whereas these valves could, in theory, be reused, they form an easy and safe way to detach the canisters, minimizing personnel exposure to tritium. The canisters will be drummed (Type A container) and disposed of as a routine, low-level, certified waste destined for the Nevada Test Site.

Components of other waste streams from routine operation and maintenance of the TPS are:

- Preheater reactors. The preheater reactor replacement period is anticipated to be every 10 years. Over the 30 -year life span of the NIF, each reactor in the TPS modules will changed three times. They will be disposed of as low-level waste.

- Gloves on glove box. Gloves will be replaced every six months.

- Metal bellows pumps. Within each TPS unit are four metal bellows pumps. Each metal bellows pump will be changed once, approximately halfway through the NIF's anticipated period of use. Two sets of metal bellows pumps will be used during the NIF life cycle.

\subsubsection{P2 in Design of Process}

All pumps are dry, eliminating the generation of tritiated oil, a mixed waste. Duplication of existing equipment provides a reliable system at reduced cost. 


\subsection{PPOA 3: Personal Protective Equipment}

Personal protective equipment (PPE) and wipes, along with general cleaning, are expected to account for both solid and liquid LLW. This is a diverse waste stream that will be generated in many different areas of the NIF. Most maintenance procedures have not been formalized yet, making a thorough understanding of PPE and wipes waste streams difficult. This PPOA considers only the PPE waste stream. A discussion of wipes is incorporated into PPOA $8 \mathrm{~A}$ and $8 \mathrm{~B}$.

\subsubsection{PEIS Item 3: Personal Protective Equipment WBS 1.8.5}

\subsubsection{Process Description}

Radiologic worker protection requirements will mandate the wearing of PPE when individuals work inside the target chamber or with contaminated hardware. Several activities may contribute to the generation rate of tritium-contaminated PPE. Examples of such activities include cleaning of the target chamber itself, cleaning of target chamber insertions, debris-shield removal, and cleaning and replacement of damaged diagnostic hardware.

Detailed operations planning for the NIF is not yet underway. Laboratory ES\&H policy states that PPE is not a substitute for adequate engineering or controls, which are always the first levels of protection from hazards. The NIF is actively developing operations concepts that minimize the need for manual cleaning and target chamber entry.

\subsubsection{Identification of Waste Stream Components}

PPE from NIF operations will be generated by activities associated with worker exposures to tritium contamination inside the target chamber and from handling contaminated hardware. It should be noted that PPE waste may also be generated from clean room activities in the OAB (see PPOA 8B), but this PPE will not be tritiumcontaminated and will be launderable and reusable.

PPE is currently expected to be placed in specially designed containers after each use. Options for final disposition include disposal as LLW and laundering and reusing PPE as long as it meets safety requirements.

\subsubsection{Development of Waste Reduction Options}

Launderable PPE is currently being used in the Laser Program's Nova facility. Such PPE is also in use in various programs throughout the DOE complex and represents the best P2 option for this waste stream.

Two other P2 options merit a brief discussion. They are:

- Minimizing generation by using robotic operations in the chamber. 
- Carefully keeping LLW-contaminated PPE segregated from other PPE and wipes materials. This would include ensuring that contaminated PPE laundry containers are protected from rain and other conditions that could result in the spread of contamination.

The NIF design includes the development of robotic capabilities for the target chamber that may be adaptable to target chamber cleaning tasks, once the operational planning for these tasks is complete and they are better understood.

Launderable PPE is currently used at Sandia National Laboratories/New Mexico for Environmental Restoration field activities and the Moly (99) Project involving radiological operations. The use of PPE associated with the Moly (99) Project involves the production of medical radioisotopes to treat and diagnose patients with cancer.

In this case, reusable (launderable) PPE was purchased using DOE ROI funds to reduce the LLW generation. Researchers estimated that one reusable garment was required for every 100 disposal garments. The result was a significant reduction in the quantity of LLW generation by operations requiring PPE use. It is also notable in this case that workers felt that the launderable garments were significantly lighter and cooler than disposables.

\subsection{PPOA 4A: Pre- and HEPA Filters}

Prefilters and high-efficiency particulate air (HEPA) filters for chamber ventilation, target chamber decontamination, and chamber hardware decontamination are expected to contribute solid LLW. The replacement schedules for both pre- and HEPA filters are driven by health and safety standards.

\subsubsection{PEIS Item 4: Pre- and HEPA Filters WBS 1.2.2/1.8.5}

\subsubsection{Process Description}

HEPA filters within the NIF will provide filtration for two discrete applications: clean rooms and the reduction of emissions. Air-cleaning devices reduce contaminants from effluents to below allowable limits before being discharged to the environment. Filters are normally employed to remove particulates, and carbon adsorbent is used to remove gases and vapors. The most efficient air cleaner for particulates is a HEPA filter. Other cleaning devices, such as electrostatic precipitators, cyclones, scrubbers, incinerators, and dust filters, may be used depending on the type, nature, and concentration of contaminants in the effluent. LLNL Hazards Control determines the proper application of HEPA filters and other air-cleaning devices. The EPD determines the proper application of air-cleaning devices to meet BAAQMD and NESHAP requirements. 
The NIF was characterized in the PSAR as a low-hazard radiological facility. DOE has concurred in this hazards category. This type of facility is required by the DOE, the EPA, and LLNL to contain any possible radiologic contamination with a single HEPA-filtered HVAC system. These designs are governed by the LLNL ESEH Manual, which requires that HEPA filters pass acceptance tests and undergo periodic performance tests.

The entire laser system will be slightly pressurized with a clean gas to prevent particle deposition on critical optical components. The target bay will have Class 100,000 HEPA-filtered airflow to the pole regions above and below the target chamber. The target bay HVAC system will provide a slight negative pressure in the room at shot time with exhaust released at an elevated point to accommodate the possibility of air activation during high-yield shots. The negative pressure will be maintained by a system of exhaust fans. Exhaust air will be filtered prior to release. Fans serving the target area will be isolated from the fan room by a partition wall to provide a minimum of 2-hour fire protection and physical separation of air systems that might contain activated particles. Fans in the fan room will cause the target area to become negatively pressurized with respect to surrounding areas just prior to and after a high-yield experiment.

Operational experience with high-power laser systems demonstrates that the environment inside the laser cavity and in the vicinity of the optical components must be better than Class 1, as defined by FED-STD 209E. This level of cleanliness will be achieved using a three-level approach to achieving the cleanliness requirement, which is more economical than previous methods. This approach incorporates an air-handling system using $90 \%$ bag filters that will produce an equivalent air cleanliness of between 10,000 and 100,000 using FED-STD 209E. The filtration level will provide a barrier to the outside environment sufficient to allow the secondary and tertiary filter systems to achieve the required particulate level. The second level of filtration is provided by clean modules or zones in the areas of concern. The modules will be used during installation and maintenance. A third level of particle elimination is accomplished by enclosing the entire laser and slightly pressurizing it with clean gas (approximately $2.54-$ to $10.2-\mathrm{cm}$ water column). It is necessary to provide clean nonreactive gas to the laser system, so this gas system and enclosure is not an additional requirement.

The HVAC system in the target bay is a separate system capable of achieving a Class 100,000 clean room (LTAB Conventional Facilities Energy Conservation Report, NIF0004309, WBS 1.2.1) in areas above and below the target chamber where protection of the optical components from particle contamination is critical. This cleanliness level is achieved by the use of HEPA filters and increased air flow. Because of the potential for activated air during high-yield experiments, the HVAC system is required to be isolated, self-contained, and capable of providing a negative pressure in the target bay and exhaust release at an elevated point.

\subsubsection{Identification of Waste Stream Components}

The HEPA filter waste stream expected from the NIF during its operational phase will consist of both large and small HEPA and pre-filters. Larger filters will result from 
pre- and HEPA filters for chamber ventilation, target chamber decontamination, and chamber hardware decontamination. Such filters will account for solid LLW. Smaller HEPA filters will be installed in numerous small systems within sections of the LTAB and $O A B$ that require low contamination and/or low emissions. These areas could include smaller facilities within the decontamination area of the LTAB, such as gloveboxes, the general decontamination workstation, and low-contamination facilities for moving and storing optical components (carts and elevators). Such small systems may or may not result in LLW.

An analysis of construction work packages yields the following estimates for HEPA filters from the LTAB and OAB: approximately 225 HEPA filters will be installed in the HVAC systems along with approximately 75 pre-filters.

The systems that will employ smaller HEPA filters (such as the general decontamination workstation, and optics and component transfer points utilizing carts) have not yet been sufficiently designed to allow for an assessment of the possible numbers and sizes of filters. Although the large HEPA filters installed in the ceiling may never be changed, these smaller filters may contribute significantly to this waste stream if large numbers of changeouts are required in the systems.

\subsubsection{Development of Waste Reduction Options}

Under some circumstances, it may be possible to crush or shred HEPA filters, possibly resulting in significant volume reduction. Such treatment, however, could yield airborne activated particulates, which can cause significant worker risk and high costs to create a safe work space.

Idaho National Engineering and Environmental Laboratory has funded an investigation of the concept of washable metal HEPA filters. The prototype embodies a washable, recyclable structure with replaceable filtration components. This research was conducted at LLNL by Werner Bergman, and his paper (UCRL-ID-125048) detailing the requirements for such a system is listed in Section 7.0. The major design problem with the system was its considerable weight. Development of a cleanable HEPA filter would require significant and additional research and development funding to yield a structure acceptable for the NIF.

Another possibility for HEPA filter disposal is to carefully characterize each filter as it is removed. This approach eliminates the risk of mischaracterization, and it can possibly reduce the need for sampling and the number of filters characterized as lowlevel waste. A good reference for the level of documentation that could be required to help a characterization program is the Pacific Northwest Laboratory "HEPA Filter Disposal Checklist," which is referenced in Section 7.0.

For nuclear facility D\&D, the DOE Energy Technology Engineering Center (ETEC) has pilot tested the Torit self-cleaning filter to capture large particulate matter prior to HEPA filtration in contained work areas. The system uses a series of six prefilters in a collection drum, rather than disposable filters that must be changed frequently. 
Pulsating air is used to dislodge trapped particles from the prefilters, and the particles are collected in the bottom of the drum. Use of the new filters resulted in significant secondary waste avoidance. Such a system should be evaluated from a cost/benefit perspective and considered for use when significant amounts of activated particles are expected.

\subsection{PPOA 4B: Target Chamber Decontamination}

Target chamber decontamination and chamber hardware decontamination are expected to contribute solid LLW. First-wall design and cleaning methods have not yet been finalized.

\subsubsection{PEIS Item 4: Target Chamber Decontamination WBS 1.8.5.3}

\subsubsection{Process Description}

The target chamber is the environmental and structural boundary between the LTAB and the target. This chamber provides support for the FOA, maintains a vacuum environment for the target, and confines target debris following a shot. The chamber is a 10-cm-thick, spherical aluminum shell with a 10-m internal diameter. If the shell were subjected to the x-ray fluence associated with every shot, small portions of the aluminum wall would vaporize. In addition to resultant damage to the chamber wall over time, the vaporized material would condense on the debris shields and other chamber hardware, increasing the need for maintenance cleaning. Hence, a target chamber "first wall" will be installed. A significant portion of target chamber decontamination will occur during the cleaning and maintenance of these panels.

The expected design of the first wall and the strategies for cleaning it have changed since the Title I 65\% design review. The latest design option and associated cleaning methods are discussed here. The design and cleaning options favored at the time of the Title I 65\% design review are discussed in Section 5.5.1.3, below.

\section{Louvered First-Wall Panels}

The latest design for the first wall consists of approximately 400 stainless-steel panels measuring $1 \mathrm{~m}$ by $1 \mathrm{~m}$. Each panel has multiple "dog-legged" louvers extending from its surface (see Figure PPOA 4B.1 ). This design serves both to capture target debris when it is initially generated (including tritium and gold, copper, aluminum, beryllium, and depleted uranium "splats"), and to act as a sink when the splats are ablated during subsequent shots (see Figure PPOA 4B.2). The panels also serve as beam dumps (surfaces that absorb laser energy passing beyond the target during a shot). 


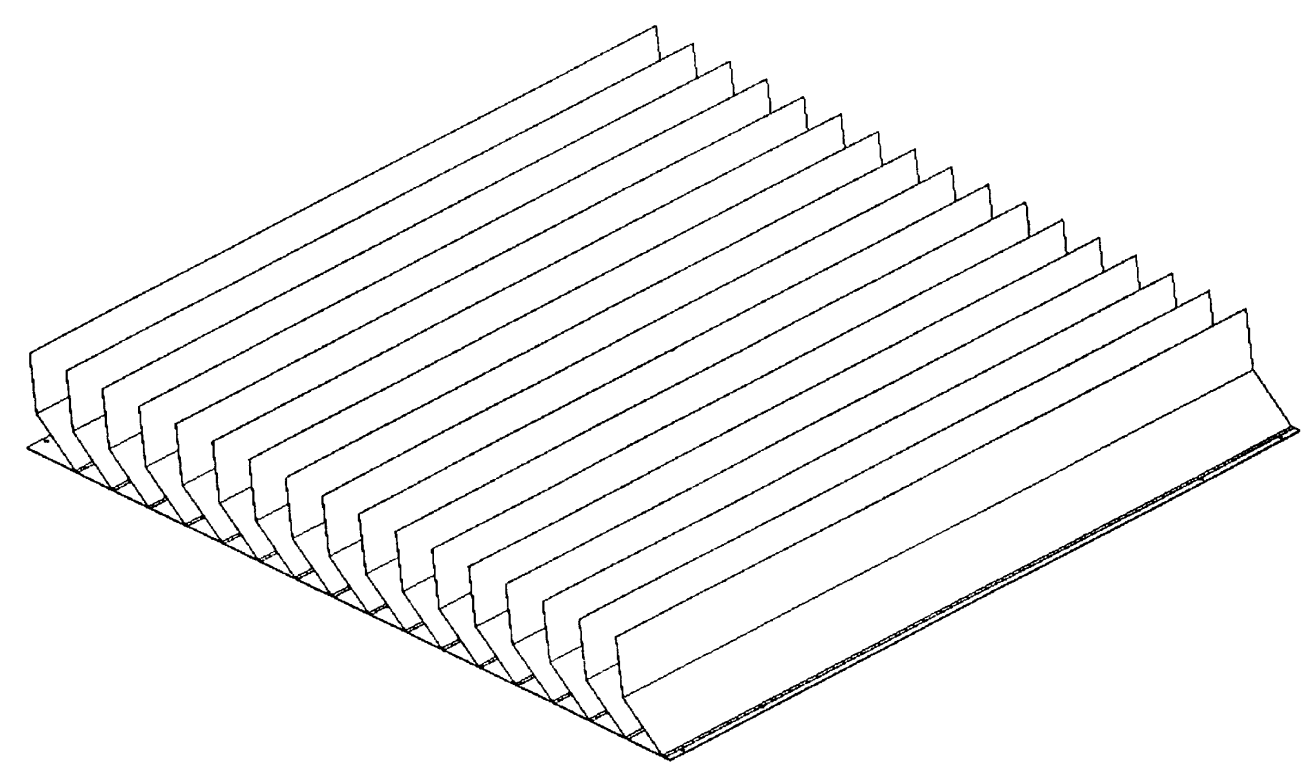

Figure PPOA 4B.1. Louvered first-wall panel. Source: W. J. Hibbard, Target Chamber First Wall Mechanical Design Workshop presentation. LLNL, March 19, 1998.

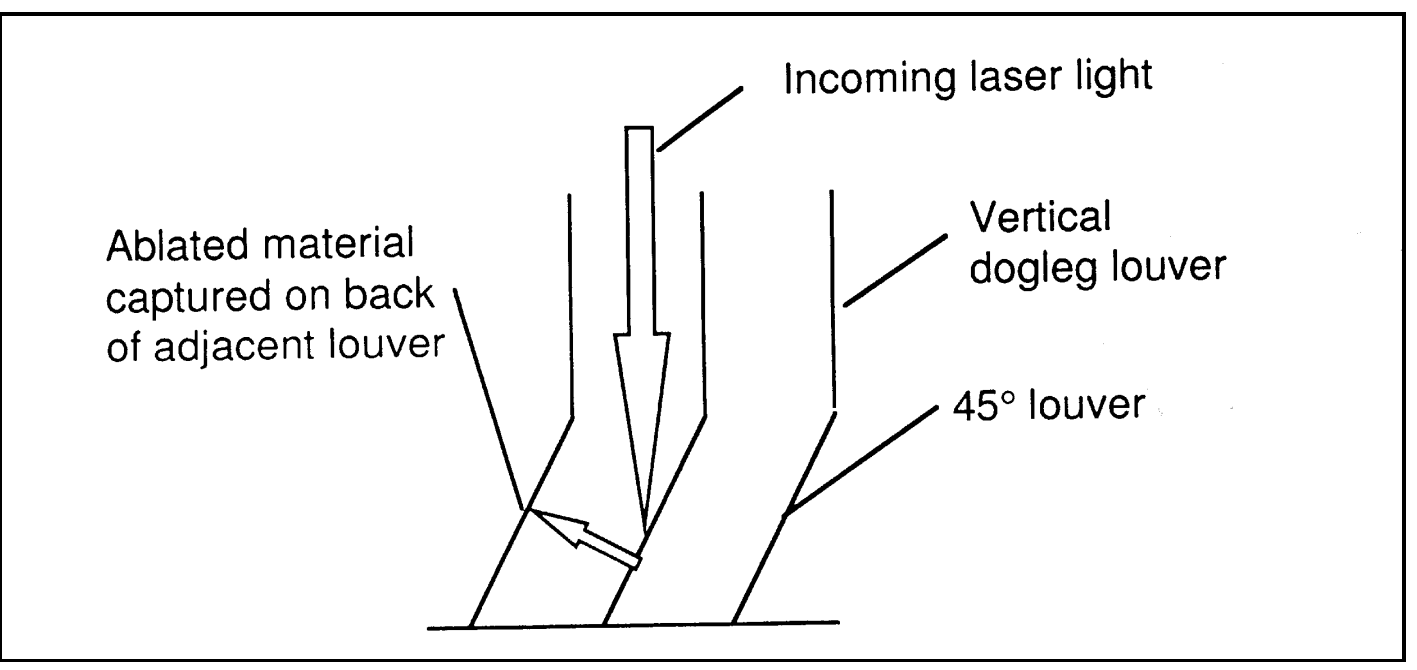

Figure PPOA 4B.2. Louvered first-wall panel edge view. Source: W. J. Hibbard, Target Chamber First Wall Mechanical Design Workshop. LLNL, March 19, 1998. 
The louvered panels are expected to weigh approximately $25 \mathrm{~kg}$ each, but their final weight depends on the thickness of the stainless-steel louvers. For planning purposes, it is currently assumed that the panels will be cleaned twice a year. Removal of particulates and hydrocarbon films will be accomplished by sending the panels through an ultrasonic wash tank, using commercially available detergent, and dryer. Metal splats are not expected to be removed by this process.

\section{Other Target Chamber Decontamination}

From time to time, when first-wall panels are removed, the target chamber itself will be wiped with a substance such as Radiac wash followed by a deionized water wipe. Areas contaminated with grease will be wiped with ethanol. Waste from the wipecleaning procedure may be disposed of as solid hazardous or mixed waste. It may be possible to develop cleaning procedures that will produce only LLW by using wipes to dryness and characterizing the wipes for metals picked up during cleaning.

\subsubsection{Identification of Waste Stream Components}

Disposal of Louvered Panels

The louvered panels are expected to possess a 10-year life span, after which time they will be changed out, generating solid LLW.

\section{Louvered Panel Cleaning}

Cleaning the louvered panels may generate low-level solid waste, but not mixed, aqueous waste.

\subsubsection{Development of Waste Reduction Options}

\section{Initial First-Wall Design}

The composition of the target chamber first wall was originally envisioned as 350 boron-carbide-coated aluminum panels, each approximately 1-m square. The panels would be cleaned periodically to prevent remobilization of debris onto the debris shields. The panels were designed to be manipulated robotically for removal, cleaning, and reinstallation. Much effort went into investigating methods for cleaning the first wall, including $\mathrm{CO}_{2}$ pellet cleaning, bead blasting, and laser cleaning, to minimize the waste stream for the cleaning of the aluminum panels. $\mathrm{CO}_{2}$ pellet blasting was the initial baseline process, and the bead blast cleaning process was favored as the baseline at the time of the Title I 65\% design review. 
$\mathrm{CO}_{2}$ Pellet Blasting

The initial option considered for cleaning the aluminum first-wall panels involved remote decontamination. A robotic arm inserted into the chamber would carry a $\mathrm{CO}_{2}$ pellet blasting device and a recovery system to capture the particulates liberated from the panels. This method minimized the need for personnel entry into the target chamber and generated a minimal quantity of waste.

Initial tests of the process cleaning efficiency for the panels, using a surrogate vapordeposited copper contaminant, were promising (82\% removal efficiency after one pass). However, Nova-generated copper and gold splats were more difficult to remove (30\% removal efficiency after three passes). With the apparent need for highly aggressive pellet cleaning parameters, several negative aspects of in situ $\mathrm{CO}_{2}$ operations were revealed. The primary concern with the use of large quantities of $\mathrm{CO}_{2}$ pellets $(\sim 363 \mathrm{~kg} / \mathrm{hr})$ on the target chamber was that they would cause significant temperaturecontrol difficulties in the target chamber and building for several days after cleaning. The building's thermal stability would also be adversely impacted by the large exhaust system required to capture the $\mathrm{CO}_{2}, \mathrm{~N}_{2}$, and debris. Such factors resulted in the selection of out-of-chamber bead blasting as the baseline at the time of the Title I 65\% design review.

\section{Bead Blasting}

With the bead-blasting method, first-wall panels would be removed by the target chamber robot, then transferred to an enclosed bead-blasting $/ \mathrm{CO}_{2}$ cleaning line. The first-wall panel cleaning station, located in the target chamber decontamination area, would be similar to a large glove box and would contain a glass bead nozzle, $\mathrm{CO}_{2}$ dusting nozzle, and an inspection station. The system could process 50 panels/week during a weekly 16-hour maintenance schedule. Following the cleaning, the panels would then be reinstalled robotically.

The need to remove first-wall panels from the chamber required consideration of workers' exposure to gamma radiation. Several options were considered for minimizing worker exposure during the transport of the panels to the cleaning station, including minimization of the mass of the panel backing plates, shielding, and remote operations.

Early results suggested that bead-blast cleaning provided a $96 \%$ cleaning efficiency. Because the process requires removal of panels from the chamber, its use necessitates the presence of a robot. Unlike in situ $\mathrm{CO}_{2}$ cleaning, bead blasting imparts no thermal impact on the chamber and minimal in-chamber particulate generation. In addition, a smaller capital expenditure would be needed to establish the bead-blasting system.

\section{Laser Cleaning}

Laser cleaning was identified and considered as a possible option for the first-wall aluminum panels. Early evidence suggested that the technique removed the necessary 
debris, but it was more expensive than bead blasting. Further research and development with the vendor would have been necessary before this method could have been applied to the panels. Cost and time constraints prevented further consideration of this method.

\subsubsection{Waste Reduction Approaches}

Louver Design

Further efforts in the continuing design of first-wall panels should maximize their useful lifetime. A key parameter, the thickness of the louvers, should be optimized to reduce the weight of the panels while maintaining functionality and durability.

\section{Louver Panel Washing}

Future design efforts for the panel washing process, including development of the parts washer specifications, should be geared toward ensuring that the process does not generate mixed waste. If louvered panel washing generates a low-level waste stream, a volume-reduction step, such as evaporation, should considered to be added to the process.

\subsection{PPOA 5: Target Chamber Hardware}

Hardware from the chamber and diagnostics target positioner is expected to account for solid LLW. This waste stream represents target hardware that is no longer usable following a shot, hardware that is discarded after several cleanings, and hardware that is not practical to clean.

\subsubsection{PEIS Item 5: Target Chamber Hardware Target Positioner and Target Diagnostics WBS 1.8.2 and 1.8.3}

\subsubsection{Process Description}

Target chamber hardware for the NIF, as defined in the PEIS, includes the target positioner system and target diagnostics. The target positioner system provides for the placement, stability, and alignment of both the target and the target alignment sensor (TAS) within the target chamber. Target diagnostics will include optical, $x$-ray, and nuclear diagnostics to collect information in support of the NIF experimental plan regarding laser system performance, ignition and weapons physics, and radiation effects. 
Target Positioner System

The target positioner (see Figure PPOA 5.1) and target alignment sensor positioner (TASPOS) are manipulators with multiple degrees of freedom that allow for repeatable, remote positioning of the target and the TAS within the target chamber. The positioners will be inserted into the target chamber through opposing vacuum isolation valves.

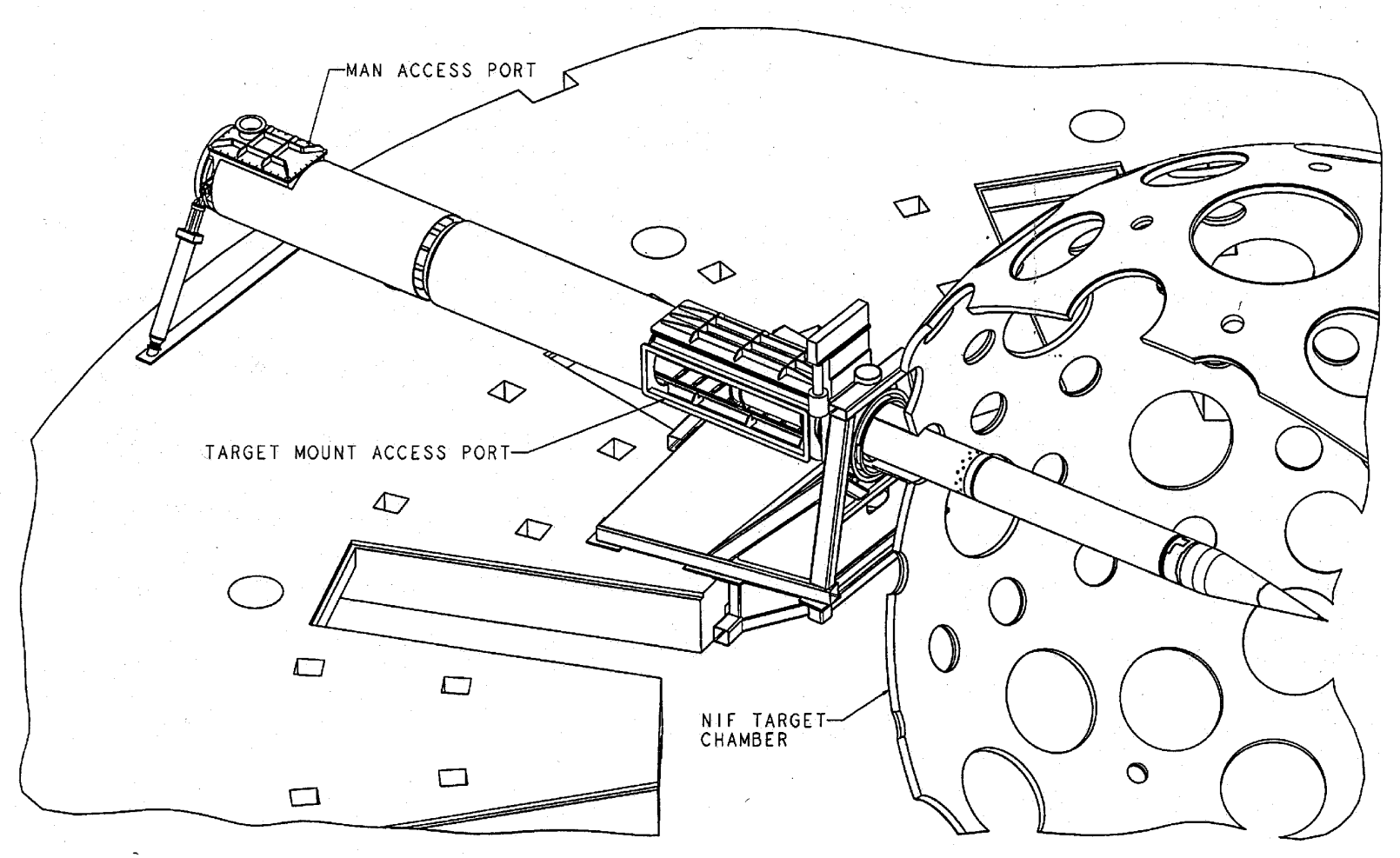

Figure PPOA 5.1. Target positioner from target insertion side. Source: NIF Target Positioner, 65\% Title II Design Review, January 1998.

The TAS is a reference point for the alignment of both the target and the laser. It will occupy the center of the chamber during the alignment phase, then the TASPOS will retract from the chamber into a protective enclosure. The target positioner, holding the target at the beam's focal point, will remain extended during a shot. The TASPOS will be nearly identical in design to the target positioner boom, discussed below.

The target positioner will be a telescoping boom made of a graphite-fiber-reinforced composite material, covered by a thin aluminum shell. These materials are low activation and are resistant to ablation and degradation from debris, $x$-ray fluence, neutrons, and the extreme thermal and vacuum conditions present in the chamber environment. The target positioner boom when fully retracted will be accessible to 
personnel outside of the chamber. Cleaning, maintenance, and installation of targets will occur with the boom in the retracted position. Although the need and frequency of cleaning of the positioners has not been fully defined, cleaning will likely occur via wiping. The end of the boom will carry the conical target assembly, containing the mechanisms for fine-scale adjustment of target position and the target, supported on a thin rod of alumina or other material. In addition, according to the NIF experimental plan, the target assembly must be capable of accommodating apparatus to maintain cryogenic targets.

During shots, portions of the rod supporting the target will be vaporized. The target assembly will be protected from $x$ rays and debris by a shock absorber consisting of aluminum foam between an ablative layer of boron carbide $\left(\mathrm{B}_{4} \mathrm{C}\right)$, and a thin aluminum plate. This shock absorber will become activated during shots with significant neutron yield, and will be disposed of as required.

\section{Target Chamber Diagnostics}

The NIF target chamber diagnostics will be brought on line as needed to meet the NIF experimental plan. Table PPOA 5.1 lists the Phase I diagnostics that will be used during start-up experiments to test the performance of the laser system and to "tune" the hohlraum. Table PPOA 5.2 lists the Phase II diagnostics, which will come on-line for cryogenic and pre-ignition experiments, and eventually, ignition shots. These tables show how many diagnostics will be used during the two phases, and they identify whether a camera is used for a particular type of diagnostic. Use of filmless chargedcoupled device (CCD) cameras reduces the photochemical waste stream associated with traditional photographic methods. 
Table PPOA 5.1. Phase I diagnostics.

\begin{tabular}{|l|c|c|}
\hline \multicolumn{1}{|c|}{ Title } & Abbreviation & Camera used \\
\hline Time-resolved x-ray imaging system & TRXI & Yes \\
\hline Soft x-ray imager & SXRI & Yes \\
\hline Static x-ray imager & SXI & Yes \\
\hline X-ray streak & SSC & Yes \\
\hline Soft x-ray power diagnostic & SXSS & No \\
\hline Shock breakout, passive & SOP & Yes \\
\hline Shock breakout, active & ASBO & Yes \\
\hline Filter flourescer & FFLEX & No \\
\hline Total neutron-yield detectors & YN & No \\
\hline Neutron time of flight & NTOF & No \\
\hline Neutron imaging system & NI & Yes \\
\hline Aftershock breakout & ASBO & Yes \\
\hline Full-aperture backscatter station & FABS & Yes \\
\hline Near-backscatter imager & NBI & Yes \\
\hline Henway diagnostic & & Yes \\
\hline
\end{tabular}

${ }^{a}$ Use of a charged-coupled device (CCD) camera may not be feasible for this diagnostic. Film will be used.

Table PPOA 5.2. Phase II diagnostics.

\begin{tabular}{|l|c|c|}
\hline \multicolumn{1}{|c|}{ Title } & Abbreviation & Camera used \\
\hline Neutron diagnostics & & \\
Neutron spectrometer & NS & No \\
Bang-time and burn duration & BTBD & Yes \\
Neutron coded-aperture microscope & NCAM & Yes \\
Gamma-ray spectrometer & GRS & No \\
X-ray diagnostics & TRXS & Yes \\
Time-resolved x-ray spectrometer & MXI & Yes \\
Monochromatic x-ray imager & SXI & Yes \\
X-ray imager & & \\
Other diagnostics & EBM & No \\
Energy-balance module & & Yes \\
SRS spectrometer & CO & Yes \\
Cassegrainian optic &
\end{tabular}

Diagnostics will extend into the chamber either independently, or they will be housed in diagnostic instrument manipulators (DIMs). To help minimize neutron 
activation of the hardware, DIMs are currently expected to be made of aluminum. Where possible, other target diagnostic hardware will be constructed from coated aluminum or other low-activation materials. Other steps that will help minimize activation will include the removal of diagnostics not needed during a shot from the chamber. Most diagnostics will not be used during high-yield shots.

Cleaning of the diagnostic equipment is expected to be accomplished by removing the diagnostic from the chamber and transporting it to the target chamber decontamination area. There, a variety of cleaning methods may be used, including wipe cleaning and $\mathrm{CO}_{2}$ snow cleaning.

\subsubsection{Identification of Waste Stream Components and Amounts}

\section{Target Positioner}

The portions of the target assembly that are vaporized at the time of a shot will be deposited on the chamber wall. This waste is accounted for in the target chamber decontamination PPOA. The aluminum foam $/ \mathrm{B}_{4} \mathrm{C}$ shields used during yield shots will be disposed of as solid LLW. Wipe cleaning of the target positioner will also generate LLW.

Target Diagnostics

The consumable parts associated with many of the diagnostics include beryllium screens and pinhole disks. These parts will be changed as needed and disposed of as LLW. Wipe cleaning of the diagnostic hardware will remove mostly particulates and will generate a LLW stream. From time to time, particularly following high-yield shots, some diagnostics will be irreparably damaged. Such hardware will be disposed of as LLW.

\subsubsection{Development of Waste Reduction Options}

Target Diagnostics

As the design efforts for target diagnostics continue, every effort should be made to construct them out of low-activation materials. The surfaces of equipment should be smooth with fairly simple surface geometries to facilitate cleaning.

\section{Beryllium Filters}

The beryllium filters and windows on diagnostics will be damaged at the time of a shot by splats and ablation, and they cannot generally be reused. These items are approximately $99.8 \%$ pure before use, and they are fairly expensive to purchase. It 
would be worth investigating whether the items could be sold back to the vendor as a less-pure beryllium source.

\section{Cryogenic Target Positioner (Cryostat)}

The function of the cryogenic target positioner, currently in design, will be to maintain deuterium-tritium targets in a frozen state for up to several days, in preparation for a high-yield fusion shot. The cryogenic positioner, or cryostat, will fit on the end of the target positioner boom. Essential components to the cryostat include a general support structure, pressure tanks, and tubing for cryogenic liquids. The apparatus will weigh up to $200 \mathrm{~kg}$.

Although this apparatus will not be used in the NIF until approximately the year 2005, various design options are currently being considered to minimize its neutron activation (Latkowski and Sanchez, in press). The ultimate motivations for consideration of cryostat design are the need to reduce the amounts of energy deposited in the cryogenic fluids, and worker radiological dose. The decisions, however, will have a P2 impact by minimizing quantities of activated waste generated by cryogenic shots.

The baseline cryostat design uses stainless-steel structural components and aluminum tanks. This composition would become activated following a shot, resulting in unacceptable dose levels to workers. A nose cone made of copper was proposed as a baseline for a shielding device. Although activation of the cryostat was reduced with the use of the shielding nose cone, it did not eliminate excess worker dosage. In addition, the copper nose cone itself would become highly activated, and it would have to be replaced after each shot.

Several modifications in the cryostat design are being considered, including the use of a lower-activation stainless steel for structural components, the use of beryllium in a shielding nose cone in place of copper, and the use of beryllium for structural components. Whereas the final design of the cryostat is still several years from completion, it currently appears that the use of beryllium components and shielding is most effective in addressing the dual goals of significantly reducing worker dose and, as in the case of nose cones, allowing the parts to be reusable.

\subsubsection{Waste Reduction Approaches}

\section{Film Processing}

Many of the NIF chamber diagnostics will use cameras to capture data. The use of CCD cameras is planned for most diagnostics, except where equipment would not be expected to survive target chamber conditions. This method significantly reduces the photochemical waste stream associated with traditional photographic methods. The Pollution Prevention Opportunity Assessment_LLNL Site-Wide Assessment of

Photoprocessing Waste Streams discusses in detail the substitution of digital imaging for 
photography in many applications, including laser diagnostics. Often, capital costs of digital photographic equipment can be a barrier to their installation. The program should make every effort to see that this WMin option is implemented where viable.

\subsection{PPOA 6: Debris Shield Decontamination}

Cleaning of debris shields is expected to account for liquid mixed waste. The cleaning plan is not yet final.

\subsubsection{PEIS Item 6: Debris Shield Decontamination WBS 1.8.5.2}

\subsubsection{Process Description}

Debris shields (Figure PPOA 6.1) sit in the FOA and function to protect the optics train of diffractive optics, frequency-conversion crystals, and final focus lenses from target-generated debris. The base material for debris shields incorporates pieces of fused silica glass $\left(\mathrm{SiO}_{2}\right), 43 \mathrm{~cm} \times 43 \mathrm{~cm} \times 1 \mathrm{~cm}$ thick. The base material is covered with a porous glass layer $\left(\mathrm{SiO}_{2}\right.$ with $95 \%$ density of parent material) and an antireflective (AR) sol gel layer.

The debris shields must maintain appropriate transmission characteristics and avoid accumulation of damage from projectiles generated during a shot; hence, the shields are designed for routine removal, cleaning, recoating, and re-installation. Cleaning the debris shields removes tritium as well as gold, copper, aluminum, beryllium, and depleted uranium films and splats. The cleaning method used must be highly effective at removing the metal debris, such that after cleaning, cleanliness as measured by optical transmission exceeds $90 \%$. Tritium must also be removed from the debris shields to a level of 10,000 decays per minute per 100 square centimeters $\left(\mathrm{dpm} / \mathrm{cm}^{2}\right)$ before they can be handled by the optics processing area in Building 391.

The frequent need to clean debris shields makes this process a prime candidate for assessment for waste minimization options. Several methods have been investigated, including $\mathrm{CO}_{2}$ pellet cleaning and acid cleaning. The acid cleaning process is described below. The $\mathrm{CO}_{2}$ pellet cleaning method is discussed in Development of Waste Reduction Options, Section 5.7.1.3 of this PPOA.

At the time of the Title I 65\% design review, the baseline for debris shield decontamination was an acid cleaning process (Figure PPOA 6.2). Because of NIF budget and time priorities, further development of the acid cleaning process has been deferred until the year 2000. The process begins when the debris shields are transferred into secondary containers for transport to a cleaning station located in the target chamber decontamination area. Within the cleaning station, the debris shields are baked under vacuum to remove tritium, then subjected to a high-pressure water rinse. The debris shields move through several heated-water soak tanks. The next step, a dip in $1 \%$ hydrofluoric acid (HF) and 15\% ammonia fluoride, removes the sol gel and porous glass 
layers, then the shields receive a final rinse. After this cleaning process, the debris shields will be tested for radioactivity. If they meet the acceptable $\mathrm{dpm} / \mathrm{cm}^{2}$ guideline, the shields will be sent to the optics processing area for refurbishment. This cleaning system is expected to process 192 debris shields per week.

Supporting Graphics/Misc. Graphics/FOA Assembly

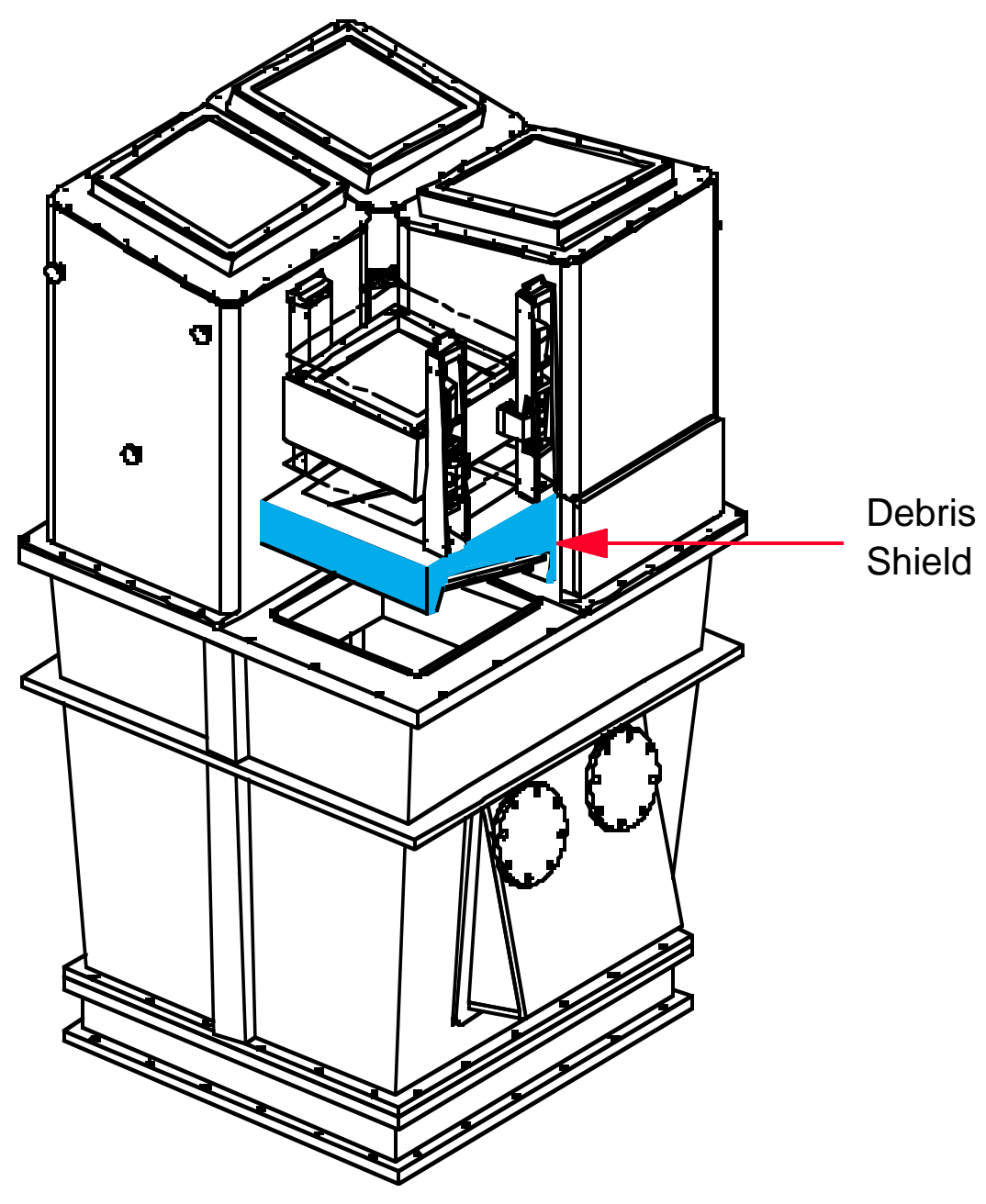

Figure PPOA 6.1. Final optics assembly showing location of the debris shield. Source: T. Reitz, P. Dohoney, F. Drobnik, M. Evans, B. Fischer, S. Holmes, F. Lopez, and Gil Wong. Environmental Protection Systems, WBS 1.8.5 Title I Presentation. November 13, 1996. 


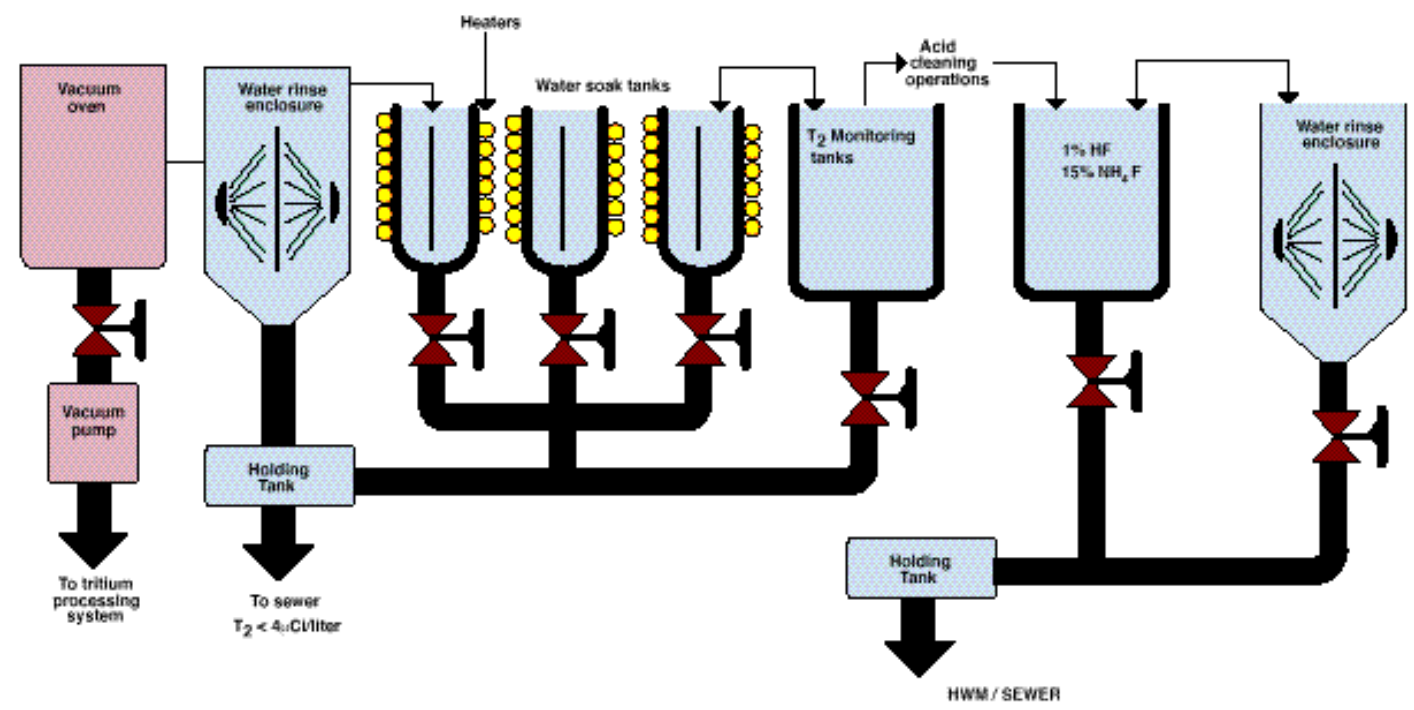

Figure PPOA 6.2. Debris shield decontamination process. Source: T. Reitz, P. Dohoney, F. Drobnik, M. Evans, B. Fischer, S. Holmes, F. Lopez, and Gil Wong. Environmental Protection Systems, WBS 1.8.5 Title I Presentation. November 13, 1996.

\subsubsection{Identification of Waste Stream Components and Amounts}

Tritium from the Vacuum Oven

Tritium baked off in the vacuum oven will be sent to the TPS. Such TPS waste is discussed in PPOA 2.

\section{Rinse Waters and Acid Bath}

It is expected that the rinse water and acid-dip mixture from debris shield decontamination operations will be tritiated. It is estimated that these materials will be sewerable. Current estimates of water $\mathrm{pH}$ and water concentrations of tritium and copper indicate that levels will meet LLNL internal discharge limits shown in Table PPOA 6.1. The estimate of sewerability was based on the conservative assumption that debris shields will be cleaned when there is a uniform $300 \AA$ of deposited material over the entire surface of a debris shield. Rinse water will be tested and characterized prior to disposal. In addition to sewering, other disposal options are currently being considered. The options include onsite or offsite evaporation and containerizing for offsite disposal. The evaporation option has the advantage of volume-reduction. 
Table PPOA 6.1. LLNL internal discharge limits.

\begin{tabular}{|c|c|}
\hline Substance & Sewer discharge limit \\
\hline Tritium & 5.0 ÊCi $/ \mathrm{L}$ \\
\hline Copper & $10 \mathrm{mg} / \mathrm{L}$ \\
\hline Beryllium & $0.74 \mathrm{mg} / \mathrm{L}$ \\
\hline $\mathrm{pH}$ & 5 to 10 \\
\hline
\end{tabular}

Source: C. H. Granfield (1989), Guidelines for Discharges to the Sanitary-Sewer System, Lawrence Livermore National Laboratory, Livermore, CA (UCAR-10235).

Life Span of Debris Shields

Debris shields will be cleaned each week. After several cleanings, it is expected that the shields will require repolishing. Each debris shield will be repolished once or twice. The expected life span is six months. After their useful life span, they will be disposed of, or reused on site for other purposes.

\section{Other Waste Streams}

Waste generated due to personal protective equipment and wipe cleaning is addressed in PPOAs 3, 8A, and 8B.

\subsubsection{Development of Waste Reduction Options}

Investigation of an Option: $\mathrm{CO}_{2}$ Pellet Cleaning

The PSAR indicated that debris shields would be cleaned by $\mathrm{CO}_{2}$ pellet blasting. For some time, this process was the favored alternative to the baseline cleaning process, dipping debris shields in sodium hydroxide solution. $\mathrm{CO}_{2}$ pellet blasting, which would generate minimal waste, was an excellent P2 measure when compared to the baseline process, which would generate a significant quantity of liquid mixed waste.

The use of the $\mathrm{CO}_{2}$ pellet blasting process was abandoned, despite the significant research time and expense invested in it, when it was shown to be ineffective in removing metal splats. Whereas initial tests of the method for cleaning surrogate contaminants (300- $\AA$ gold film) on fused silica proved to be $98 \%$ effective, subsequent tests with Nova-generated shot debris demonstrated only $63 \%$ cleaning efficiency. The higher bond strengths exhibited by the Nova-generated debris were thought to arise from ion stitching. In addition, the aggressive $\mathrm{CO}_{2}$ pellet cleaning parameters needed to remove shot debris were found to create, or even worsen, surface damage on the debris shields. 


\subsubsection{Waste Reduction Approaches}

When, after the year 2000, development of this cleaning process is resumed, the program should continue to evaluate feasible commercially available technologies.

\subsection{PPOA 7: Capacitors, Oil-Filled}

Oil-filled capacitors are expected to generate hazardous wastes from maintenance activities and disposal at the end of their useful life. This PPOA discusses the development of longer-life capacitors for the NIF.

\subsubsection{PEIS Item 7: Capacitors, Oil-Filled WBS 1.3.4.1}

\subsubsection{Process Description}

The main laser components in the NIF take a low-power laser pulse from the master oscillator, shape and smooth it, amplify it enormously, and precisely direct it at a minuscule target. Major laser components include the optical pulse generator, the amplifiers, the pulsed-power system to drive the amplifiers, the optical switch, a final optics assembly to convert frequency to ultraviolet and focus the beams, and the beam control system. Each of these key components has required significant technology advancements. The primary function of the optical pulse-generation (OPG) system, NIF's "front end," is to generate, amplify, and shape the laser beams before they enter the main amplifiers. The OPG, which uses optical fiber technology extensively, was designed to be highly versatile to satisfy the broad range of NIF experimental requirements.

Large amplifier units in each NIF beamline are designed to efficiently amplify the input pulse from the OPG to each of the 192 beams to the required power and energy, maintaining the input beam's spatial, spectral, and temporal characteristics. The amplifiers, with 16 glass slabs per beam, are arranged in two units, the cavity amplifier and the booster amplifier. Together, they form the laser system's central component.

The glass slabs are surrounded by vertical arrays of flashlamps. Measuring nearly $180 \mathrm{~cm}(6 \mathrm{ft})$ of arc length, the NIF flashlamps are the largest commercial units ever made. Each driven with about 30,000 joules of electrical energy, the flashlamps excite the neodymium in the laser slabs to provide optical gain in the infrared spectrum. Some of the energy stored in the neodymium is released as the laser beam passes through.

The NIF power-conditioning system provides energy for the flashlamps with the highest-energy array of capacitors ever built. The system's design is a collaboration among LLNL, Sandia National Laboratories in Albuquerque, NM, and industry. The power-conditioning system will occupy four capacitor bays, each $15 \times 76 \mathrm{~m}(50 \times 250 \mathrm{ft})$, adjacent to each laser cluster. The system must deliver three pulses to each flashlamp: a 
triggering test pulse, a pre-ionization pulse to prepare the lamps for main discharge, and a main pulse to provide energy to the flashlamps.

The latest capacitor technologies will be incorporated, including handling the energy in larger units, and qualifying as many vendors as possible. Very simply, the capacitors are energy-storage devices that use metalized dielectric electrode technology, which includes a dielectric fluid that is used for high-voltage insulation inside the capacitor. Energy is stored in the solid dielectric material, which is either polypropylene or paper. Twenty capacitors will be housed in 1.7-MJ modules that power the flashlamps in parallel. Eight such modules are needed to power each laser bundle of eight beams. The NIF will require approximately 4,000 capacitors, and the capacitor bank will store some 330 megajoules of energy.

The capacitors will use a "self-healing" technology previously tested on Beamlet. The Beamlet is a prototype for one of the 192 beamlines making up the NIF laser. It operates in a regime never before achieved by inertial confinement fusion (ICF) lasers. Figure PPOA 7.1 shows a schematic of a Beamlet capacitor bank module.

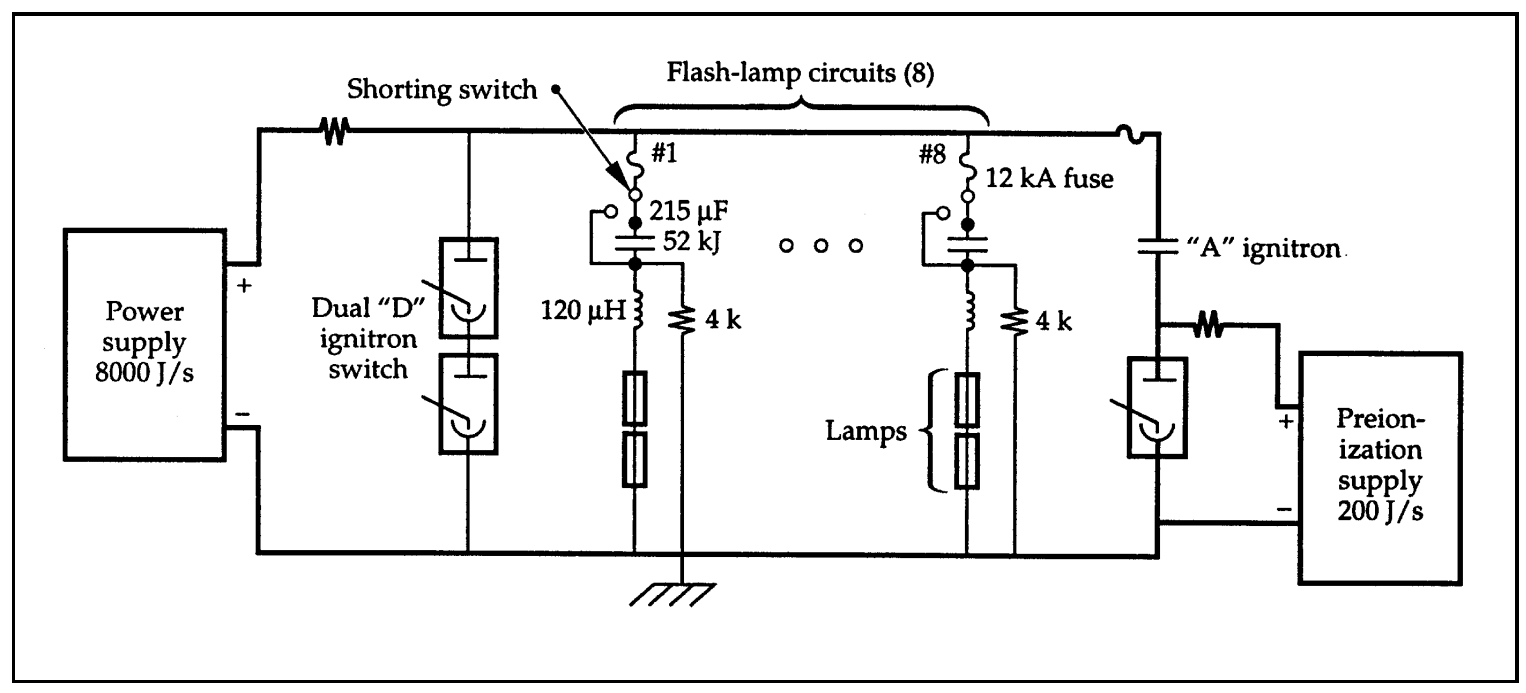

Figure PPOA 7.1. Simplified schematic of a Beamlet capacitor bank module. The same technology will be used for NIF capacitors. Source: D. Larson, Beamlet PulsedPower System (UCRL-LR-105821-95-1).

This technology involves failure characteristics that are more predictable than those of conventional capacitors, resulting in better capacitor bank design at lower cost. This type of capacitor is characterized as self-healing because its "soft" failure mode (small internal faults) causes a gradual loss of capacitance over the capacitor's lifetime. 


\subsubsection{Identification of Waste Stream Components}

The LTAB capacitors will be the dominant source of capacitor waste. The actual capacitor configuration is not yet finalized. Three prototypes will be tested. The third prototype or First Article, will be lifetime-tested, enabling quantitative evaluation of lifetime extensions over previous technologies. The design of power-conditioning module configurations with regard to numbers of capacitors is also still undergoing refinement.

This waste stream will have some capacitor oil in it. The Beamlet capacitors were developed with diethylhexylphthlalate (DEHP) as the dielectric fluid, which is a significant human health hazard. NIF designers are now substituting the DHEP-filled capacitors with less toxic castor-oil or rape-seed-oil-filled capacitors.

\subsubsection{Development of Waste Reduction Options}

The waste stream will be composed of capacitors beyond their useful service life and nonhazardous capacitor oil. The primary mechanism for reducing the volume of this waste stream is the development, or purchase, of capacitors with longer service life than that estimated in the PEIS. Beamlet and NIF designers have driven capacitor design in this direction to a significant extent already, and capacitors used in the Beamlet tests described above have significantly longer service life. They are already expected to meet the 30-year, 20,000-shot design requirement baseline for capacitor lifetime.

Key parameters to note, as capacitors are specified and purchased, are those that allow power-conditioning module configurations to maintain conditions that permit capacitors to operate during the full 30-year lifetime of the facility. Such a service life ensures minimal capacitor waste during the NIF operational lifetime.

No other P2 options are proposed for this waste stream. The best waste minimization option available was to maximize the service life of the units, so that fewer units are retired each year. This has been done through a design effort culminating in Beamlet testing. The next-best option would be to eliminate the use of hazardous dielectric fluids, and, to the extent possible, such elimination is also being implemented.

\subsection{PPOA 8A: General Chemicals}

Operations in the LTAB and $\mathrm{OAB}$ are expected to generate liquid hazardous waste. The P2/WMin Plan addresses methods for minimizing waste streams during various operations, including wastes generated during optics processing (see PPOA 8B, below). Methods for minimizing other general chemical use will be addressed more fully as operations and maintenance procedures are defined. 


\subsubsection{PEIS Item 8A: General Chemical Use}

\subsubsection{Process Description}

Within both the LTAB and $\mathrm{OAB}$, processes or activities performed for the following subsystems are expected to generate wastes that can be part of the general chemicals waste stream:

- Laser systems (WBS 1.3)

- Beam transport systems (WBS 1.4)

- Optical components (WBS 1.6)

- Laser control (WBS 1.7)

- Target experimental system (WBS 1.8)

- Operations special equipment (WBS 1.9)

- Start-up and activation (WBS 1.10)

Other than the Optical Components Processing activities discussed in PPOA 8B, the largest fraction of NIF general chemical wastes will derive from various cleaning activities. Cleaning activities occur in numerous NIF operations. Because a great deal of information is available on reducing the use of cleaning chemicals, Section 7.0, References, includes publications and Web sites that may prove useful.

This PPOA focuses on the cleaning processes that occur during routine operation and maintenance. Pollution prevention associated with the production of KDP crystals is outlined in PPOA 8B.

The NIF design team incorporated P2/WMin technologies into many cleaning processes. If hazardous or environmentally unfriendly products (e.g., solvents) were used in NIF processes, a great deal more chemical waste would be projected. Instead, NIF designers have considered waste generation and minimization at the design stage.

\subsubsection{Chemical Usage in the OAB}

\subsubsection{Process Description}

The OAB will house mechanical parts cleaning facilities for laser subsystems called line replaceable units (LRUs). The type of mechanical parts to be cleaned vary greatly in size, geometry, surface finish, and material. Mechanical parts range in size from machine screw parts to frame-like mechanical structures as long as $2.75 \mathrm{~m}$. Materials will be various grades of aluminum, steel, and stainless steel with different surface finishes. All LRU mechanical parts within the OAB are required to be cleaned to MILSTD-1246C Level 50A or better prior to assembly. Maximum allowable particle sizes and counts are summarized in Tables PPOA 8A.1 and PPOA 8A.2. The sequence of operations for mechanical parts is shown in Figure PPOA 8A.1. 
NIF-0010575

UCRL-AR-131194

Table PPOA 8A.1. MIL-STD-1246C Level 50A: nonvolatile residue (NVR) cleanliness level.

\begin{tabular}{|c|c|}
\hline Level & Limit, NVR (mg/0.1m $\left.{ }^{2}\right)$ \\
\hline A & 1.0 \\
\hline
\end{tabular}

Table PPOA 8A.2. MIL-STD-1246C Level 50A: particle cleanliness level.

\begin{tabular}{|c|c|c|}
\hline Level & Particle size $(\mu \mathrm{m})$ & Count $\mathbf{p e r} \mathbf{1} \mathbf{f t}^{\mathbf{2}}$ \\
\hline 50 & $\geq 5$ & 166 \\
\hline 50 & $\geq 15$ & 25 \\
\hline 50 & $\geq 25$ & 7.3 \\
\hline 50 & $\geq 50$ but $\leq 80$ & 1.0 \\
\hline
\end{tabular}

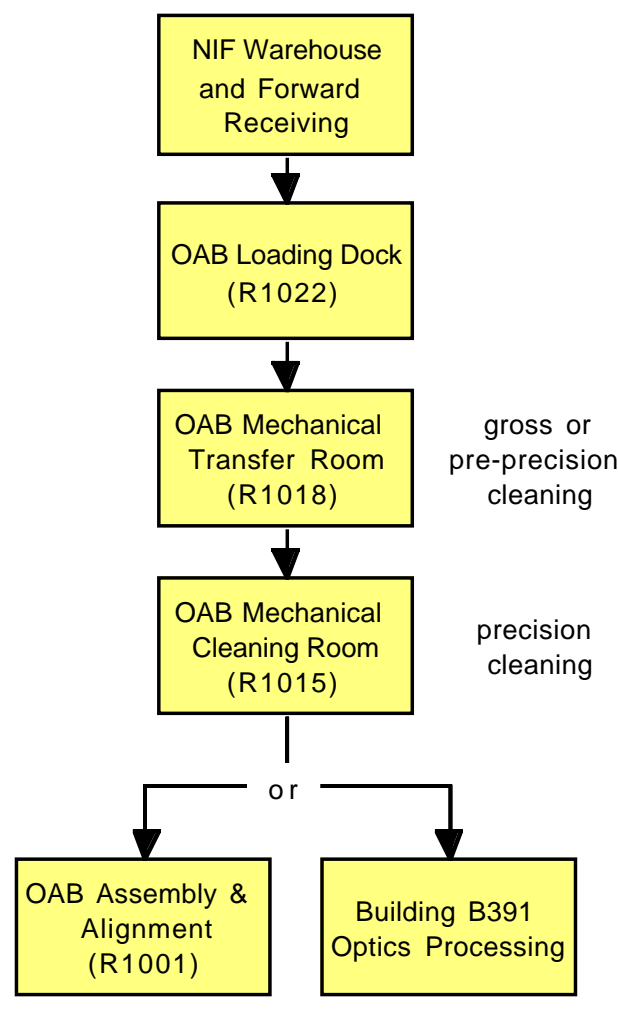

Figure PPOA 8A.1. Optics Assembly Building. Mechanical parts cleaning flow. 
The OAB will have the capability for gross and precision cleaning. Manual wiping, aqueous-based high-pressure spray systems, ultrasonic cleaning systems, and a $\mathrm{CO}_{2}$ snow cleaning system will all be used to clean mechanical parts to achieve the required cleanliness levels.

\subsubsection{Identification of Waste Stream Components}

Contaminants to be removed include dirt, fingerprints and other human soils, weld scab, grit blast debris, packaging and handling contaminants, fabrication process residue, oil, grease, lubricant, cutting fluid, and other machining fluids. At high concentration, some of these contaminants could render waste from cleaning operations hazardous.

\section{Gross Cleaning}

Manual wiping with either water, or 10-20\% isopropyl/ethyl alcohol and water, will be used in some applications to remove surface contaminants. Cloth wipes from the Class 10,000 rooms will be laundered and reused. Kimwipes from the Class 10,000 rooms are expected to be disposed of with the municipal trash. Wipe cleaning with solvents, such as toluene, xylene, and acetone, will be employed in applications where no alternative is suitable, but such cleaning will be minimal. These wipes will be disposed of as solid hazardous waste.

Aqueous-based, high-pressure spray systems will be designed to use water-based chemicals at $71^{\circ} \mathrm{C}$. To conserve energy, these system will recycle the runoff and reuse the solution. Such equipment is commercially available and is currently being prototyped. Specific chemicals to be used are as yet unspecified, but they may include Brulin-type products. Waste generated in this process is expected to be sewerable, depending on its $\mathrm{pH}$ and constituents removed from the surfaces.

\section{Precision Cleaning}

Manual wiping with either water, or 10-20\% isopropyl/ethyl alcohol and water, will be used in some applications to remove surface contaminants to achieve Class 100 level cleanliness. Washable cloth wipes from the Class 100 rooms will be laundered and reused in the Class 10,000 rooms.

Ultrasonic cleaning of small mechanical parts prior to assembly is defined to be a stand-alone, fully enclosed, aqueous-based cleaner. The system consists of several washes, rinses, and drying stations. Waste from this system is expected to be sewerable, depending on its $\mathrm{pH}$ and constituents removed from the surfaces.

A custom-built mechanical parts cleaning system will be used for large parts. Specifications dictate a combination of gross and precision cleaning technologies. The system will use high-pressure spray, as discussed in the gross cleaning section, and ultrasonics with a nonhazardous aqueous solution, as discussed in the precision 
cleaning section. Tanks will each hold approximately $10.2 \mathrm{~m}^{3}$ of ultrasonic cleaning solution. This system will function much like the small-parts washer, and it acts as a backup system for that washer.

$\mathrm{A} \mathrm{CO}_{2}$ snow cleaning system will remove particles from the surface of the object being cleaned through a combination of momentum transfer, dissolution, and thermal shock. Waste from this process will be solid particulate collected on filters or swept up. Characterization of the waste will depend on the nature of the surface cleaned and the materials removed.

\subsubsection{Waste Reduction Approaches}

Many of the large components will be precleaned before they are shipped to the NIF, obviating the need for onsite gross cleaning. The least-hazardous cleaners are being used for wipe operations, in the ultrasonic cleaning system, and in the high-pressure spray system. Brulin-type cleaners have proven effective in the Nova program, and they may be employed in the NIF if they meet cleanliness specifications.

\subsubsection{Chemical Usage in the LTAB}

The LTAB will consist of two laser bays directed at a single target chamber. Each laser bay has two capacitor bays and separate switchyards, with a shared operations support area, diagnostics building, and decontamination area.

The LTAB occupies two main areas. The laser building area houses the laser bays, capacitor bays, periscope structure, mechanical and computer controls, and two of the switchyards. The target bay chamber area holds the target bay and the two remaining switchyards. Both are considered Class 100,000 clean rooms. However, some of the systems within these areas have "cleaner" requirements. The maximum allowable particle sizes and counts are summarized in Table PPOA 8A.3.

Table PPOA 8A.3. MIL-STD-1246C Level 100A (internal optics cavity) and 500 (external structures) particle cleanliness levels.

\begin{tabular}{|c|c|c|}
\hline Level & Particle size $(\mu \mathbf{m})$ & Count per $\mathbf{1} \mathbf{f t}^{\mathbf{2}}$ \\
\hline \multirow{2}{*}{100} & $\geq 5$ & 1785 \\
& $\geq 15$ & 265 \\
& $\geq 25$ & 78 \\
& $\geq 50$ & 11 \\
& $\geq 100$ & 1 \\
\hline 500 & $\geq 50$ & 11817 \\
& $\geq 100$ & 1100 \\
& $\geq 250$ & 26 \\
& $\geq 500$ & 1 \\
\hline
\end{tabular}




\subsubsection{Process Description}

In the $\mathrm{LTAB}$ as in the $\mathrm{OAB}$, the types of mechanical parts to be cleaned vary greatly in size, geometry, surface finish, and material. The LRUs may have to be cleaned in place occasionally, as will surfaces such as panels and walls.

\subsubsection{Identification of Waste Stream Components}

Contaminants to be removed from the line-replaceable units (LRUs) include residues from prior cleaning processes, laser-induced photoablation of surfaces, flashlamp-induced photodegradation of surfaces, airborne materials, fingerprints, and other human soils. Metals and deposits (splats) must be removed from the debris shields and chamber walls. At high concentrations, some of these contaminants could render any waste from cleaning operations hazardous, mixed, or radioactive.

\section{Gross Cleaning}

Manual wiping with either water, or 10-20\% isopropyl alcohol and water, will be used in some applications for the beam transport systems, such as the switchyard, laser bay, spaceframe, and laser bay concrete contaminants. This will ensure $\leq$ Level 500 cleanliness.

Manual wiping with either water, or 10-20\% isopropyl alcohol and water, will be used in some applications to remove surface contaminants from vacuum beam tubes, deformable mirrors, and the periscope structure. This will ensure $\leq$ Level 100 cleanliness.

\section{Precision Cleaning}

Aqueous-based, high-pressure water spray systems will be designed to use waterbased chemicals at $71^{\circ} \mathrm{C}$ for the beam tubes, which are approximately $6 \mathrm{~m}$ long. The vacuum tubes within the beam transport system require Level 100A cleanliness, in accordance with MIL-STD 1246C. Vacuum tubes will be cleaned using the aqueousbased, high-pressure water spray. To conserve energy, the system will recycle runoff and reuse the solution. This equipment is not commercially available and is under design. Specific chemicals to be used are as-yet unspecified, but they may include Brulin-type products. Waste generated in this process is expected to be sewerable, depending on its $\mathrm{pH}$ and constituents removed from the surfaces.

The $\mathrm{CO}_{2}$ snow cleaning system will remove particles from the surface of the object being cleaned through a combination of momentum transfer, dissolution, and thermal shock. Waste from this process will be solid particulate collected on filters or swept up. Characterization of the waste will depend on the nature of the surface cleaned and the materials removed. Beam tubes may be cleaned with a $\mathrm{CO}_{2}$ snow cleaning system if it meets cleaning specifications. 
Designers for both OAB and LTAB activities have actively investigated P2 options in all the designs of the facility. Nonhazardous or less-hazardous products are being employed where possible. Hazardous solvents have been nearly eliminated, and the use and reuse of washable wipes has been planned.

\subsubsection{Development of Waste Reduction Options}

Megasonic Cleaning

Megasonic cleaning, sometimes called acoustic streaming, uses high-frequency acoustic waves to generate pressure waves in a fluid. The fluid can vary from water to alkalines, acids, or alcohols.

Megasonic cleaning is used primarily for particle removal. Particles as small as $0.3 \mu \mathrm{m}$ can be removed with this method. Only the portion of the material facing the transducer will be cleaned. Typical exposure time is 10 to 30 minutes, and effectiveness increases with temperature. Megasonic cleaning is also used to enhance the efficiency of detergent and surfactant cleaners.

Unlike ultrasonic cleaning that uses 20- to $40-\mathrm{kHz}$ frequencies, megasonics uses 700to $1000-\mathrm{kHz}$ frequencies. Parts that cannot be cleaned with ultrasonics because of cavitation effects, may be candidates for megasonic cleaning.

\section{Supercritical Fluid Cleaning}

Supercritical fluid cleaning involves high-pressure fluid that penetrates small openings. It is especially effective as a precision cleaning system for liquid contaminants. $\mathrm{CO}_{2}$ is the most widely publicized fluid used in supercritical cleaning applications.

The advantages of a supercritical cleaning system include the ability to clean intricate components (gyroscopes, nuclear valve seals, and porous ceramics); the ability to clean liquid contaminants (adhesive and flux residues, dielectric oils, and some fats and waxes); a high degree of cleanliness; relatively short cleaning times; and low operating costs.

The disadvantages are the inherent safety conditions that must be addressed because of high pressure and high temperature, large capital costs, constraints on component size (limited by the vessel in which a component is immersed), and poor removal of hydrophilic contaminants.

\section{Hand Wipe Alternative}

The use of a packaged hand wipe system, such as the Wet Task Wiping System used by the U.S. Navy, reduces the amount of cleaner consumed. The wipes are 
premoistened and stored in a closed container. Many automotive factories use a similar packaged hand wipe system

The user pulls a premoistened wipe through a small opening in the container. The packaged hand wipe system can be custom ordered. Containers are available in a variety of sizes, and user-specified cleaners can be added to the containers.

The advantages of this system include a reduction in air emissions because the wipes and cleaners are in a closed container, and a reduction in wasted excess cleaner while wetting the wipes. In addition, several vendors currently offer such products.

One disadvantage at present is that the system uses one-time-use wipes, which are disposed rather than recycled.

\subsection{PPOA 8B: General Chemicals, Optical Component Processing}

This operation involving general chemicals is expected to generate liquid hazardous waste. The P2/WMin plan addresses methods for minimizing waste streams during various operations, including wastes generated during optics processing.

\subsubsection{PEIS Item 8: General Chemicals Optical Component Processing in B391 WBS 1.6.8}

\subsubsection{Process Description}

The optics processing facility, located in Building 391 (B391), will handle the largeaperture optics installed at the NIF. Such optics must be precision cleaned prior to coating and installation. If contaminant particles remain on an optic, when it is inserted into the beamline, the particles will absorb flashlamp or laser light and explosively vaporize, resulting in serious damage to the polished and coated surfaces. The B391 facility will clean optics, apply AR coatings, inspect for cleanliness, and transport them on to the OAB. Additional space in B392 will be used to clean and coat small (15- to 20$\mathrm{cm})$ optics.

\section{Arrival of Optics}

NIF optics shipped from vendors will arrive at LLNL main receiving and will be transferred to the NIF optics storage facility. They will be inspected, logged into a quality assurance system, and placed in storage until needed according to the NIF production schedule. When ordered from storage, optics will enter the B391 optics processing facility through an airlock into the Class 10,000 transfer area. B391 will house two Class 100 clean room areas: one for processing laser slabs, mirrors, lenses, windows and debris shields; another for processing potassium dihydrogen phosphate (KDP) crystals or their deuterated analog $\left(\mathrm{KD}^{*} \mathrm{P}\right)$. 
Optics arriving at the airlock will be encased in several layers of packaging and bagging. The innermost layer is a PET-G container, which is a tough, wipeable, recyclable plastic. The outer two layers of bagging will be removed in the airlock. The final layer will be removed in the transfer area. The PET-G container will be wiped, and the optic will be removed and transported into the Class 100 optics processing area. There, it will be inserted into a reusable support fixture in preparation for cleaning. KDP crystals will be delivered from the transfer area to the KDP processing area. All bagging material will be recycled.

\section{Aqueous Cleaning Lines}

Inside the optics processing area, each glass optic will be placed into one of three cleaning lines. Two automated, aqueous cleaning lines will be physically identical and easily programmed to handle different types of optics, such as fused silica or amplifier slabs. The third cleaning line will be configured for cleaning and coating blast shields. All optics cleaning equipment will be clean-room-compatible, designed to work with minimal lubrication, and constructed of materials that minimize particle generation.

The aqueous precision cleaning lines for fused silica, amplifier slabs, mirrors, and polarizers will include the following features:

- Manual scrub stations.

- Ultrasonic immersion tanks for precision cleaning.

- 18-M $\Omega$, deionized-water sprays and ultrasonic immersion tanks for removal of particulates and wash solutions.

- Drying station using one or more of the following methods - Slow pull out of a heated bath, or - Air drying at room temperature.

The approximate dimensions of the tanks will be $90 \mathrm{~cm}$ long $\times 60 \mathrm{~cm}$ wide $\times 20 \mathrm{~cm}$ deep. The cleaning lines will handle various sized optics up to $80 \times 50 \times 10 \mathrm{~cm}$. These cleaning lines will be programmable and can be configured for different optics.

For the more robust optics made from fused silica, or dielectric-coated BK-7 mirrors and polarizers, the baths will be heated and will contain neutral to strong alkaline cleaning agents. For more easily damaged optics, such as dielectric coated mirrors and polarizers, the ultrasonic frequency will be modified to prevent damage.

For cleaning of the most delicate glass optics, the amplifier slabs, the cleaning line will be configured with suitable thermal, mechanical, and chemical parameters. Baths will be cooled to maintain an operating temperature no higher than $7^{\circ} \mathrm{C}$ above room temperature. Neutral cleaning agents and high-frequency ultrasonics will be used. These optics will dry at room temperature. After cleaning, fused-silica optics will enter a sol gel dip-coating system. 
The third and separate aqueous cleaning line will be devoted solely to blast shields. This line is expected to be partially automated and capable of processing 10 blast shields per day. Blast shields brought to the optics processing area will be put into cleaning frames. The expected cleaning sequence will include a deionized water rinse, a wash with a surfactant solution, a polishing step, and a light citric acid wash. This may be followed by a final deionized water rinse. After cleaning, blast shields will be sol gel coated using a meniscus-coating method.

\section{Sol Gel Production and Coating}

Colloidal silica sol gel is a suspension of 10- to 30-nm colloidal in alcohol, which dries on the optic and produces a coherent quarter-wave AR coating. Colloidal silica sol gel will be prepared on-site by mixing purified tetraethyl orthosilicate (TEOS) with anhydrous ethanol and an ammonium hydroxide catalyst solution. Small quantities of other sol gel coating solutions will be prepared from either purchased silicone resin or silicate and silicone-silicate solutions prepared in-house by reaction of TEOS and methyl triethloxy silane (TMOS). These solutions will be used for blast shield and KDP coating layers.

Following cleaning, all of the fused silica optics will receive a colloidal sol gel, AR coating. Sol gel dip tanks are enclosed and have a nitrogen environment while the tank is closed. The nitrogen and ethanol is exhausted before the system enclosure is opened. The sol gel systems will be automated with the ability to handle different sized optics up to about $80 \mathrm{~cm}$. The optics will be transferred manually to the sol gel tank and then to the assembly area for installation into optical mounts.

After cleaning, blast shields will be AR coated using a meniscus coating method. In this method, a bar extruding a bead of sol gel will be drawn across the blast shield, leaving a thin layer of the sol gel solution that dries to produce the final coating. To provide the optimal broadband performance, the blast shields will be coated with a two-layer coating. The first layer will be a silicate; the second layer will be a colloidal silica. To make the colloidal silica coating more scratch- and abrasion-resistant, the coated blast shields will be hardened by placing them in an enclosure with a container holding a small volume of ammonium hydroxide for approximately 12 hours. After hardening, the blast shields will be transferred to a workstation where they will be sealed into their final frames. The blast shields will be transported out of B391 in an enclosed cart in a fashion similar to that for other optics.

\section{Cleanliness Monitoring}

Following cleaning and coating, all large-aperture precision optics will go to a cleanliness verification station for visual or automated inspection. Optics are expected to achieve Level 20A cleanliness under MIL-Standard 1246C. Other optics performance measurements that may also be performed at this time include photometry of coated optics and scratch-dig inspection of bare optics. 
Upon passing inspection, optics will be transferred to the Class 100 packaging area, loaded onto a clean transport cart, sealed, and backfilled with nitrogen gas. The cart will be double-bagged, then will leave $\mathrm{B} 391$ for transport to the OAB, where each optic will be removed from the clean cart and transferred into the appropriate assembly area.

\section{KDP Processing Area}

A separate clean room is necessary for cleaning, coating, and processing KDP and KD*P crystals because accidental exposure to ammonia vapors can cause reaction with and fogging of the crystals. The automated, fully enclosed, KDP-crystal cleaning system will process KDP optics (up to $41 \times 41 \mathrm{~cm}$ ) through a series of solvent sprays, soaks, and ultrasonic immersion steps. The process will finish with a controlled solventevaporation step. Toluene is the solvent of choice for cleaning KDP crystals. Secondary butanol (sec-butanol) or another polar solvent will also be used.

All crystals will be spin coated with de-ammoniated, colloidal silica sol gel. Spin coating, as opposed to dip coating, creates a negligible liquid waste stream, and it represents a significant $\mathrm{P} 2$ technology when compared to dip coating. The conversion crystals may be silicone or silicone-silicate coated prior to sol gel coating. After coating, the crystals will be inspected, installed in their optical mount, packaged, and shipped to the $\mathrm{OAB}$ in a fashion similar to that for other optics.

\subsubsection{Identification of Waste Stream Components}

\section{Optics Packaging}

Much of the optics packaging will be reused. Shipping containers in which NIF optics arrive from vendors will be sent back for reuse, as will the PET-G containers. The layers of nylon and polyethylene bagging will be recycled.

\section{Aqueous Cleaning Lines}

The chemicals that will be used in the aqueous cleaning lines are as yet unspecified. One possibility is the use of a nonhazardous Brulin-type product containing a surfactant. When changed out, it is highly likely that the tank contents will be sewerable.

\section{Waste Sol Gel}

The useful life of sol gel will be approximately six months. After that time, approximately $150 \mathrm{~L}$ of sol gel will be disposed of as hazardous waste. There will be two tanks in the processing area. 
Waste Silicone, Silicone-Silicate, and Silicate Sol for KDP and Blast Shields

Although this coating could generate waste silicone solution, it will be of relatively small volume because it is applied either by meniscus or spin coating.

\section{KDP Solvent Tanks}

Designers have used a performance specification method to ensure that low solvent usage is designed into all bids for KDP cleaning-system equipment. In the specification, the total solvent volume associated with the system will not be more than $273 \mathrm{~L}$. The system is expected to clean 800 optics in three years. The targeted maximum quantity of clean solvent to be used for each optic is $\sim 8 \mathrm{~L}$.

There is little information with which to estimate the quantity of solvents that will be used following the production phase for KDP optics. Toluene used in these processes may have sufficient purity, when retired, for reuse at other LLNL locations without treatment.

\section{Personal Protective Equipment and Wipes}

Gowns and wipes will be associated with both normal clean room operation and maintenance. Clean room gowns will be laundered by an off-site vendor and reused. Wipes from the Class 100 rooms will be laundered and reused in the Class 10,000 area.

\subsubsection{Development of Waste Reduction Options}

\section{Optics Packaging}

The nylon and polyethylene bagging protecting the cleanliness of crated optics will be recycled off site. Although it is unlikely that this material can be reused on site, efforts should be made to maximize off-site recycling of this packaging.

\section{Sol Gel Recycling}

Sol gel recycling could be used to recover ethanol, potentially recapturing up to $80 \%$ of the waste stream volume. Work at Nova has proved that such recycling is possible. Although no location is planned for this process at the NIF, it may be possible to establish a central sol-gel-recycling operation for use by both the NIF and the LLNL Atomic Vapor Laser Isotope Separation (AVLIS) Program.

\section{Solvents from KDP Processing}

Even though waste solvents from the KDP cleaning system are unfit for continued use with KDP, they will still be relatively clean. Their contaminants are expected to be 
organics (primarily a mineral oil) and particulates. It is appropriate to define the levels of contamination and then seek reuse applications for these solvents on site. See, for example, the discussion of KDP solvent tanks, above.

\subsubsection{Waste Reduction Approaches}

Several processes associated with optics processing have been eliminated or modified, resulting in the significant reduction or elimination of waste streams.

\section{Sol Gel Coating}

Two examples of design changes in application methods for sol gel will result in the generation of significantly less sol gel waste. These changes include:

- Transition from dip coating to spin coating for KDP crystals.

- Design of a meniscus coater for blast shields to replace dip coating.

Identical Optics Processing Aqueous Cleaning Lines

Identical cleaning lines build redundancy into the cleaning system. Such redundancy will prevent the need to switch to hand wiping using ethanol drying in the event of failure of one of the lines.

\section{TEOS Purification}

For past operations, such as those at the Nova laser, purified TEOS for sol gel has been prepared on-site by refluxing purchased low-purity ethylsilicate to remove ethanol and other byproducts. For NIF operations, a high-quality source for purchasing TEOS has been identified, eliminating the need for the purification step and its resultant waste stream, which is approximately $50 \%$ of the raw feed stream.

\section{Sol Gel De-Ammoniation for KDP}

Sol gel for use with KDP crystals will be purchased. This approach eliminates the need to create equipment to de-ammoniate sol gel on the premises and its resulting waste stream.

\section{KDP Cleaning System Equipment Specification}

The "KDP Cleaning System Equipment Specification Language" is effective in proactively including P2/WMin strategies in the design of the system. In addition to specifying quantitative goals for the amount of solvent used per optic, the specification presents design strategies to achieve them. Examples of options to minimize solvent use include: 
NIF-0010575

UCRL-AR-131194

- Filtering solvent while in use to reduce the need for clean solvent.

- Incorporation of a countercurrent strategy that draws from reservoirs of working solvent of intermediate cleanliness for initial cleaning.

- Multiple strategies for minimizing solvent evaporation. 


\subsection{Accomplishments to Date}

As the NIF proceeds from design and construction, through start-up, and then to operational status, major actions have been taken and will be taken to minimize potential waste generation. Some of the P2/WMin accomplishments are already documented in the predictive PPOAs in Section 5.0 of this document. Some have occurred in other areas of design.

Table 6.1 is a brief running list of the P2/WMin accomplishments for the NIF through June 1998. As of the publication date of this NIF Pollution Prevention and Waste Minimization Plan, only the design and construction phase accomplishments are noted. Table 6.1 will be updated annually as the NIF moves into routine operations, and it will remain a part of the P2/WMin plan as a means of reminding all NIF personnel that their efforts in these areas are important. 
Table 6.1. Pollution prevention and waste minimization accomplishments to date for the NIF.

\begin{tabular}{|c|c|c|c|}
\hline $\begin{array}{l}\text { Description of } \\
\text { minimized waste } \\
\text { stream }\end{array}$ & Source of waste & Waste type & Description of $\mathrm{P} 2$ accomplishment \\
\hline Activated concrete & D\&D activities & Solid LLW & $\begin{array}{l}\text { Developed concrete formulations (in } \\
\text { design phase of facility) with low } \\
\text { activation characteristics to minimize } \\
\text { activation during facility lifetime }\end{array}$ \\
\hline Oily waste & $\begin{array}{c}\text { Vacuum pump } \\
\text { operations and } \\
\text { maintenance }\end{array}$ & Mixed & $\begin{array}{l}\text { Researched vacuum pumps, and } \\
\text { changed design so that oil-free } \\
\text { pumps can be used }\end{array}$ \\
\hline $\begin{array}{l}\text { Aqueous solvent } \\
\text { waste }\end{array}$ & Parts washing & Hazardous & $\begin{array}{l}\text { Developed specification for parts } \\
\text { washers that disallows the use of } \\
\text { solvents or other hazardous materials }\end{array}$ \\
\hline $\begin{array}{l}\text { Solid, hazardous } \\
\text { waste }\end{array}$ & $\begin{array}{l}\text { Capacitor maintenance } \\
\text { and changeout }\end{array}$ & Hazardous & $\begin{array}{l}\text { Designed and tested capacitors that } \\
\text { have significantly longer service life } \\
\text { (than Nova), thus reducing the } \\
\text { number of capacitor changeouts per } \\
\text { year }\end{array}$ \\
\hline Soil, concrete & NIF construction & $\begin{array}{l}\text { Nonhazardous } \\
\text { solid }\end{array}$ & $\begin{array}{l}\text { To the extent possible, soil from NIF } \\
\text { construction is reused onsite, saving } \\
\text { LLNL the cost of offsite transport. } \\
\text { Concrete and soil sent to landfill may } \\
\text { be used as road base and cover. }\end{array}$ \\
\hline Wood and metal & NIF construction & $\begin{array}{l}\text { Nonhazardous } \\
\text { construction } \\
\text { debris }\end{array}$ & $\begin{array}{l}\text { Wood and metal construction debris } \\
\text { recycling programs have been set up } \\
\text { at the NIF construction site, with bins } \\
\text { for each and routine collection } \\
\text { schedules }\end{array}$ \\
\hline Mercury & $\begin{array}{l}\text { Switch failures and } \\
\text { maintenance }\end{array}$ & $\begin{array}{c}\text { RCRA } \\
\text { hazardous }\end{array}$ & $\begin{array}{l}\text { Adapted and tested switches without } \\
\text { mercury components for the power } \\
\text { conditioning system }\end{array}$ \\
\hline Hardware and PPE & $\begin{array}{l}\text { Target positioner and } \\
\text { PPE associated with } \\
\text { cleaning it }\end{array}$ & Solid LLW & $\begin{array}{l}\text { Cryogenic target positioners will } \\
\text { become activated during high-yield } \\
\text { experiments. A nose-cone concept } \\
\text { was developed to attenuate and } \\
\text { scatter neutrons. Nose-cone materials } \\
\text { have been studied to increase service } \\
\text { life and reduce thickness, thus } \\
\text { minimizing LLW. }\end{array}$ \\
\hline
\end{tabular}




\subsection{References}

\section{LLNL Documents}

Bergman, W. (1996), Requirements for a Cleanable Steel HEPA Filter Derived from a Systems Analysis, Lawrence Livermore National Laboratory, Livermore, CA, UCRL-ID-125048 (June, 1996).

Cantwell, B. (1998), "DfE for the NIF," DOE Pollution Prevention Conference XIV, Seattle, WA, June 1-4, 1998.

Celeste, J. (1995), Reassessment of LLNL Waste Generation for Calendar Year 1995, Lawrence Livermore National Laboratory, Livermore, CA, UCRL-AR-125047 (1995).

Gabor, K. (1998), Carbon Dioxide Snow Cleaning Cost Analysis Report, presented to the Pollution Prevention Group (January 20, 1998).

Granfield, C. H. (1989), Guidelines for Discharges to the Sanitary-Sewer System, Lawrence Livermore National Laboratory, Livermore, CA, UCAR-10235 (1989).

Latkowski, J. and J. Sanchez, (in press) Neutronics and Activation Analysis for the National Ignition Facility Cryogenic Target Positioner, Lawrence Livermore National Laboratory, Livermore, CA (June 1998).

Lawrence Livermore National Laboratory (1996), Chemical Inventory Needs of the National Ignition Facility, Lawrence Livermore National Laboratory, Livermore, CA, NIF 0000467 (September 1996). Attachments.

Lawrence Livermore National Laboratory (1994), National Ignition Facility Conceptual Design Report, Lawrence Livermore National Laboratory, Livermore, CA, UCRLPROP-117903, 3: Conceptual Design, NIF-LLNL-94-113 (May 1994) L-16973-1.

Lawrence Livermore National Laboratory (1998), LLNL Mechanical Engineering Specification, Lawrence Livermore National Laboratory, Livermore, CA (March 1998).

MEL98-002-OA: Cleaning of Cast Aluminum Alloy Components for NIF. MEL98-003-OA: Cleaning of Wrought Aluminum Alloy Components for NIF. MEL98-004-OA: Cleaning of 300/400 Series Stainless Steel Components for NIF. MEL98-005-OA :Cleaning of Copper and Copper Alloy Components for NIF. MEL98-011-OA: Gross Cleaning of NIF Components using High-Pressure Water Wash.

Lawrence Livermore National Laboratory (1997), National Ignition Facility Decontamination and Decommissioning Plan, Lawrence Livermore National Laboratory, Livermore, CA, NIF-LLNL-97-0001670 (February 1997), L-19754-1, NIF WBS 1.1.3. Attachments.

Lawrence Livermore National Laboratory (1996), National Ignition Facility Preliminary Safety Analysis Report, Lawrence Livermore National Laboratory, Livermore, CA, UCRL-ID-123759, NIF-LLNL-96-238 (September 30, 1996) L-21729-1. 
Lawrence Livermore National Laboratory, Pollution Prevention Plan, Lawrence Livermore National Laboratory, Livermore, CA, UCRL-AR-127073 (refer to current annual edition).

Lawrence Livermore National Laboratory (1997), Storm Water Pollution Prevention Plan, Lawrence Livermore National Laboratory, Livermore, CA (refer to current annual edition).

Pacific Northwest Laboratory (1996), HEPA Filter Disposal Checklist, Pacific Northwest Laboratory, Portland, OR (October 1996). Contact the current 300 Area Balance of Plant Facility Services Representative.

Parsons Infrastructure and Technology Group (1997), National Ignition Facility Laser $\mathcal{E}$ Target Area Building Conventional Facilities Energy Conservation Report, NIF-0004309 (December 1997), WBS 1.2.1.

Pollution Prevention Group (1996), Draft-NIF Hazardous Waste Management Plan, presented to NIF management for review October 1996.

United States Department of Energy (1996), Final Programmatic Environmental Impact Statement for Stockpile Stewardship and Management, III, Appendix I, Office of Defense Programs, Oakland Operations Office, Oakland, CA, DOE/EIS-0236 (November 1996).

United States Department of Energy (1997), National Ignition Facility Mitigation Action Plan, Department of Energy, Office of Defense Programs, Oakland Operations Office, Oakland, CA, NIF-0001726 (February 1997) WBS No. 1.11.3.

United States Department of Energy (1996), Record of Decision: Programmatic Environmental Impact Statement for Stockpile Stewardship and Management, Office of Defense Programs, Oakland Operations Office, Oakland, CA, DOE/6450-01-P (December 18, 1996). Attachments.

Wong, H. (1998), NIF Optics Assembly Building Small Mechanical Parts Precision Cleaner Equipment Specification, Lawrence Livermore National Laboratory, Livermore, CA, NIF-0005762, REV OA (March 1998).

Wong, H. (1998), "Small Mechanical Parts Precision Cleaner," NIF presentation NIF0005753-OA (March 16, 1998), WBS 1.9.3.1.

\section{Other References, Including Internet Sites}

Joint Service Pollution Prevention Opportunity Handbook

http:/ / enviro.nsesc.navy.mil/p2library (includes for each alternative, sections entitled: overview, material compatibility, safety \& heath, point of contacts, economic analyses, and vendors).

Environmental Site Hot Lists: http:/ /cpas.mtu.edu/hotlist/

SAGE: Solvent Alternatives Guide, developed by the Surface Cleaning Program at

Research Triangle Institute in cooperation with the US EPA Air Pollution

Prevention and Control Division, http:/ /clean.rti.org/

EPIC: United States Department of Energy Pollution Prevention Information Clearinghouse, http:/ /epic.er.doe.gov/epic/ (login required). 


\section{Appendix A Method of Preparing a PPOA}

To complete a PPOA, the NIF will follow the procedure outlined in the DOE performance-based training course, "Pollution Prevention Opportunity Assessment." Any process under study for P2/WMin will be reviewed several times.

The methodology for analyzing a process is based on the Total Quality Method (TQM) problem-solving model. In brief, the TQM involves:

- Evaluating the process input materials and parameters.

- Identifying the pollution waste streams.

- Generating and evaluating options for pollution prevention.

The TQM approach also uses a graded technique that allows investigators to develop PPOAs to varying levels of detail. The toxicity, cost, and volume of a process waste stream directly determine the level of detail required in a PPOA, and that detail is represented as Levels I, II, or III, as discussed below.

\section{Development of Waste Reduction Options}

After developing a detailed understanding of a waste-generating process, the assessment team develops various technical, procedural, and administrative options that would reduce waste. These options are evaluated for estimated cost, technical and economic feasibility, anticipated savings, ROI, and anticipated reduction of a specific waste stream. This option summary is followed by the final PPOA recommendations, which typically include a preferred course of action.

Timetables for implementing the selected option, or combination of options, for each PPOA are provided. Implementation periods vary widely, and they are often highly dependent on funding.

\section{Source-Reduction Approaches}

Investigators evaluate the following types of changes and improvements to generate source-reduction options:

- Material input changes.

- Operational improvements.

- Production process changes.

- Product reformulation. 
- Administrative changes.

Source-reduction alternatives are identified in PPOA team brainstorming meetings. Later, the team leader investigates the alternatives by:

- Searching the literature and vendor catalogs.

- Attending conferences, seminars, and workshops.

- Acquiring baseline information from

- On-line databases such as EPIC, the Environmental Protection Agency (EPA) Tech Transfer Network, EPA's Enviro\$ense, TIE, P2 TECH, Eco Net, EnviroWeb, GNET, and Teltech, and

- Other DOE agencies, including Lawrence Berkeley Laboratory, Sandia National Laboratories, Los Alamos National Laboratory, and Idaho National Engineering and Environmental Laboratory.

- Attending industrial outreach symposiums or technical exchange meetings.

\section{Level I and II Assessments}

If the process is relatively simple (Level I or II), a P2 engineer evaluates the options using standard engineering principles and criteria, such as cost avoidance, payback, waste volume reduction, estimated process lifetime, feasibility, regulatory compliance, implementation period, improved operation/product, and environment, safety, and health impacts. Simple options requiring little or no cost are implemented immediately when applicable.

\section{Level III Assessments}

More complicated assessments (Level III) are evaluated with a weighted sums option table where numerical values (weights) are assigned to the previously mentioned criteria. A scale factor is developed for the associated option, the weight and scale factor products are summed, and the options are compared for the highest score.

\section{Technical Feasibility of Options}

The technical feasibility of an alternative is determined by a P2 or programmatic engineer who uses standard engineering principles and whose primary goal is to maintain process or product reliability and efficiency. The technical success of a P2 option is evaluated using the Weighted Sums Option Evaluation Form described above. Options are scaled according to three categories:

1. No major technical breakthroughs are required, well-designed plans exist to meet the objectives, and the option has a successful track record.

2. Technical advancement is needed; key issues are identified, but no contingency plans exist. 
3. Major technical breakthroughs are required, but lacking. Adequate plans for meeting objectives or key problems are not identified.

\section{Economic Feasibility of Options}

The economic feasibility of an option is determined by evaluating its implementation costs, incremental operating costs, and incremental intangible costs, such as liability or fines. A simple ROI is calculated using the DOE methodology described as follows:

\section{Return on Investment Calculation}

$$
\mathrm{ROI}=\frac{(\mathrm{B}-\mathrm{A})-[(\mathrm{C}+\mathrm{E}) / \mathrm{L}]}{(\mathrm{C}+\mathrm{E})} \times 100
$$

Useful project life (years) $=$ (L)

Operating and maintenance annual recurring costs (annual operating costs)

Annual costs, before implementing the option = Annual costs, after implementing the option = Net annual savings

Project funding requirements (one-time implementation costs)

Capital investment Operating expense Total project funds (B) $-(\mathrm{A})$
$=$ 